\title{
Distribution of values of quadratic forms at integral points
}

\author{
P. Buterus ${ }^{1}$ • F. Götze ${ }^{2}$ - T. Hille ${ }^{3}$ • G. Margulis ${ }^{4}$
}

Received: 9 October 2019 / Accepted: 16 September 2021 /

Published online: 31 January 2022

(C) The Author(s) 2022

\begin{abstract}
The number of lattice points in $d$-dimensional hyperbolic or elliptic shells $\{m: a<Q[m]<b\}$, which are restricted to rescaled and growing domains $r \Omega$, is approximated by the volume. An effective error bound of order $o\left(r^{d-2}\right)$ for this approximation is proved based on Diophantine approximation properties of the quadratic form $Q$. These results allow to show effective variants of previous non-effective results in the quantitative Oppenheim problem and extend known effective results in dimension $d \geq 9$ to dimension $d \geq 5$. They apply to wide shells when $b-a$ is growing with $r$ and to positive definite forms $Q$. For indefinite forms they provide explicit bounds (depending on the signature or Diophantine properties of $Q$ ) for the size of non-zero integral
\end{abstract}

Research supported by the DFG, CRC 701.

$凶$ F. Götze

goetze@math.uni-bielefeld.de

P. Buterus

buterus@mathematik.uni-goettingen.de

T. Hille

thomas.hille@northwestern.edu

G. Margulis

grigorii.margulis@yale.edu

1 Department of Mathematics, University of Göttingen, Göttingen, Germany

2 Faculty of Mathematics, University of Bielefeld, Bielefeld, Germany

3 Department of Mathematics, Northwestern University, Evanston, IL, USA

4 Department of Mathematics, Yale University, New Haven, CT, USA 
points $m$ in dimension $d \geq 5$ solving the Diophantine inequality $|Q[m]|<\varepsilon$ and provide error bounds comparable with those for positive forms up to powers of $\log r$.

Mathematics Subject Classification 11P21 $\cdot$ 11D75

\section{Contents}

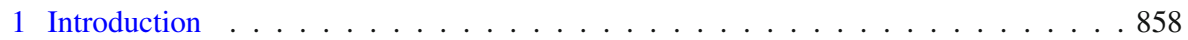

1.1 Related results . . . . . . . . . . . . . . . . . . . . . . . . . . 859

1.2 Diophantine inequalities . . . . . . . . . . . . . . . . . . . . . . . . . . . . . . .

1.3 Discussion of effective bounds and outline of the proofs . . . . . . . . . . . . 866

1.4 Organization of this paper . . . . . . . . . . . . . . . . . . 872

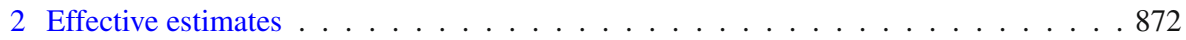

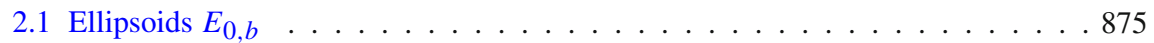

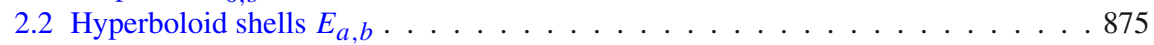

2.3 Quadratic forms of diophantine type $(\kappa, A) \ldots \ldots$. . . . . . . . . . . . . . . . . . . . .

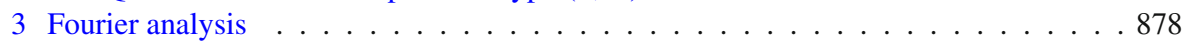

3.1 Smoothing . . . . . . . . . . . . . . . . . . . . . . . . . . . . . . . . . .

3.2 Fourier transforms and theta-series . . . . . . . . . . . . . . . 880

4 Functions on the space of lattices and geometry of numbers . . . . . . . . . . . . 889

4.1 Sympletic structure of $\Lambda_{t} \ldots \ldots \ldots \ldots$. . . . . . . . . . . . . . . . . . . . . . . . . .

4.2 Approximation by compact subgroups . . . . . . . . . . . . . . . . . . . . . . . . . . . . . . . . . . . . .

4.3 Irrational and diophantine lattices . . . . . . . . . . . . . . . . . . . . . . . . . . . . . . . . . . . . . . . .

5 Averages along translates of orbits of $\mathrm{SO}(2) \ldots \ldots . \ldots 904$

5.1 Application of geometry of numbers . . . . . . . . . . . . . . . . 904

5.2 Operators $A_{g}$ and functions $\tau_{\lambda}$ on $\operatorname{SL}(2, \mathbb{R}) \ldots \ldots$. . . . . . . . . . . . . . . . . . . . . .

5.3 Quasinorms and representations of $\operatorname{SL}(2, \mathbb{R}) \ldots \ldots . \ldots 916$

5.4 Functions $\alpha_{i}$ on the space of lattices and estimates for $A_{h} \alpha_{i} \ldots \ldots$. . . . 920

6 Proofs of Theorems 2.2 and $1.9 \ldots \ldots$. . . . . . . . . . . . . . . . . . . . . 924

7 Lattice point deficiency for admissible regions and applications . . . . . . . . . . . . 928

7.1 Smoothing of special parallelepiped regions . . . . . . . . . . . . . . . . . . 929

7.2 Fourier transform of weights for polyhedra . . . . . . . . . . . . . . . 934

7.3 Lattice point remainders for admissible parallelepipeds . . . . . . . . . . . . . 935

7.4 Applications of Theorem $2.2 \ldots \ldots$. . . . . . . . . . . . . . 943

8 Small values of quadratic forms at integer points . . . . . . . . . . . . . 950

8.1 Integer-valued quadratic forms . . . . . . . . . . . . . . . . . . . . . . . . . . . . . . . . . . .

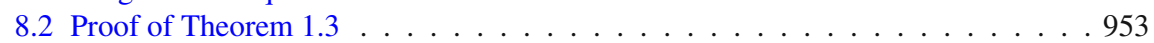

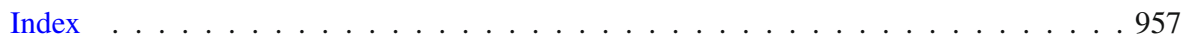

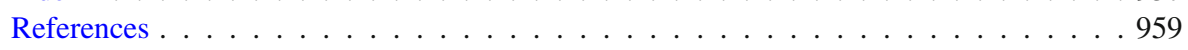

\section{Introduction}

Let $Q[x]$ denote an indefinite quadratic form in $d$ variables. We say that the form $Q$ is rational, if it is proportional to a form with integer coefficients; otherwise it is called irrational. The Oppenheim conjecture, proved by G. Margulis 
[40] in 1986, states that $Q\left[\mathbb{Z}^{d}\right]$ is dense in $\mathbb{R}$ if $d \geq 3$ and $Q$ is irrational. Initially this was conjectured for $d \geq 5$ by A. Oppenheim [47,48] in 1929 and in 1946 strengthened (for diagonal forms) to $d \geq 3$ by H. Davenport [18]. The proof given in 1986 uses a connection, noticed by M. S. Raghunathan, between the Oppenheim conjecture and questions concerning closures in $\operatorname{SL}(3, \mathbb{R}) / \operatorname{SL}(3, \mathbb{Z})$ of orbits of certain subgroups of $\operatorname{SL}(3, \mathbb{R})$. It is based on the study of minimal invariant sets and the limits of orbits of sequences of points tending to a minimal invariant set. Previous studies have mostly used analytic number theory methods. In fact, B. J. Birch, H. Davenport and D. Ridout proved in a series of papers that $Q\left[\mathbb{Z}^{d}\right]$ is dense in $\mathbb{R}$ if $d \geq 21$ provided that $Q$ is irrational, see $[39,41]$ for a complete historical overview until 1997.

For a measurable set $B \subset \mathbb{R}^{d}$ let vol $B$ denote the Lebesgue measure of $B$ and let $\operatorname{vol}_{\mathbb{Z}} B:=\#\left(B \cap \mathbb{Z}^{d}\right)$ denote the number of integer points in $B$. We define for $a, b \in \mathbb{R}$ with $a<b$ the hyperbolic shell

$$
E_{a, b} \stackrel{\text { def }}{=}\left\{x \in \mathbb{R}^{d}: a<Q[x]<b\right\} .
$$

The Oppenheim conjecture is equivalent to the statement that if $d \geq 3$ and $Q$ is irrational, then $\operatorname{vol}_{\mathbb{Z}} E_{a, b}=\infty$ whenever $a<b$. We would like to study the distribution of values of $Q$ at integer points, often referred to as "quantitative Oppenheim conjecture" with an emphasis on establishing effective error bounds for the approximation of the number of lattice points restricted to growing domains. Our methods rely mainly on Götze's Fourier approach [28] via Theta series, translating the lattice point counting problem into averages of certain functions on the space of lattices, for which we extend the mean-value estimates obtained by Eskin-Margulis-Mozes [23].

\subsection{Related results}

Let $\mathcal{R}$ be a continuous positive function on the sphere $\left\{v \in \mathbb{R}^{d}:\|v\|=1\right\}$ and let $\Omega=\left\{v \in \mathbb{R}^{d}:\|v\| \leq 1 / \mathcal{R}(v /\|v\|)\right\}$. Note that the Minkowski functional of $\Omega$, that is $M(v)=\inf \{r>0: v \in r \Omega\}$, may be rewritten as $M(v)=$ $\|v\| \mathcal{R}(v /\|v\|)$ and therefore $\Omega=\left\{v \in \mathbb{R}^{d}: M(v) \leq 1\right\}$. Without loss of generality we may assume that $\Omega \subset[-1,1]^{d}$. We denote by $r \Omega$ the dilate of $\Omega$ by $r>1$. In [20] S. G. Dani and G. Margulis obtained the following asymptotic exact lower bound under the same assumptions that $Q$ is irrational and $d \geq 3$ :

$$
\liminf _{r \rightarrow \infty} \frac{\operatorname{vol}_{\mathbb{Z}}\left(E_{a, b} \cap r \Omega\right)}{\operatorname{vol}\left(E_{a, b} \cap r \Omega\right)} \geq 1 .
$$


Remark 1.1 It is not difficult to prove (see Lemma 3.8 in [23]) that as $r \rightarrow \infty$,

$$
\operatorname{vol}\left(E_{a, b} \cap r \Omega\right) \sim \lambda_{Q, \Omega}(b-a) r^{d-2},
$$

where

$$
\lambda_{Q, \Omega} \stackrel{\text { def }}{=} \int_{L \cap \Omega} \frac{\mathrm{d} A}{\|\nabla Q\|}
$$

$L$ is the light cone $Q=0$ and $\mathrm{d} A$ is the area element on $L$.

The situation with asymptotics and upper bounds is more subtle. It was proved in [23] that if $Q$ is an irrational indefinite quadratic form of signature $(p, q)$ with $p+q=d, p \geq 3$ and $q \geq 1$, then for any $a<b$

$$
\lim _{r \rightarrow \infty} \frac{\operatorname{vol}_{\mathbb{Z}}\left(E_{a, b} \cap r \Omega\right)}{\operatorname{vol}\left(E_{a, b} \cap r \Omega\right)}=1
$$

or, equivalently, as $r \rightarrow \infty$

$$
\operatorname{vol}_{\mathbb{Z}}\left(E_{a, b} \cap r \Omega\right) \sim \lambda_{Q, \Omega}(b-a) r^{d-2},
$$

where $\lambda_{Q, \Omega}$ is as in (1.2).

If the signature of $Q$ is $(2,1)$ or $(2,2)$, then no universal formula like (1.4) holds. In fact, one can show (see Theorem 2.2 in [23]) that if $\Omega$ is the unit ball and $q=1$ or $q=2$, then for every $\varepsilon>0$ and every $a<b$ there exists an irrational quadratic form $Q$ of signature $(2, q)$ and a constant $c>0$ such that for an infinite sequence $r_{j} \rightarrow \infty$

$$
\operatorname{vol}_{\mathbb{Z}}\left(E_{a, b} \cap r_{j} \Omega\right)>c r_{j}^{d-2}\left(\log r_{j}\right)^{1-\varepsilon} .
$$

While the asymptotics as in (1.4) do not hold in the case of signatures $(2,1)$ and $(2,2)$, one can show (see [23]) that in these cases there is an upper bound of the form $r^{d-2} \log r$. This upper bound is effective and it is uniform over compact sets in the space of quadratic forms. In addition, there is an effective uniform upper bound (see [23]) of the form $c r^{d-2}$ for the case $p \geq 3, q \geq 1$.

The examples in [23] for the cases of signatures $(2,1)$ and $(2,2)$ are obtained by considering irrational forms which are very well approximated by split rational forms. More precisely, a quadratic form $Q$ is called extremely well approximable by split rational forms (EWAS) if for any $N>0$ there exists a split integral form $Q^{\prime}$ and a real number $t \geq 2$ such that

$$
\left\|t Q-Q^{\prime}\right\| \leq t^{-N},
$$


where $\|\cdot\|$ denotes a norm on the linear space of quadratic forms. It is shown in [22] that if $Q$ is an indefinite quadratic form of signature $(2,2)$, which is not (EWAS), then for any interval $(a, b)$, as $r \rightarrow \infty$,

$$
\widetilde{N}_{Q, \Omega}(a, b, r) \sim \lambda_{Q, \Omega}(b-a) r^{2},
$$

where $\lambda_{Q, \Omega}$ is the same as in (1.2) and $\tilde{N}_{Q, \Omega}(a, b, r)$ counts all the integral points in $E_{a, b} \cap r \Omega$ not contained in rational subspaces isotropic with respect to $Q$. It should be noted that

(i) an irrational quadratic form of signature $(2,2)$ may have at most four rational isotropic subspaces,

(ii) if $0 \notin(a, b)$, then $\widetilde{N}_{Q, \Omega}(a, b, r)=\operatorname{vol}_{\mathbb{Z}}\left(E_{a, b} \cap r \Omega\right)$.

The above mentioned results have analogs for inhomogeneous quadratic forms

$$
Q_{\xi}[x]=Q[x+\xi], \quad \xi \in \mathbb{R}^{d} .
$$

We define for $a, b \in \mathbb{R}$ with $a<b$ the shifted hyperbolic shell

$$
E_{a, b, \xi} \stackrel{\text { def }}{=}\left\{x \in \mathbb{R}^{d}: a<Q_{\xi}[x]<b\right\} .
$$

We say that $Q_{\xi}$ is rational if there exists $t>0$ such that the coefficients of $t Q$ and the coordinates of $t \xi$ are integers; otherwise $Q_{\xi}$ is irrational. Then, under the assumptions that $Q_{\xi}$ is irrational and $d \geq 3$, we have that (see [45])

$$
\liminf _{r \rightarrow \infty} \frac{\operatorname{vol}_{\mathbb{Z}}\left(E_{a, b, \xi} \cap r \Omega\right)}{\operatorname{vol}\left(E_{a, b, \xi} \cap r \omega\right)} \geq 1 .
$$

The proof of (1.6) is similar to the proof of (1.1).

Let $(p, q)$ be the signature of $Q$. If $p \geq 3, q \geq 1$ and $Q_{\xi}$ is irrational then

$$
\lim _{r \rightarrow \infty} \frac{\operatorname{vol}_{\mathbb{Z}}\left(E_{a, b, \xi} \cap r \Omega\right)}{\operatorname{vol}\left(E_{a, b, \xi} \cap r \Omega\right)}=1
$$

or, equivalently, as $r \rightarrow \infty$,

$$
\operatorname{vol}_{\mathbb{Z}}\left(E_{a, b, \xi} \cap r \Omega\right) \sim \lambda_{Q, \Omega}(b-a) r^{d-2} .
$$

The proof of (1.7) is similar to the proof of (1.3), see [45]. The latter paper [45] also contains an analog of (1.5) for inhomogeneous forms in the case of signature $(2,2)$. One should also mention related results of Marklof $[42,43]$. 
Remark 1.2 The proofs of the above mentioned results use such notions as a minimal invariant set (in the case of the Oppenheim conjecture) and an ergodic invariant measure. These notions do not have in general effective analogs. Because of that it is very difficult to get 'good' estimates for the size of the smallest non-trivial integral solution of the inequality $|Q[m]|<\varepsilon$ and 'good' error terms in the quantitative Oppenheim conjecture by applying dynamical and ergodic methods.

\subsection{Diophantine inequalities}

One of our main objective is to develop effective analogs of (1.8) and show that all indefinite quadratic forms $Q$ of rank at least 5 admit a non-trivial integral solution to the Diophantine inequality $|Q[m]|<\varepsilon$ whose size can be bounded effectively in terms of $\varepsilon^{-1}$. On the one hand, we will exploit Schlickewei's results [51] on small zeros of integral forms (see Sect. 8.1) in order to establish effective bounds depending on the signature $(r, s)$ of $Q$. On the other hand we will introduce an appropriate Diophantine condition on the space of quadratic forms, which will enable us to significantly improve our effective bounds due to the exponents appearing in the Diophantine approximation of $Q$. To state these bounds we need to introduce notation.

Denote by $Q$ also the symmetric matrix in $\operatorname{GL}(d, \mathbb{R})$ associated with the form $Q[x]:=\langle x, Q x\rangle$, where $\langle\cdot, \cdot\rangle$ is the standard Euclidean scalar product on $\mathbb{R}^{d}$. Let $Q_{+}$denote the unique positive symmetric matrix such that $Q_{+}^{2}=Q^{2}$ and let $Q_{+}[x]=\left\langle x, Q_{+} x\right\rangle$ denote the associated positive form with eigenvalues being the eigenvalues of $Q$ in absolute value. Let $q$, resp. $q_{0}$, denote the largest, resp. smallest, of the absolute value of the eigenvalues of $Q$ and assume $q_{0} \geq 1$. In the first case, where we compare $Q$ with rational forms, we can replace the form $Q$ by $Q / \varepsilon$ and consider the solubility of the inequality $|Q[m]|<1$. Since this Diophantine inequality includes the case of integral-valued indefinite forms, we shall appeal to Corollary 8.4 (a variant of Folgerung 3 in [51]) on the size of non-trivial integral solutions. Combining this result with our effective bounds we arrive at the following size estimate for a non-trivial solution of this Diophantine inequality. 
Theorem 1.3 For all indefinite and non-degenerate quadratic forms $Q$ of dimension $d \geq 5$ and signature $(r, s)$ there exists for any $\delta>0$ a non-trivial integral solution $m \in \mathbb{Z}^{d} \backslash\{0\}$ to the Diophantine inequality $|Q[m]|<1$ satisfying

$$
\left\|Q_{+}^{1 / 2} m\right\| \ll_{\delta, d}\left(q / q_{0}\right)^{\frac{d+1}{d-2}} q^{\frac{1}{2}+\max \{\rho d+2, d+1\} /(d-4)+\delta},
$$

where the dependency on the signature $(r, s)$ is given by

$$
\rho:=\rho(r, s):= \begin{cases}\frac{1}{2} \frac{r}{s} & \text { for } r \geq s+3 \\ \frac{1}{2} \frac{s+2}{s-1} & \text { for } r=s+2 \text { or } r=s+1 \\ \frac{1}{2} \frac{s+1}{s-2} & \text { for } r=s .\end{cases}
$$

In particular, for indefinite non-degenerate forms in $d \geq 5$ variables of signature $(r, s)$ and eigenvalues in absolute value contained in a compact set $[1, C]$, i.e $1 \leq q_{0} \leq q \leq C$, Theorem 1.3 yields non-trivial solutions $m \in \mathbb{Z}^{d}$ of $|Q[m]|<\varepsilon$ of size bounded by

$$
\|m\| \ll_{C, \delta} \varepsilon^{-\max \{\rho d+2, d+1\} /(d-4)-\delta} .
$$

As an example, we obtain solutions of order $\ll_{C, \delta} \varepsilon^{-1-\frac{5}{(d-4)}-\delta}$ for the special case $r=s+3$ and $d \geq 12$. More generally, we may embed $\mathbb{Z}^{d_{1}} \subset \mathbb{Z}^{d}$ for dimensions $d \geq d_{1} \geq 5$, in such a way that the restricted form is indefinite and of rank $d_{1}$, and apply Theorem 1.3 to this form in $d_{1}$ dimensions. As a consequence, since $\left(Q^{*}\right)^{2} \leq Q^{2}$ in the ordering of positive forms we get $q \geq q^{*} \geq q_{0}^{*} \geq q_{0} \geq 1$ and $\left|\operatorname{det} Q^{*}\right| \leq|\operatorname{det} Q|$, we obtain the following corollary.

Corollary 1.4 For all indefinite and non-degenerate quadratic forms $Q$ in $d \geq 5$ variables there exists for any $\varepsilon>0$ at least one non-trivial integral solution $m \in \mathbb{Z}^{d}$ of

$$
\begin{aligned}
|Q[m]| & <\varepsilon, \\
\|m\| & \leq c_{C, \delta} \varepsilon^{-f_{d}-\delta},
\end{aligned}
$$

for any $\delta>0$, where $f_{d}=12,8 \frac{1}{2}, 7 \frac{2}{3}$ for $d=5,6,7$ respectively and $f_{d}=7 \frac{1}{2}$ for all $d \geq 8$. The constant $c_{C, \delta}$ depends only on $\delta$ and $C>0$ for forms $Q$ satisfying $1 \leq q_{0} \leq q \leq C$.

Remark 1.5 (a) For the special case of diagonal indefinite forms $Q[x]=$ $\sum_{j=1}^{5} q_{j} x_{j}^{2}$ with $\min \left|q_{j}\right| \geq 1$ Birch and Davenport [3], obtained a sharper bound. They showed for arbitrary small $\delta>0$ that there exists an $m \in \mathbb{Z}^{5} \backslash\{0\}$ 
with $|Q[m]|<1$ and $Q_{+}[m] \ll_{d, \delta}|\operatorname{det} Q|^{1+\delta}$. This implies (as above) for a compact set of forms $Q$ that there exists an integral vector $m$ satisfying $|Q[m]|<\varepsilon$ and $\|m\| \leq c_{d, \delta} \varepsilon^{-2+\delta}$ for any fixed $\delta>0$. In [7] Buterus, Götze and Hille extended the approach of Birch and Davenport to improve the size of a solution by using Schlickewei's result [51] on small zeros of integral forms: Let $Q[x]=\sum_{j=1}^{d} q_{j} x_{j}^{2}$ be an indefinite form of signature $(r, s)$ in $d=r+s \geq 5$ variables. Then for any $\varepsilon>0$ the Diophantine inequality $|Q[m]|<\varepsilon$ admits a non-trivial solution $m \in \mathbb{Z}^{d}$, whose size is bounded by $\ll \varepsilon^{-\rho+\delta}$ for any fixed $\delta>0$.

(b) Recently, quantitative versions of the Oppenheim conjecture were studied by Bourgain [9], Athreya and Margulis [1], and Ghosh and Kelmer [26]. Bourgain [9] proves essentially optimal results for one-parameter families of diagonal ternary indefinite quadratic forms under the Lindelöf hypothesis by using also a Fourier approach, based on Epstein-Zeta functions. In contrast, Ghosh and Kelmer [26] consider the space of all indefinite ternary quadratic forms and use spectral methods (an effective mean ergodic theorem). Lastly, Athreya and Margulis apply classical bounds of Rogers for $L^{2}$-norm of Siegel transforms in order to prove that for every $\delta>0$ and almost every $Q$ (with respect to the Lebesgue measure) with signature $(r, s)$, there exists a non-trivial integral solution $m \in \mathbb{Z}^{d}$ to the Diophantine inequality $|Q[m]|<\varepsilon$ whose size is bounded by $\|m\| \ll \delta, Q \varepsilon^{-\frac{1}{d-2}-\delta}$ if $d \geq 3$.

As mentioned above let us introduce a class of Diophantine forms as follows.

Definition 1.6 We call $Q$ Diophantine of type $(\kappa, A)$, where $\kappa, A>0$, if for any $m \in \mathbb{Z} \backslash\{0\}$ and $M \in M(d, \mathbb{Z})$ we have

$$
\inf _{t \in[1,2]}\|M-m t Q\| \geq A|m|^{-\kappa},
$$

where $\|\cdot\|$ denotes the operator norm induced by the Euclidean norm on $\mathbb{R}^{n}$.

We shall see in Sect. 4.3 that almost every form satisfies this property for some $\kappa$ and $A$. In particular, fixing an integer $k$ such that $1 \leq k \leq \frac{d(d+1)}{2}-1$, we shall show that a form $Q$ for which $k+1$ non-zero entries $y, x_{1}, \ldots, x_{k}$ exist such that $x_{1} / y, \ldots, x_{k} / y$ are algebraic and $1, x_{1} / y, \ldots, x_{k} / y$ are linearly independent over $\mathbb{Q}$ is Diophantine in this sense and admits a non-trivial solution to the Diophantine inequality $|Q[m]|<\epsilon$ of order $\ll_{Q, d, \delta} \epsilon^{-\frac{d(3+2 k)-4}{2 k(d-4)}-\delta}$ for any $\delta>0$. In particular, for $k=\frac{d(d+1)}{2}-1$ we can give a bound for the size of the least solution of order $\ll_{Q, d, \delta} \epsilon^{-\frac{d^{3}+d^{2}+d-4}{\left(d^{2}+d-2\right)(d-4)}-\delta}$ and in this case for $d=5$ of order $\ll_{Q, \delta} \epsilon^{-151 / 28-\delta}$. 
Corollary 1.7 Let $Q$ be an indefinite quadratic form in $d \geq 5$ variables and of Diophantine type $(\kappa, A)$ and fix $\delta>0$. Then for any $\varepsilon>0$ there exists $a$ non-trivial lattice point $m \in \mathbb{Z}^{d} \backslash 0$ satisfying

$$
|Q[m]|<\varepsilon \text { and }\|m\| \ll_{Q, d, \delta} \varepsilon^{-\frac{2 d+3 \kappa d-4 \kappa}{2 d-8}-\delta} .
$$

For irrational indefinite quadratic forms we may quantify the density of values $Q[m], m \in r \Omega \cap \mathbb{Z}^{d}$, where $\Omega$ denotes a (not necessarily admissible) parallelepiped satisfying (7.1) (see Sect. 7.3) as follows: Consider the set

$$
V(r) \stackrel{\text { def }}{=}\left\{Q[m]: m \in r \Omega \cap \mathbb{Z}^{d}\right\} \cap\left[-c_{0} r^{2}, c_{0} r^{2}\right]
$$

of values of $Q[x], x \in r \Omega \cap \mathbb{Z}^{d}$ lying in the interval $\left[-c_{0} r^{2}, c_{0} r^{2}\right]$, where $c_{0}$ denotes the constant introduced in Lemma 7.1. For each $r \geq 1$ we arrange the values $V(r)$ in increasing order $v_{0}(r)<\ldots<v_{k}(r), k=k(r)$, and define the maximal gap between successive values of $V(r)$ as

$$
d(r):=\sup _{i \in\{1, \ldots, k(r)\}}\left|v_{i}(r)-v_{i-1}(r)\right| .
$$

As a consequence of our technical quantitative bounds we obtain

Corollary 1.8 Let $Q$ denote a non-degenerate indefinite form in $d \geq 5$ variables and of Diophantine type $(\kappa, A)$. For $\delta>0$ we obtain for the maximal gap $d(r)$ between successive values of the quadratic form in the set $V(r)$

$$
d(r) \leq r^{-\nu_{0}+\delta},
$$

for sufficiently large $r \geq c_{\delta, d, \Omega, \kappa, A, Q}$, where $v_{0}:=\frac{2 d-8}{2 d+3 \kappa d-4 \kappa}$ and $c_{\delta, d, \Omega, \kappa, A, Q}>0$ denotes a constant depending on $\kappa, A, Q, \Omega, d$ and $0<$ $\delta<1 / 10$ (here we omit a description of the explicit dependence).

For positive definite quadratic forms Davenport and Lewis (see [19]) conjectured, that the distance between successive values $v_{n}$ of the quadratic form $Q[x]$ on $\mathbb{Z}^{d}$ converges to zero as $n \rightarrow \infty$, provided that the dimension $d$ is at least five and $Q$ is irrational. This conjecture was proved by Götze in [28]. It also follows by the results of the present paper which provides error bounds for the lattice point counting problem for the indefinite case as well as the positive definite case.

The proof is similar as in the case of positive forms solved in [28]: For any $\varepsilon>0$ and any interval $[b, b+\varepsilon]$, we find at least two lattice points in the shell $E_{b, b+\varepsilon}$ (and the box of size $r=\sqrt{2 b}$ ) by Corollary 2.4, provided that $b$ is larger than a threshold $b(\varepsilon)$. Here $b(\varepsilon)$ and consequently the distance between successive values (as a function of $b$ ) depends on the rate of convergence of 
the Diophantine characteristic $\rho_{Q}^{\text {ell }}(r)$ in the bound of Corollary 2.4 towards zero. For quadratic forms of Diophantine type $(\kappa, A)$ this dependency can be stated explicitly.

\subsection{Discussion of effective bounds and outline of the proofs}

In order to prove an effective result like Theorem 1.3 we need an explicit bound for the error, say $R\left(I_{E_{a, b} \cap r \Omega}\right)$ (for a formal definition see (1.15) below) with $I_{B}$ denoting the indicator of a set $B$, of approximating the number of integral points $m \in E_{a, b}$ in a bounded domain $r \Omega$ by the volume vol $\left(E_{a, b} \cap r \Omega\right)$, compare Remark 1.1. First, we simplify the problem by replacing the weights $I_{r \Omega}(m)=1$ of integral points $m \in r \Omega$ by suitable smoothly changing weights $\mathrm{v}(m / r)$ (for notational simplicity, we will write $\mathrm{v}_{r}(m):=\mathrm{v}(m / r)$ ), which tend to zero as $m / r$ tends to infinity. This smoothing (together with a smoothing of the indicator function of $[a, b]$ ) allows us to use techniques from Fourier analysis, but we are forced to restrict the region $\Omega$ to parallelepipeds in order to ensure that the corresponding error has logarithmic growth only.

\subsubsection{Fourier analysis}

Starting with smooth weight functions $\mathrm{v}_{r}$ (which depend on the dilation parameter $r$ ), we also construct a $w$-smoothing $g$ of the indicator function of $[a, b]$ via convolution with an appropriate kernel $k$ whose Fourier transform decays like $|\widehat{k}(t)| \ll \exp \{-\sqrt{|w t|}\}$. This allows us to replace the indicator function of $[a, b]$ in the lattice point counting problem by a smooth function, gaining an error bounded in Corollary 3.2. After this smoothing procedure, writing $g^{Q}(x):=g(Q[x])$, our main objective will be to estimate the weighted lattice remainder

$$
R\left(g^{Q} \mathrm{v}_{r}\right) \stackrel{\text { def }}{=} \sum_{m \in \mathbb{Z}^{d}} g(Q[m]) \mathrm{v}\left(\frac{m}{r}\right)-\int_{\mathbb{R}^{d}} g(Q[x]) \mathrm{v}\left(\frac{m}{r}\right) \mathrm{d} x,
$$

where $g$ and $\mathrm{v}$ are smooth functions whose Fourier transforms decay fast enough as well. More precisely, we will assume that v satisfies (2.4). (At this point we should note that the abbreviation introduced in (1.15) will frequently be used to denote remainder terms.) Next we shall use inverse Fourier transforms in order to express the weights as

$$
g(Q[m])=\int_{\mathbb{R}} \widehat{g}(t) \exp \{2 \pi \mathrm{i} t Q[m]\} \mathrm{d} t, \quad \zeta(m)=\int_{\mathbb{R}^{d}} \widehat{\zeta}(u) \exp \{2 \pi \mathrm{i}\langle u, m\rangle\} \mathrm{d} u,
$$

where $\zeta(x)=\mathrm{v}(x) \exp \left\{Q_{+}[x]\right\}$. Combining the resulting factors $\exp \{2 \pi \mathrm{i} t$ $Q[m]\}, \exp \{2 \pi \mathrm{i}\langle v, m\rangle\}$ and $\exp \left\{-Q_{+}\left[\frac{x}{r}\right]\right\}$ in (1.15) into terms of the generalized theta series 


$$
\theta_{v}(t) \stackrel{\text { def }}{=} \sum_{m \in \mathbb{Z}^{d}} \exp \left\{-2 \pi \mathrm{i}\langle v, m\rangle / r-2 \pi \mathrm{i} t Q[m]-Q_{+}[m] / r^{2}\right\}
$$

one arrives at an expression for the sum $V_{r}:=\sum_{m \in \mathbb{Z}^{d}} \mathrm{v}\left(\frac{m}{r}\right) g(Q[m])$ by the following integral (in $t$ and $v$ ) over $\theta_{v}(t)$ :

$$
V_{r}=\int_{\mathbb{R}^{d}} \widehat{\zeta}(v) \int_{\mathbb{R}} \widehat{g}(t) \theta_{v}(t) \mathrm{d} t \mathrm{~d} v .
$$

The approximating integral $W_{r}:=\int_{\mathbb{R}^{d}} \mathrm{~V}\left(\frac{m}{r}\right) g(Q[x]) \mathrm{d} x$ to this sum $V_{r}$ can be rewritten in exactly the same way by means of the theta integral

$$
\vartheta_{v}(t) \stackrel{\text { def }}{=} \int_{\mathbb{R}^{d}} \exp \left\{-2 \pi \mathrm{i}\langle v, x\rangle / r-2 \pi \mathrm{i} t Q[x]-Q_{+}[x] / r^{2}\right\} \mathrm{d} x,
$$

replacing the theta $\operatorname{sum} \theta_{v}(t)$. Thus, in order to estimate the error $\left|R\left(g^{Q} \mathrm{v}_{r}\right)\right|=$ $\left|V_{r}-W_{r}\right|$, the integral over $t$ and $v$ of $\left|\theta_{v}(t)-\vartheta_{v}(t)\right||\widehat{g}(t) \widehat{\zeta}(v)|$ has to be estimated.

For $|t| \leq q_{0}^{-1 / 2} r^{-1}$ and $\|x\| \ll r$ the functions $x \mapsto \exp \{2 \pi \mathrm{i} t Q[x]\}$ are sufficiently smooth, so that the sum $\theta_{v}(t)$ is well approximable by the first term of its Fourier series, that is the corresponding integral $\vartheta_{v}(t)$, see (3.16) and (3.33). The error of this approximation, after integration over $v$, yields the second error term in (1.26), which does not depend on the Diophantine properties of $Q$. Additionally, we may restrict the integration to $|t| \leq T_{+}$for an appropriate choice of $T_{+}$(depending on the width of the shell) by using the decay rate of the kernel $k$. So we end up with the remaining error term

$$
I=\int_{T_{+}>|t|>q_{0}^{-1 / 2} r^{-1}} \int_{\mathbb{R}^{d}}\left|\theta_{v}(t) \widehat{g}(t) \widehat{\zeta}(v)\right| \mathrm{d} v \mathrm{~d} t,
$$

which we estimate as follows

$$
I \leq\|\widehat{\zeta}\|_{1} \sup _{v \in \mathbb{R}^{d}} \int_{T_{+} \geq|t|>q_{0}^{-1 / 2} r^{-1}}\left|\theta_{v}(t)\right||\widehat{g}(t)| \mathrm{d} t
$$

The second factor in the bound of $I$ in (1.18) encodes both the Diophantine behavior of $Q$ as described above as well as the growth rate with respect to $r$. We shall describe in the next subsection our method to extract out of this factor the correct rate of growth, while simultaneously avoiding the loss of information on the Diophantine properties of $Q$, provided that $d>4$. However, let us first state that the resulting bound (the choice of $T_{+}$depending on the width of the shell) is an error bound depending on characteristics of $\widehat{\zeta}(v)$ of the form (see Theorem 2.2) 


$$
R\left(I_{E_{a, b}} \mathrm{v}_{r}\right) \ll_{\kappa, d, Q}, w r^{d-2}+\|\widehat{\zeta}\|_{1} \rho_{Q, b-a}^{w}(r) r^{d-2}+\|\widehat{\zeta}\|_{1, *} r^{d / 2} \log \left(1+\frac{b-a}{r}\right),
$$

which has to be optimized in the smoothing size $w$ (compare e.g. Corollary 2.4) and $\rho_{Q, b-a}^{w}(r)$ depends on the Diophantine properties of $Q$ and $r$ (see Theorem 2.2).

\subsubsection{Mean-value estimates}

In order to describe the second term in (1.19), we follow [28] (by using a modified Weyl differencing argument) to show in Lemma 3.3 that uniformly in $v$ and pointwise in $t$

$$
\left|\theta_{v}(t)\right|^{2} \ll r^{d}|\operatorname{det} Q|^{-1 / 2} \sum_{v \in \Lambda_{t}} \exp \left\{-\|v\|^{2}\right\},
$$

where $\left\{\Lambda_{t}\right\}_{t \in \mathbb{R}}$ is a family of $2 d$-dimensional unimodular lattices generated by orbits of one-parameter subgroups of $\operatorname{SL}(2, \mathbb{R})$ indexed by $t$ and $r$, see (3.47) for the precise definition. It is well-known that the expression $\psi(r, t):=$ $\sum_{v \in \Lambda_{t}} \exp \left\{-\|v\|^{2}\right\}$ can be bounded by the number of lattice points $v \in \Lambda_{t}$ satisfying $\|v\|_{\infty} \ll 1$. Combining this estimate together with the symplectic structure of $\Lambda_{t}$ (see Sect. 4.1) yields the estimate

$$
\psi(r, t) \ll \frac{1}{M_{1}\left(\Lambda_{t}\right) \ldots M_{d}\left(\Lambda_{t}\right)} \asymp_{d} \alpha_{d}\left(\Lambda_{t}\right),
$$

where $M_{i}\left(\Lambda_{t}\right)$ denotes the $i$-th successive minima of $\Lambda_{t}$ and $\alpha_{d}\left(\Lambda_{t}\right)$ the $d$-th $\alpha$-characteristic of $\Lambda_{t}$, that is $\alpha_{d}\left(\Lambda_{t}\right)=\sup \left\{\left|\operatorname{det}\left(\Lambda^{\prime}\right)\right|^{-1}: \Lambda^{\prime}\right.$ is a $\mathrm{d}$-dimensional sublattice of $\left.\Lambda_{t}\right\}$. After a local approximation of a certain oneparameter unipotent subgroup by the compact group $\mathrm{SO}(2)$ (see Sect. 4.2), we estimate the average of $\alpha_{d}\left(\Lambda_{t}\right)^{\beta}$ over $t$ for $0<\beta \leq 1 / 2$ in Lemmas 5.12, 6.1 and 6.2. This argument involves a recursion in the size of $r$ and builds upon a method developed in [23] on upper estimates of averages of certain functions on the space of lattices along translates of orbits of compact subgroups.

Let us give a brief sketch of the main ideas involved in this argument. Let $\mathrm{G}=\mathrm{SL}(2, \mathbb{R}), \mathrm{K}=\mathrm{SO}(2)$ endowed with the probability Haar measure $\mathrm{d} k$ and denote by $A_{r}$ the mean-value operator on $\mathrm{K} \backslash \mathrm{G}$ defined by

$$
A_{r}(f)(h)=\int_{\mathrm{K}} f(g k h) \mathrm{d} k,
$$

where $f$ is any continuous function on $\mathrm{K} \backslash \mathrm{G}, g \in \mathrm{G}$ denotes any element for which $\|g\|=r$ and $\|\cdot\|$ denotes the operator norm induced by the standard Euclidean norm. Fixing $2 / d<\beta \leq 1 / 2$, we shall show that uniformly in $v$ 
and for all intervals $I$ of fixed bounded length there exists a positive function $f$ depending only on $Q$ and $\beta$ such that

$$
\int_{I}\left|\theta_{v}(t)\right| \mathrm{d} t \ll r^{d-\beta d}|\operatorname{det} Q|^{-1 / 4} \gamma_{I, \beta}(r) A_{r}(f)(\mathbb{1}),
$$

where $\gamma_{I, \beta}(r)$ contains information on the Diophantine properties of $Q$ and tends to zero for irrational forms as $r$ tends to infinity (see Corollary 4.11).

The function $f$ does not appear isolated but emerges as the maximum of a family of positive functions $f_{1}, \ldots, f_{2 d}$. For a positive number $r_{0}>0$ and any $g_{0} \in \mathrm{G}$ such that $\left\|g_{0}\right\|=r_{0}$ we show that this family satisfies two main properties. First, the value of each $f_{i}$ on any orbit of the form $g_{0} \mathrm{~K} h$ is bounded (up to a constant depending only on $r_{0}$ ) by its value at $f_{i}(h)$. Second, the mean-value $A_{r_{0}}\left(f_{i}\right)$ of any $f_{i}$ satisfies the following functional inequality (see Lemma 5.11)

$$
A_{r_{0}} f_{i} \ll \tau_{\lambda_{i}}\left(g_{0}\right) f_{i}+\max _{0<j \leq \bar{i}} \sqrt{f_{i-j} f_{i+j}}
$$

where we set $\bar{i}=\min \{i, 2 d-i\}, \lambda_{i}:=\max \{2, \beta \bar{i}\}$ and $\tau_{\lambda_{i}}$ denotes the spherical function

$$
\tau_{\lambda_{i}}(g)=\int_{\mathrm{K}}\left\|g k e_{1}\right\|^{-\lambda_{i}} \mathrm{~d} k
$$

where $e_{1}=(1,0)$ denotes the first standard unit vector on $\mathbb{R}^{2}$.

The asymptotic growth of spherical functions is well-understood and in our case $\tau_{\lambda}(g) \asymp\|g\|^{\lambda-2}$ whenever $\lambda>2$ and $g \notin \mathrm{K}$. Here spherical functions are crucial precisely because they are the eigenfunctions of the mean-value operator. We show, in a first instance, that any positive function $f$ satisfying an inequality of the form

$$
A_{r_{0}} f \ll \tau_{\lambda}\left(g_{0}\right) f+b \tau_{\eta},
$$

for $\lambda>2$ and $0<\eta<\lambda$ satisfies

$$
A_{r} f(\mathbb{1}) \ll \tau_{\lambda}(g) f(\mathbb{1}),
$$

for any $r>0$, where $g \in \mathrm{G}$ is any element for which $\|g\|=r$. In other words, the growth of the mean value at $\mathbb{1}$ grows at most as fast as the associated spherical function. In a second instance we obtain, after radializing the family, a preliminary estimate of the form 


$$
A_{r}(f)(\mathbb{1}) \ll_{\mu} f(\mathbb{1}) \tau_{\mu}(g),
$$

for any fixed $\mu>\lambda_{d}$. We then show inductively, using repeatedly (1.21), (1.22) and (1.23) that

$$
A_{r}\left(f_{i}\right) \ll f(\mathbb{1}) \tau_{\mu_{i}} \text {, for all } i \neq d
$$

for an appropriate sequence $\lambda_{d}>\mu_{i}>\lambda_{i}$. Combining these estimates again with (1.21) in the case $i=d$ then yields the inequality $A_{r_{0}} f_{d} \ll \tau_{\lambda_{d}}\left(g_{0}\right) f_{d}+$ $f(\mathbb{1}) \tau_{\eta}$, for some $\eta<\lambda_{d}$, which implies together with (1.21) and (1.24) the desired and expected estimate (see Theorem 5.12), namely that $A_{r}(f)(\mathbb{1}) \ll$ $\tau_{\lambda_{d}}(g) f(\mathbb{1}) \asymp r^{\beta d-2} f(\mathbb{1})$ for any $r \gg 1$ and any $g \in \mathrm{G}$ for which $\|g\|=r$. In particular for any such interval $I$ we obtain the following bound

$$
\int_{I}\left|\theta_{v}(t)\right| \mathrm{d} t \ll r^{d-2}|\operatorname{det} Q|^{-1 / 4} \gamma_{I, \beta}(r) f(\mathbb{1}) .
$$

At this point the current approach is fundamentally different to the approach of previous effective bounds for $R\left(I_{E_{a, b} \cap r \Omega}\right)$ by Bentkus and Götze [6] (see also [5]) valid for $d \geq 9$ and positive as well as indefinite forms. The reduction to (1.20) and the Diophantine factor $\rho_{Q, b-a}^{w}(r)$ follows the approach used by Götze in [28], where the average on the right-hand side of (1.20) was estimated for $d \geq 5$ by methods from the Geometry of Numbers and essentially required positive definite forms. A variant of that method was applied to split indefinite forms in a PhD thesis by G. Elsner [21].

\subsubsection{Smooth weights on $\mathbb{Z}^{d}$}

For the Gaussian weights $\mathrm{v}_{r}(x)=\exp \left\{-2 Q_{+}[x] / r^{2}\right\}$ our techniques yield effective bounds for the approximation of a weighted count of lattice points $m \in \mathbb{Z}^{d}$ with $Q[m] \in[a, b]$ by a corresponding integral with an error

$$
R\left(I_{E_{a, b}} \mathrm{v}_{r}\right)=\sum_{m \in E_{a, b} \cap \mathbb{Z}^{d}} \mathrm{v}_{r}(m)-\int_{E_{a, b}} \mathrm{v}_{r}(x) \mathrm{d} x .
$$

The following bounds for $R\left(I_{E_{a, b}} \mathrm{v}_{r}\right)$ are identical for the case of positive and indefinite $d$-dimensional forms $Q$, provided that $d \geq 5$. Using Vinogradov's notation $A \ll_{B} C$, meaning that $A<c_{B} C$ with a constant $c_{B}>0$ depending on $B$, we have 
Theorem 1.9 Let $Q$ be a non-degenerate quadratic form in $d \geq 5$ variables. Choose $\beta=\frac{2}{d}+\frac{\delta}{d}$ for some arbitrary small $\delta \in\left(0, \frac{1}{10}\right)$. Then for any $r \geq q^{1 / 2}$, where $q$ denotes the maximal eigenvalue of $Q, b>a$ and $0<w<(b-a) / 4$ we have

$$
R\left(I_{E_{a, b}} \mathrm{v}_{r}\right) \ll_{Q, \beta, d} r^{d-2}\left(w+\rho_{Q, b-a}^{w}(r)\right)+r^{d / 2-1}(b-a),
$$

provided that $b-a \leq r$. If $r<b-a \ll r^{2}$ the second term in the bound has to be replaced by $r^{d / 2} \log r$.

In Theorem 2.2 an explicit description of the Diophantine factor $\rho_{Q, b-a}^{w}(r)$ will be provided. Depending on whether $Q$ is definite or indefinite, this factor will be further refined in Corollary 2.4, resp. Corollary 2.5. Moreover, the function $\rho_{Q, b-a}^{w}(r)$ tends to zero as $r$ tends to infinity if $Q$ is irrational. Additionally, if $Q$ is Diophantine of type $(\kappa, A)$, as we shall introduce in Definition 1.6, we find a polynomial decay $\rho_{Q, b-a}^{w}(r) \ll_{Q, d, A} r^{-v}$ for an appropriate choice of $0<w<(b-a) / 4$, where $v \in(0, \infty)$ depends on $d, \kappa$ and $A$, see Corollary 2.6. These results follow from Theorem 2.2 with parameters chosen for the indefinite, positive and effective Diophantine cases in the proofs in Sect. 7.4.

\subsubsection{The role of the region $\Omega$}

In order to estimate the lattice point deficiency $R\left(I_{E_{a, b} \cap r \Omega}\right)$ we have to $\varepsilon$ smooth the indicator function of $\Omega$ which yields weights $\zeta=\zeta_{\varepsilon}$ and an additional error of order $\varepsilon(b-a) r^{d-2}$ in case of indefinite forms due to the intersection of $E_{a, b}$ with the boundary $\partial r \Omega$. For positive definite forms, $r \Omega$ contains $E_{a, b}$, that is $\varepsilon>0$ could be fixed independent of $r$, since this boundary intersection term is not present here.

In the indefinite case one needs to match the actual size of the error by choosing $\varepsilon$ small enough in (1.19). This leads to a critical dependence on $\varepsilon$ through the Fourier transform of $\zeta_{\varepsilon}$ and its characteristics (see (2.6)). Here $\left\|\widehat{\zeta_{\varepsilon}}\right\|_{1}$ moderately grows like $(\log 1 / \varepsilon)^{d}$ for arbitrary small $\varepsilon$ in the case of polyhedra only, see Lemma 7.2. The dependence of $\left\|\widehat{\zeta}_{\varepsilon}\right\|_{1, *}$, see (2.6), is again critically dependent on $\Omega$ and the width $b-a$ of the hyperbolic shell $E_{a, b}$. For $b-a \gg r$ the boundary of $r \Omega \cap E_{a, b}$ will contain a larger segment of $\partial r \Omega$. For a sequence of scalings $r$ these segments of the $(d-1)$-polytope potentially contain a large number of lattice points which induce large errors in the lattice point approximation, for which the technical restriction to the region $\Omega$ is solely responsible. In order to avoid this artefact which is reflected by a large growth of $\left\|\widehat{\zeta}_{\varepsilon}\right\|_{1, *}$ when $\varepsilon$ is small, we restrict ourselves to special admissible regions $r \Omega$, where $\Omega=B^{-1}[-1,1]^{d}$, and $B \in \operatorname{GL}(d, \mathbb{R})$ is chosen such that the lattice $\Gamma=B \mathbb{Z}^{d}$ is admissible in the sense of Sect. 7.3, i.e. both (7.1) and (7.29) are satisfied. This ensures that the lattice point remainder of $r \Omega$ satisfies 
$\left|\operatorname{vol}_{\mathbb{Z}} r \Omega-\operatorname{vol} r \Omega\right| \ll \Omega(\log r)^{d-1}$ uniformly which is 'abnormally' small. Likewise $\left\|\widehat{\zeta}_{\varepsilon}\right\|_{1, *}$ grows of order $(\log 1 / \varepsilon)^{d}$ only. The resulting error bounds in Corollary 2.5 for wide shells with $\max \{|a|,|b|\} \ll_{B} r^{2}$ are then comparable up to at most $(\log 1 / \varepsilon)^{d}$ factors to the case of positive forms in Corollary 2.4.

\subsection{Organization of this paper}

The paper is organized mostly in logical order. In Sect. 2 we describe the explicit technical estimates on lattice point remainders for both positive definite and indefinite forms. In the following Sect. 3 we transfer the problem to Fourier transforms of the error starting with a first smoothing step and rewrite the lattice remainder in terms of integrals over $d$-dimensional theta sums. Section 4 provides a reformulation of the problem via upper bounds in terms of integrals over the absolute value of other theta sums with an underlying symplectic structure on $\mathbb{R}^{2 d}$ which, in turn, are estimated using basic arguments from the Geometry of Numbers. Section 5 contains crucial estimates for averages of functions on the space of lattices. Finally, in Sect. 6 all these results are combined to prove Theorem 2.2. Starting with the applications, we collect in Sect. 7 the geometric bounds related to parallelepiped regions $\Omega$ used in this paper and afterwards conclude (in Sect. 7.4) the results of Sect. 2. In the last Sect. 8 we focus on small values of indefinite quadratic forms: After recollecting and refining some results due to Schlickewei [51] on the size of small zeros of integral quadratic forms, we shall prove Theorem 1.3.

Compared to an earlier preprint [27] this version has been rewritten so that it allows to separate the error contributions due to the Diophantine properties of $Q$ and the influence of weights for the lattice points in Theorem 2.2. The latter has been developed for special choices of regions $\Omega$ which are particularly relevant for wide shells $E_{a, b}$ in Sect. 7. Moreover, the effective bounds for nontrivial solutions of the Diophantine inequality $|Q[m]|<\varepsilon$ have been improved in terms of the signature $(r, s)$ by using Schlickewei's result [51] on small zeros of quadratic forms. In addition, we included a number of corrections concerning the explicit dependence on $Q$ (resp. $\Omega$ ) and the dimensions, and corrected typos as well.

\section{Effective estimates}

We consider the quadratic form

$$
Q[x] \stackrel{\text { def }}{=}\langle x, Q x\rangle \text { for } x \in \mathbb{R}^{d},
$$

where $\langle\cdot, \cdot\rangle$ resp. $\|\cdot\|$ denote the standard Euclidean scalar product and norm, $Q: \mathbb{R}^{d} \rightarrow \mathbb{R}^{d}$ denotes a symmetric linear operator in $\operatorname{GL}(d, \mathbb{R})$ with eigenvalues $q_{1}, \ldots, q_{d}$. Write 


$$
q_{0} \stackrel{\text { def }}{=} \min _{1 \leq j \leq d}\left|q_{j}\right|, \quad q \stackrel{\text { def }}{=} \max _{1 \leq j \leq d}\left|q_{j}\right|, \quad d_{Q} \stackrel{\text { def }}{=}|\operatorname{det} Q|^{-1 / 2} .
$$

In what follows we shall always assume that the form is non-degenerate, that is $q_{0}>0$. In order to describe the explicit bounds we need to introduce some more notations. Let $\beta>\frac{2}{d}$ such that $0<\frac{1}{2}-\beta<\frac{1}{2}-\frac{2}{d}$ for $d>4$. For a lattice $\Lambda \subset \mathbb{R}^{n}, n \in \mathbb{N}$, with $\operatorname{dim} \Lambda=n$ we define for $1 \leq l \leq n$ its $\alpha_{l}$-characteristic by

$$
\alpha_{l}(\Lambda) \stackrel{\text { def }}{=} \sup \left\{\left|\operatorname{det}\left(\Lambda^{\prime}\right)\right|^{-1}: \Lambda^{\prime} \subset \Lambda, \quad l \text {-dimensional sublattice of } \Lambda\right\} .
$$

Here $\Lambda^{\prime}=B \mathbb{Z}^{n}$ is determined by a $n \times l$-matrix $B$ and $\operatorname{det}\left(\Lambda^{\prime}\right)=\operatorname{det}\left(B^{T} B\right)^{1 / 2}$ is the volume of a fundamental domain.

Remark 2.1 Given $\Lambda=g \mathbb{Z}^{n}$ with $g \in \mathrm{GL}(n, \mathbb{R})$, then any $l$-dimensional sublattice $\Delta \subset \Lambda$ is spanned by $g n_{1}, \ldots, g n_{l}$, where $n_{i} \in \mathbb{Z}^{n}$ and $\operatorname{det}(\Delta)=$ $\left\|g n_{1} \wedge \ldots \wedge g n_{l}\right\|$. If $\Delta^{\prime} \subset \Lambda$ is a sublattice distinct from $\Delta$ with basis $g n_{1}^{\prime}, \ldots, g n_{l}^{\prime}, n_{i}^{\prime} \in \mathbb{Z}^{n}$, then

$$
\begin{aligned}
& \left\|\left(g n_{1} \wedge \cdots \wedge g n_{l}\right)-\left(g n_{1}^{\prime} \wedge \ldots \wedge g n_{l}^{\prime}\right)\right\| \\
& \quad \gg_{g}\left\|\left(n_{1} \wedge \ldots \wedge n_{l}\right)-\left(n_{1}^{\prime} \wedge \cdots \wedge n_{l}^{\prime}\right)\right\| \geq 1,
\end{aligned}
$$

since the $l$-th exterior product of $g$ is invertible. This argument shows that the $\alpha_{l}$-characteristic is attained at some $l$-dimensional sublattice $\Lambda^{\prime} \subset \Lambda$.

In the special case $n=2 d$ we also introduce

$$
\gamma_{\left[T_{-}, T\right], \beta}(r) \stackrel{\text { def }}{=} \sup \left\{\left(r^{-d} \alpha_{d}\left(\Lambda_{t}\right)\right)^{1 / 2-\beta}: T_{-} \leq|t| \leq T\right\},
$$

where $\Lambda_{t}=d_{r} u_{t} \Lambda_{Q}$ denotes a $2 d$-dimensional lattice obtained by an appropriate action of $d_{r}, u_{t} \in \operatorname{SL}(2, \mathbb{R})$ on $\mathbb{R}^{2 d}$ (see (4.25)), where $d_{r}$ and $u_{t}$ denote the usual diagonal and unipotent elements and $\Lambda_{Q}$ denotes a fixed $2 d$-dimensional lattice depending on $Q$ (see (4.28)). Recall that $E_{a, b}=\{x \in$ $\left.\mathbb{R}^{d}: a<Q[x]<b\right\}$ and let $\mathrm{v}(x)$ denote a smooth weight function such that $\zeta(x):=\mathrm{v}(x) \exp \left\{Q_{+}[x]\right\}$ satisfies

$$
\sup _{x \in \mathbb{R}^{d}}(|\zeta(x)|+|\widehat{\zeta}(x)|)(1+\|x\|)^{d+1}<\infty .
$$

An explicit construction of weight functions for parallelepiped regions will be given in Sect. 7. Nevertheless, as a simple example, one can take the Gaussian weights $\mathrm{v}(x)=\exp \left\{-2 Q_{+}[x]\right\}$.

Theorem 2.2 Let $Q$ be a non-degenerate quadratic form in $d \geq 5$ variables with $q_{0} \geq 1$. Choose $\beta=\frac{2}{d}+\frac{\delta}{d}$ for some arbitrary small $\delta \in\left(0, \frac{1}{10}\right)$. Write 
$(b-a)_{q}:=b-a$ if $b-a \leq q$ and $(b-a)_{q}:=q^{\beta d-1 / 2}$ if $b-a>q$, and $(b-a)^{*}:=(b-a)$ if $b-a \leq 1$ and $(b-a)^{*}:=1$ if $b-a>1$. Then for any $r \geq q^{1 / 2}, b>a$ and $0<\bar{w}<(b-a) / 4$ we have

$$
\begin{aligned}
\left|\sum_{m \in E_{a, b} \cap \mathbb{Z}^{d}} \mathrm{v}\left(\frac{m}{r}\right)-\int_{E_{a, b}} \mathrm{v}\left(\frac{x}{r}\right) \mathrm{d} x\right| \ll_{\beta, d}\left\{w\left\|_{\mathrm{v}}\right\|_{Q}\right. & \left.+\|\widehat{\zeta}\|_{1} C_{Q} \rho_{Q, b-a}^{w}(r)\right\} r^{d-2} \\
& +d_{Q} r^{d / 2}\|\hat{\zeta}\|_{*, r} \log \left(1+\frac{|b-a|}{q_{0}^{1 / 2} r}\right),
\end{aligned}
$$

where $C_{Q}:=q|\operatorname{det} Q|^{-1 / 4-\beta / 2}$ and $\|\mathrm{v}\|_{Q}$ is defined in Lemma 7.1 (the quantity $\|\mathrm{v}\|_{Q}$ depends additionally on $r, a, b$ and $w$, but we will suppress this dependence),

$$
\begin{aligned}
\rho_{Q, b-a}^{w}(r) \stackrel{\operatorname{def}}{=} \inf \left\{(b-a)_{q}\left(c_{Q} T_{-}^{\frac{d}{2}-2-\delta}+\gamma_{\left[T_{-}, 1\right], \beta}(r)\right)+\gamma_{\left(1, T_{+}\right], \beta}(r)\left(1+\log \left((b-a)^{*} T_{+}\right)\right)\right. \\
\left.+c_{Q}^{-1}\left(T_{+} w\right)^{-1 / 2} \mathrm{e}^{-\left(T_{+} w\right)^{1 / 2}}: T_{-} \in\left[q_{0}^{-1 / 2} r^{-1}, 1\right], T_{+} \geq 1\right\}
\end{aligned}
$$

and $c_{Q}:=|\operatorname{det} Q|^{1 / 4-\beta / 2}$. Furthermore

$$
\|\widehat{\zeta}\|_{*, r} \stackrel{\text { def }}{=} q^{d / 4}\left(\left(\frac{q}{q_{0}}\right)^{d / 2}\|\widehat{\zeta}\|_{1}+\int_{\|v\|_{\infty}>r / 2} \frac{|\widehat{\zeta}(v)|}{\left(q^{1 / 2} r^{-1}+\left\|v r^{-1}\right\|_{\mathbb{Z}}\right)^{d / 2}} \mathrm{~d} v\right)
$$

and here $\|v\|_{\mathbb{Z}}:=\min _{m \in \mathbb{Z}^{d}}\|v-m\|_{\infty}$.

We use the notation $A \asymp_{d} B$ for quantities of equivalent size up to constants depending on $d$ only, i.e. $A \ll_{d} B \ll_{d} A$.

\section{Remark 2.3 Note that}

a) Theorem 2.2 extends to affine quadratic forms $Q[x+\xi]$ uniformly in $|\xi|_{\infty} \leq 1$

b) Depending on the application, the lattice remainder (2.5) will be optimized in the parameters $w, \varepsilon$ and $T_{+}$differently: For thin shells the error should also scale with the length $b-a$. This forces $T_{+}$to be large and requires 'strong' Diophantine assumptions. In the case of wide shells it is possible to choose $w$ relatively large.

c) If $Q$ is irrational, then Corollary 4.11 implies that $\rho_{Q, b-a}^{w}(r) \rightarrow 0$ for $r \rightarrow \infty$, provided that $w$ and $(b-a)$ are fixed. The first factor in the definition of $\rho_{Q, b-a}^{w}$ corresponds to small values of $t$ on the Fourier side and the last factor to the decay rate of the $w$-smoothing of the interval $[a, b]$.

With these notations we state a result providing quantitative bounds for the difference between the volume and the lattice point volume in $E_{a, b}$. 


\subsection{Ellipsoids $E_{0, b}$}

Here $Q$ is positive definite and we may assume that $b$ tends to infinity. Let $r=\sqrt{2 b}$ in Theorem 2.2. Then the ellipsoid $E_{0, b}=\left\{x \in \mathbb{R}^{d}: Q[x] \leq b\right\}$ is contained in $r \Omega=Q_{+}^{-1 / 2}[-r, r]^{d}$. Choosing in Theorem 2.2 a smoothing of $I_{\Omega}$, say $\mathrm{v}_{\varepsilon}$ of width $\varepsilon=\frac{1}{15}$, which equals 1 on $E_{0, b}$, and the smoothing parameter $w$ in terms of $T_{+}$, such that the right-hand side in (2.5) is minimal, will lead to

Corollary 2.4 Let $Q$ denote a non-degenerate $d$-dimensional positive definite form with $d \geq 5$ and $q_{0} \geq 1$. For any $r \geq q^{1 / 2}$ and $r=\sqrt{2 b}$ we have with $H_{r}:=E_{0, b}$

$$
\left|\operatorname{vol}_{\mathbb{Z}} H_{r}-\operatorname{vol} H_{r}\right| \ll_{\beta, d} d_{Q} r^{d-2}\left(\rho_{Q}^{\mathrm{ell}}(r)+q^{d / 4} r^{-d / 2+2}\left(q / q_{0}\right)^{d / 2} \log (r)\right),
$$

where

$$
\rho_{Q}^{\text {ell }}(r) \stackrel{\text { def }}{=} \inf \left\{a_{Q}\left(q^{\frac{3}{2}+\delta}\left(c_{Q} T_{-}^{\frac{d}{2}-2-\delta}+\gamma_{\left[T_{-}, 1\right], \beta}(r)\right)+\gamma_{\left(1, T_{+}\right], \beta}(r) \log \left(T_{+}+1\right)\right)+\frac{\log \left(1+q T_{+}\right)^{2}}{T_{+}}\right\}
$$

and the infimum is taken over $T_{-} \in\left[q_{0}^{-1 / 2} r^{-1}, 1\right]$ and $T_{+} \geq 1$, where $a_{Q}=$ $q|\operatorname{det} Q|^{\frac{1}{4}-\frac{\beta}{2}}, c_{Q}=|\operatorname{det} Q|^{1 / 4-\beta / 2}$. Furthermore, $\lim _{r \rightarrow \infty} \rho_{Q}^{\text {ell }}(r)=0$ as $r$ tends to infinity, provided that $Q$ is irrational.

Compared to the quantitative results in $[5,6]$, this bound holds already for $d \geq 5$. Moreover, Corollary 2.4 refines the estimates obtained in [28].

\subsection{Hyperboloid shells $E_{a, b}$}

If $Q$ is indefinite, we distinguish, depending on $b-a$, between 'small' and 'wide' shells $E_{a, b}$. Here we restrict ourselves to a special class of rescaled admissible parallelepipeds $r \Omega$ for $r>0$ : We suppose that $\Omega=B^{-1}[-1,1]^{d}$ is determined by some $B \in \mathrm{GL}(d, \mathbb{R})$ such that the lattice $\Gamma=B \mathbb{Z}^{d}$ is admissible in the sense of Sect. 7.3, i.e. both (7.29) and (7.1) should be satisfied (for examples, see Remark 7.4 and Example 7.6). Note that the latter condition (7.1), that is $Q_{+} \leq B^{T} B \leq c_{B} Q_{+}$with $c_{B} \geq 1$, ensures that the region $\Omega$ is rescaled with respect to the quadratic form $Q$.

To estimate the lattice point remainder for this restriction of $E_{a, b}$ given by $H_{r}:=E_{a, b} \cap r \Omega$ we smooth the indicator function $I_{\Omega}$ in an $\varepsilon$-neighborhood with an error of order $\mathcal{O}\left(\varepsilon(b-a) r^{d-2}\right)$ using Lemma 7.1. This yields a smooth function $\mathrm{v}_{\varepsilon}$ and a final weight function $\zeta_{\varepsilon}$, according to (2.4) in Theorem 2.2. Since $\Omega$ is admissible, both $\left\|\zeta_{\varepsilon}\right\|_{1}$ and $\left\|\zeta_{\varepsilon}\right\|_{*, r}$ in (2.6) are growing with a power of $|\log \varepsilon|$ only, see Lemmas 7.2 and 7.8. 
In the next step we calibrate both smoothing parameters $w$ and $\varepsilon$ in order to get Corollary 2.5 below for 'wide' and 'thin' shells. The actual choice of $\varepsilon$ is then determined by calibrating the main terms $\varepsilon r^{d-2}$ and $\left\|\zeta_{\varepsilon}\right\|_{1} \rho_{Q, b-a}^{w}(r) r^{d-2}$ depending on the speed of convergence of $\lim _{r \rightarrow \infty} \rho_{Q, b-a}^{w}(r)=0$. The resulting error bound for indefinite forms will then differ at most by some $|\log \varepsilon|$-factors from the positive definite case, and is thus dominantly influenced by the Diophantine properties reflected in the decay of the $\gamma_{\left[T_{-}, T_{+}\right], \beta}$, resp. the $\rho_{Q, b-a}^{w}$-characteristic of irrationality. In particular we have uniformly for 'small' and 'wide' shells $E_{a, b}$ and admissible regions $\Omega$ the following bound:

Corollary 2.5 Under the assumptions of Theorem 2.2 we get for an admissible region $\Omega$, all $\max \{|a|,|b|\} \leq c_{0} r^{2}$, where $c_{0}>0$ is chosen as in Lemma 7.1, and $b-a \geq q$

$$
\Delta_{r} \stackrel{\text { def }}{=}\left|\operatorname{vol}_{\mathbb{Z}} H_{r}-\operatorname{vol} H_{r}\right| \ll_{\beta, d} d_{Q} r^{d-2}\left(\rho_{Q, b-a}^{\text {hyp }+}(r)+R_{Q, A}(r)\right),
$$

where

$$
R_{Q, A}(r) \stackrel{\text { def }}{=} q^{\frac{d}{4}} r^{-\frac{d}{2}+2} \log (r+1)^{d}\left(\left(\frac{q}{q_{0}}\right)^{\frac{d}{2}}+\frac{c_{B}^{d / 2} q_{0}^{-\frac{d}{4}}}{\mathrm{Nm}(\Gamma)} \log \left(2+\frac{1}{\mathrm{Nm}(\Gamma)}\right)\right) \log \left(1+\frac{b-a}{q_{0}^{1 / 2} r}\right),
$$

$\operatorname{Nm}(\Gamma):=\inf _{\gamma \in \Gamma \backslash\{0\}}\left|\gamma_{1} \ldots \gamma_{d}\right|$ in standard coordinates $\gamma=\left(\gamma_{1}, \ldots, \gamma_{d}\right)$ and

$$
\begin{array}{r}
\rho_{Q, b-a}^{\text {hyp }+}(r) \stackrel{\text { def }}{=} \inf _{T_{+}, T_{-}}^{*}\left\{\operatorname { l o g } ( ( b - a ) T _ { - } ^ { - ( \frac { d } { 2 } - 2 - \delta ) } + 1 ) ^ { d } \left(a_{Q} q^{\frac{3}{2}+\delta}\left(c_{Q} T_{-}^{\frac{d}{2}-2-\delta}+\gamma_{\left[T_{-}, 1\right], \beta}(r)\right)\right.\right. \\
\left.\left.+a_{Q} \gamma_{\left(1, T_{+}\right], \beta}(r) \log \left(T_{+}+1\right)+\frac{\log \left(q T_{+}+1\right)^{2}}{T_{+}}\right)\right\},
\end{array}
$$

where the infimum is taken over all $T_{-} \in\left[q_{0}^{-1 / 2} r^{-1}, 1\right]$ and $T_{+} \geq 1$. If $b-a \leq q$, then (2.8) holds, too, whereby the Diophantine factor $\rho_{Q, b-a}^{\text {hyp }+}(r)$ has to be replaced by

$$
\begin{array}{r}
\rho_{Q, b-a}^{\text {hyp }-}(r) \stackrel{\text { def }}{=} \inf _{T_{-}, T_{+}}^{*}\left\{a _ { Q } \operatorname { l o g } ( 1 + T _ { - } ^ { - ( \frac { d - 4 } { 2 } - \delta ) } ) ^ { d } \left((b-a)\left(c_{Q} T_{-}^{\frac{d}{2}-2-\delta}+\gamma_{\left[T_{-}, 1\right], \beta}(r)\right)\right.\right. \\
\left.\left.+\gamma_{\left(1, T_{+}\right], \beta}(r)\left(\log \left((b-a)^{*} T_{+}\right)+1\right)\right)\right\} .
\end{array}
$$

In the last equation the infimum is taken over all $T_{-} \in\left[q_{0}^{-1 / 2} r^{-1}, 1\right]$ and $T_{+} \geq 1$ with

$$
T_{+} \geq 4(b-a)^{-1} T_{-}^{-\left(\frac{d}{2}-2-\delta\right)} \max \left\{1, \log \left(c_{Q}^{2}(b-a) T_{-}^{\frac{d}{2}-2-\delta}\right)^{2}\right\}
$$


These bounds refine the results obtained in [6] providing explicit estimates in terms of $Q$ and are valid for $d \geq 5$. Note that, due to the 'uncertainty principle' for the Fourier transform, we need to choose $T_{+}$at least as large as in (2.10) if $E_{a, b}$ is 'thin' in order to control the factor $\exp \left\{-\left|T_{+} w\right|^{1 / 2}\right\}$ (occurring in the definition of $\rho_{Q, b-a}^{w}$ ) which scales with $b-a$. In Sect. 7.4 we prove a variant of Corollary 2.5 for thin shells and non-admissible regions $\Omega$ as well, see Corollary 7.10.

\subsection{Quadratic forms of diophantine type $(\kappa, A)$}

For any fixed $T_{+}>1>T_{-}>0$ and irrational $Q$ it is shown in Corollary 4.11 that

$$
\lim _{r \rightarrow \infty} \gamma_{\left[T_{-}, T_{+}\right], \beta}(r)=0,
$$

with a speed depending on the Diophantine properties of $Q$. For indefinite forms $Q$, this implies for fixed $b-a>0$ that

$$
\lim _{r \rightarrow \infty} \rho_{Q, b-a}^{\text {hyp }+}(r)=0, \quad \lim _{r \rightarrow \infty} \rho_{Q, b-a}^{\text {hyp }-}(r)=0
$$

and hence $\Delta_{r}=o\left(r^{d-2}\right)$ as $r \rightarrow \infty$. This holds uniformly for all intervals $[a, b]$ with $0<u_{r} \leq b-a \leq v_{r} \leq c_{0} r^{2}$ and sequences $\lim _{r} u_{r}=0$, $\lim _{r} v_{r}=\infty, r \rightarrow \infty$ depending on $Q$. For the special class of quadratic forms of Diophantine type $(\kappa, A)$, as introduced in Definition 1.6, we may apply Corollary 4.11 to obtain explicit bounds on the Diophantine factors in the previous theorems as follows.

Corollary 2.6 Consider an indefinite quadratic form $Q$ that is Diophantine of type $(\kappa, A)$. Moreover, let $\beta=2 / d+\delta / d$ for some sufficiently small $0<$ $\delta<\frac{1}{10}$. Then for the case of wide shells $b-a \geq q$ in Corollary 2.5 we have

$$
\rho_{Q, b-a}^{\text {hyp }+}(r) \ll_{\beta, d} \log (r+1)^{d} h_{Q} q^{\frac{3}{2}+v+\delta}\left(1+A^{-v}\right)\left(r^{-\frac{d-2(2+\delta)}{d(k+1)+1}}+r^{-\frac{2 v}{k v+1}} \log (q r+1)\right),
$$

where $h_{Q}=q|\operatorname{det} Q|^{1 / 2-\beta}, v=(1-2 \beta) /(2 \kappa+2)$ and $\sigma=d(1 / 2-\beta)$. Thus for an admissible region $\Omega$ satisfying (7.1) we have for all $r \geq q^{1 / 2}$ and $\max \{|a|,|b|\} \leq c_{0} r^{2}$

$$
\left|\frac{\mathrm{vol}_{\mathbb{Z}} H_{r}}{\operatorname{vol} H_{r}}-1\right| \ll_{Q, \Omega, \beta, d} \frac{\log (r+1)^{d}}{b-a}\left(r^{-\frac{(1-2 \beta) d}{1+(\kappa+1) d}}+r^{-\frac{2-4 \beta}{2+(3-2 \beta) \kappa}}+r^{-\frac{d}{2}+2} \log \left(1+\frac{b-a}{r}\right)\right),
$$


where the implied constant in (2.14) can be explicitly determined. For thin shells, i.e. $b-a \leq q$, we have

$$
\begin{array}{r}
\rho_{Q, b-a}^{\text {hyp }-}(r) \ll \beta, d \\
\inf _{T_{-}, T_{+}}^{*}\left\{h _ { Q } \operatorname { l o g } ( 1 + T _ { - } ^ { - ( \frac { d } { 2 } - 2 - \delta ) } ) ^ { d } \left((b-a)\left(T_{-}^{\frac{d}{2}-2-\delta}+q^{v} A^{-v} T_{-}^{-v} r^{-2 v}\right)\right.\right. \\
\left.\left.\left.+q^{v} A^{-v} T_{+}^{k v} r^{-2 v}\left(\log \left((b-a)^{*} T_{+}\right)\right)+1\right)\right)\right\},
\end{array}
$$

where the infimum is taken over all $T_{-} \in\left[q_{0}^{-1 / 2} r^{-1}, 1\right]$ and $T_{+} \geq 1$ restricted to

$$
T_{+} \geq 4(b-a)^{-1} T_{-}^{-\left(\frac{d}{2}-2-\delta\right)} \max \left\{1, \log \left(c_{Q}^{2}(b-a) T_{-}^{-\left(\frac{d}{2}-2-\delta\right)}\right)^{2}\right\} .
$$

\section{Fourier analysis}

\subsection{Smoothing}

The first step in the proof of Theorem 2.2 is to rewrite the lattice point counting error (i.e. the left hand side of (2.5)) in terms of integrals over appropriate smooth functions. To this end, we introduce smooth approximation of the indicator functions of $E_{a, b}$ and $\Omega$ constructed as follows. Denote by $k=k(x) \mathrm{d} x$ a probability measure (symmetric around 0 ) with compact support satisfying $k([-1,1])=1$ and $|\widehat{k}(t)| \leq C \exp \left\{-|t|^{1 / 2}\right\}$ for all $t \in \mathbb{R}$ and a positive constant $C>0$, where $\widehat{k}(t):=\int k(x) \exp \{-2 \pi \mathrm{i} t x\} \mathrm{d} x$ denotes the Fourier transform of the measure $k$. For an example of $k$ we refer to Corollary 10.4 in [10]. More generally, by a result of Ingham [31] (see e.g. Theorem 10.2 in [10]) there is a probability density $k$ such that $|\widehat{k}(t)| \leq C \exp \{-u(|t|)|t|\}$, where $u$ is a continuous, non-negative, non-increasing function on $[0, \infty)$ satisfying $\int_{1}^{\infty} u(t) t^{-1} \mathrm{~d} t<\infty$ and this condition is also necessary. However, we will not need this improved decay rate. For $\tau>0$ let $k_{\tau}$ denote the rescaled measures $k_{\tau}(A):=k\left(\tau^{-1} A\right)$ for any $A \in \mathcal{B}^{d}$, where $\mathcal{B}^{d}$ denotes the Borel $\sigma$-algebra. Using the same notation, let $k_{\tau}(x)=k_{\tau}\left(x_{1}\right) \ldots k_{\tau}\left(x_{d}\right), x=\left(x_{1}, \ldots, x_{d}\right)$, denote its multivariate extension on $\mathbb{R}^{d}, d \geq 1$. Furthermore, let $f * k_{\tau}$ denote the convolution of a function $f$ on $\mathbb{R}^{d}$ and $k_{\tau}$. We need the following standard estimate for smooth approximations.

Lemma 3.1 Let $\mu$ and $\nu$ be (positive) finite measures on $\mathbb{R}^{d}$, let $f$ and $f_{\tau}^{ \pm}, \tau>$ 0 , denote bounded real-valued Borel-measurable functions on $\mathbb{R}^{d}$ such that for any $\tau>0$

$$
\begin{array}{ll}
f_{\tau}^{-}(x) \leq \inf \left\{f(y):\|y-x\|_{\infty}<\tau\right\} & \text { and } \quad f_{\tau}^{+}(x) \geq \sup \left\{f(y):\|y-x\|_{\infty}<\tau\right\}, \\
f_{2 \tau}^{-}(x) \leq \inf \left\{f_{\tau}^{-}(y):\|y-x\|_{\infty}<\tau\right\} & \text { and } \quad f_{2 \tau}^{+}(x) \geq \sup \left\{f_{\tau}^{+}(y):\|y-x\|_{\infty}<\tau\right\} .
\end{array}
$$


Then

$$
\left|\int f \mathrm{~d}(\mu-v)\right| \leq \max _{ \pm}\left|\int f_{\tau}^{ \pm} \mathrm{d}(\mu-v) * k_{\tau}\right|+\int\left(f_{2 \tau}^{+}-f_{2 \tau}^{-}\right) \mathrm{d} \nu
$$

Proof Note that $k_{\tau}$ is a probability measure with support contained in a $\|\cdot\|_{\infty^{-}}$ ball of radius $\tau$. Hence, (3.1) implies the following chain of inequalities

$$
f_{2 \tau}^{-} \leq f_{\tau}^{-} * k_{\tau} \leq f \leq f_{\tau}^{+} * k_{\tau} \leq f_{2 \tau}^{+},
$$

which leads to

$$
\int f \mathrm{~d}(\mu-v) \leq \int f_{\tau}^{+} * k_{\tau} \mathrm{d}(\mu-v)+\int\left(f_{\tau}^{+} * k_{\tau}-f\right) \mathrm{d} v
$$

together with a similar lower bound. Since by (3.3) $f \leq f_{\tau}^{+} * k_{\tau} \leq f_{2 \tau}^{+}$and $f \geq f_{\tau}^{-} * k_{\tau} \geq f_{2 \tau}^{-}$, the upper bound (3.4) together with the corresponding lower bound proves the lemma.

First we shall investigate approximations to the sum under consideration, counting the lattice points in $E_{a, b}$ with weights $\mathrm{v}_{r}(x):=\mathrm{v}(x / r)$. In accordance with the notation introduced in (1.15) at the beginning of Sect. 1.3.1, we write

$$
\sum_{m \in \mathbb{Z}^{d}} I_{[a, b]}(Q[m]) \mathrm{v}_{r}(m)=\int_{\mathbb{R}^{d}} I_{[a, b]}(Q[x]) \mathrm{v}_{r}(x) \mathrm{d} x+R\left(I_{E_{a, b}} \mathrm{v}_{r}\right),
$$

where $\mathrm{v}(x)$ is a sufficiently fast decreasing smooth function such that the function

$$
\zeta(x) \stackrel{\text { def }}{=} \mathrm{v}(x) \exp \left\{Q_{+}[x]\right\}
$$

satisfies (2.4). For such weights both sides of (3.5) are well defined and $R\left(I_{E_{a, b}} \mathrm{v}_{r}\right)$ may be estimated by Poisson's formula, see [8], §46. By means of Lemma 3.1 we now replace the indicator $I_{[a, b]}$ by a smooth approximation.

Corollary 3.2 Let $[a, b]_{\tau}:=[a-\tau, b+\tau]$ and write

$$
g_{ \pm w} \stackrel{\text { def }}{=} I_{[a, b]_{ \pm w}} * k_{w} \text { and } g_{ \pm w}^{Q}(x) \stackrel{\text { def }}{=} g_{ \pm w}(Q[x]), \quad x \in \mathbb{R}^{d},
$$

where $0<w<(b-a) / 4$. Then

$$
\left|R\left(I_{E_{a, b}} \mathrm{v}_{r}\right)\right| \leq \max _{ \pm}\left|R\left(g_{ \pm w}^{Q} \mathrm{v}_{r}\right)\right|+c_{d} w\|\mathrm{v}\|{ }_{Q} r^{d-2},
$$


where $R\left(g_{ \pm w}^{Q} \mathrm{v}_{r}\right)$ is defined in accordance to (3.5), $\|\mathrm{v}\|_{Q}$ is defined in Lemma 7.1 and $c_{d}$ is a positive constant depending on $d$ only.

Proof In Lemma 3.1 we choose the measure $\mu$, resp. $v$, on $\mathbb{R}$ as the induced measure under the map $x \mapsto Q[x]$ of the counting measure with weights $\mathrm{v}_{r}(m)$, resp. the measure $\mathrm{v}_{r}(x) \mathrm{d} x$. Let $f(z)=I_{[a, b]}(z)$ and $f_{\tau}^{ \pm}(z)=$ $I_{[a, b]_{ \pm \tau}}(z)$. Then (3.1) is satisfied and (3.2) applies with $\tau=w$. In order to bound the remainder term in (3.2) observe that

$$
f_{2 w}^{+}-f_{2 w}^{-} \leq I\left(\left\{x \in \mathbb{R}^{d}: Q[x] \in[a-2 w, a+2 w] \cup[b-2 w, b+2 w]\right\}\right)
$$

and apply the geometric estimate of Lemma 7.1; that is (7.10) of Sect. 7.1.

Thus we have reduced the determination of the lattice point remainder $R\left(I_{E_{a, b}} \mathrm{v}_{r}\right)$ to the remainder $R\left(g_{ \pm w}^{Q} \mathrm{v}_{r}\right)$ for smooth weights. In the next subsection we shall rewrite the latter by means of the corresponding Fourier transforms.

\subsection{Fourier transforms and theta-series}

Rewrite the weight factor $\mathrm{v}$ in (3.5) as $\mathrm{v}(x)=\exp \left\{-Q_{+}[x]\right\} \zeta(x)$. Since by definition (see the previous Sect. 3.1)

$$
\left|\widehat{g}_{ \pm w}(t)\right| \ll\left|\widehat{I}_{[a, b]_{ \pm w}}(t) \widehat{k}_{w}(t)\right| \ll s_{[a, b]_{ \pm w}}(t) \exp \left\{-|t w|^{1 / 2}\right\} \text { and } \widehat{\zeta} \in L^{1}(\mathrm{~d} v)
$$

where

$$
s_{[a, b]_{ \pm w}}(t) \stackrel{\text { def }}{=}\left|(2 \pi t)^{-1} \sin (\pi t(b-a \pm 2 w))\right|,
$$

we may express the weight functions $g_{ \pm w}$ and $\zeta$ by their Fourier transforms

$$
\begin{aligned}
\widehat{g}_{ \pm w}(v) & =\int_{\mathbb{R}} g_{ \pm w}(x) \exp \{-2 \pi \mathrm{i} t x\} \mathrm{d} x \text { and } \\
\widehat{\zeta}(v) & =\int_{\mathbb{R}^{d}} \zeta(x) \exp \{-2 \pi \mathrm{i}\langle v, x\rangle\} \mathrm{d} x .
\end{aligned}
$$

This yields

$$
\begin{aligned}
g_{ \pm w}(Q[x]) & =\int_{\mathbb{R}^{\prime}} \widehat{g}_{ \pm w}(t) \exp \{2 \pi \mathrm{i} t Q[x]\} \mathrm{d} t \\
\zeta(x) & =\int_{\mathbb{R}^{d}} \widehat{\zeta}(v) \exp \{2 \pi \mathrm{i}\langle x, v\rangle\} \mathrm{d} v .
\end{aligned}
$$


Using (3.10) we obtain by interchanging summation and integration in (3.5)

$$
R\left(g_{ \pm w}^{Q} \mathrm{v}_{r}\right)=\int_{\mathbb{R}} R\left(e_{t} Q \mathrm{v}_{r}\right) \widehat{g}_{ \pm w}(t) \mathrm{d} t
$$

with $e_{t}(x):=\exp \{2 \pi \mathrm{i} t Q[x]\}$. (Here $R\left(e_{t} Q_{\mathrm{v}_{r}}\right)$ denotes the inner integral with respect to the variable $v$.) In the same way, writing $\tilde{e}_{v, r}(x):=$ $\exp \left\{-Q_{+}[x / r]+2 \pi \mathrm{i}\left\langle x, v r^{-1}\right\rangle\right\}$, we derive by (3.11) the remainder

$$
R\left(e_{t} \mathrm{v}_{r}\right)=\int_{\mathbb{R}^{d}} R\left(e_{t} \tilde{e}_{v, r}\right) \widehat{\zeta}(v) \mathrm{d} v .
$$

The sum $R\left(e_{t} Q \tilde{e}_{v, r}\right)$ is the remainder between the generalized theta series and its corresponding theta integral, that is $R\left(e_{t Q} \tilde{e}_{v, r}\right)=\theta_{v}(z)-\vartheta_{v}(t)$, where

$$
\begin{aligned}
& \theta_{v}(t) \stackrel{\text { def }}{=} \sum_{x \in \mathbb{Z}^{d}} \exp \left\{Q_{r, v}(t, x)\right\} \text { and } \vartheta_{v}(t) \stackrel{\text { def }}{=} \int_{\mathbb{R}^{d}} \exp \left\{Q_{r, v}(t, x)\right\} \mathrm{d} x, \\
& Q_{r, v}(t, x) \stackrel{\text { def }}{=} 2 \pi \mathrm{i} t Q[x]-r^{-2} Q_{+}[x]+2 \pi \mathrm{i}\left\langle x, v r^{-1}\right\rangle .
\end{aligned}
$$

Let us note that both $\vartheta_{t}(v)$ as well as $\theta_{t}(v)$ depend on the dilating variable $r$. However, we shall suppress this underlying dependency in order to reduce the notational burden. For $|t| \leq q_{0}^{-1 / 2} r^{-1}$ we shall use following representations of $R\left(e_{t} Q \tilde{e}_{v, r}\right)=\theta_{v}(z)-\vartheta_{v}(t)$ in (3.12) by means of Poisson's formula (see [8], §46), which obviously applies here:

$$
\theta_{v}(t)-\vartheta_{v}(t)=\sum_{m \in \mathbb{Z}^{d} \backslash\{0\}} \vartheta_{v-r m}(t)
$$

Note that by definition (3.14) the Fourier transform of $x \mapsto \exp \left\{Q_{r, v}(t, x)\right\}$ at $u \in \mathbb{R}^{d}$ is given by $\vartheta_{v-r u}(t)$, where

$$
\begin{gathered}
\exp \left\{Q_{r, v}(t, x)\right\}=\exp \left\{-\tilde{Q}_{t}[x]+2 \pi \mathrm{i}\left\langle x, v r^{-1}\right\rangle\right\}, \\
\tilde{Q}_{t} \stackrel{\text { def }}{=} r^{-2} Q_{+}-2 \pi \mathrm{i} t Q .
\end{gathered}
$$

In view of (3.13) and (3.16) we have

$$
R\left(e_{t} \mathrm{v}_{r}\right)=\int_{\mathbb{R}^{d}}\left(\sum_{m \in \mathbb{Z}^{d} \backslash\{0\}} \vartheta_{v-r m}(t)\right) \widehat{\zeta}(v) \mathrm{d} v .
$$

From here we only consider the weight $g_{w}$. The same inequalities hold also for $g_{w}$ replaced with $g_{-w}$. Next, we decompose the integral over $t$ in (3.12) 
into the segments $J_{0}:=\left[-q_{0}^{-1 / 2} r^{-1}, q_{0}^{-1 / 2} r^{-1}\right]$ and $J_{1}:=\mathbb{R} \backslash J_{0}$ and obtain

$$
\left|R\left(g_{w}^{Q} \mathrm{v}_{r}\right)\right| \ll_{d} I_{\Delta}+I_{\vartheta}+I_{\theta},
$$

where,

$$
\begin{aligned}
& I_{\Delta} \stackrel{\text { def }}{=}\left|\int_{J_{0}} R\left(e_{t} Q \mathrm{v}_{r}\right) \widehat{g}_{w}(t) \mathrm{d} t\right|, \\
& I_{\vartheta} \stackrel{\text { def }}{=}\left|\int_{J_{1}} \widehat{g}_{w}(t) \int_{\mathbb{R}^{d}} \vartheta_{v}(t) \widehat{\zeta}(v) \mathrm{d} v \mathrm{~d} t\right|, \\
& I_{\theta} \stackrel{\text { def }}{=}\left|\int_{J_{1}} \widehat{g}_{w}(t) \int_{\mathbb{R}^{d}} \theta_{v}(t) \widehat{\zeta}(v) \mathrm{d} v \mathrm{~d} t\right| .
\end{aligned}
$$

We start with the integral over the sections $J_{1}$. In the term $I_{\theta}$ we separate the $t$ and $v$ integrals via

$$
I_{\theta} \ll_{d}\|\widehat{\zeta}\|_{1} \sup _{v \in \mathbb{R}^{d}} \int_{|t|>q_{0}^{-1 / 2} r^{-1}}\left|\widehat{g}_{w}(t) \theta_{v}(t)\right| \mathrm{d} t
$$

where the estimation of the latter integral will be done in the Sects. 4-6. In order to estimate the terms $I_{\Delta}$ and $I_{\vartheta}$ we need to estimate $\left|\vartheta_{v}(t)\right|$ first:

\subsubsection{Estimates for $\left|\vartheta_{v}(t)\right|$}

For any symmetric complex $d \times d$-matrix $\Xi$, whose imaginary part is positive definite, we have

$$
\int_{\mathbb{R}^{d}} \exp \{\pi \mathrm{i} \Xi[x]+2 \pi \mathrm{i}\langle x, v\rangle\} \mathrm{d} x=(\operatorname{det}(\Xi / \mathrm{i}))^{-1 / 2} \exp \left\{-\pi \mathrm{i} \Xi^{-1}[v]\right\},
$$

where we choose the branch of the square root which takes positive values on purely imaginary $\Xi, v \in \mathbb{R}^{d}$ and $\Xi^{-1}[x]$ denotes the quadratic form $\left\langle\Xi^{-1} x, x\right\rangle$, defined by the inverse operator $\Xi^{-1}: \mathbb{C}^{d} \rightarrow \mathbb{C}^{d}$ whose imaginary part is negative definite (see [46], p. 195, Lemma 5.8 and (5.6)). We shall apply (3.24) in the case $\Xi_{t}:=\mathrm{i} \pi^{-1} \tilde{Q}_{t}=2 t Q+\mathrm{i} \pi^{-1} r^{-2} Q_{+}$in order to obtain the following expression for $\vartheta_{v}$ in (3.14) (see also (3.17))

$$
\vartheta_{v}(t)=\int_{\mathbb{R}^{d}} \exp \left\{\pi \mathrm{i} \Xi_{t}[x]+2 \pi \mathrm{i}\langle x, v / r\rangle\right\} \mathrm{d} x=\left(\operatorname{det}\left(\Xi_{t} / \mathrm{i}\right)\right)^{-\frac{1}{2}} \exp \left\{-\pi \mathrm{i} \Xi_{t}^{-1}[v / r]\right\} .
$$


Hence, the Fourier transform of $\left.x \mapsto \exp \left\{Q_{r, v}(t, x)\right]\right\}$ takes the following shape

$$
\operatorname{det}\left(\pi^{-1} \tilde{Q}_{t}\right)^{-1 / 2} \exp \left\{-\pi^{2} \tilde{Q}_{t}^{-1}[u-v / r]\right\}=\vartheta_{v-r u}(t)=\vartheta_{r u-v}(t) .
$$

A short calculation shows that $\tilde{Q}_{t}^{-1}=\left(4 \pi^{2} t^{2}+r^{-4}\right)^{-1}\left(2 \pi \mathrm{i} t Q^{-1}+r^{-2} Q_{+}^{-1}\right)$ and it follows immediately that

$$
\operatorname{det} \tilde{Q}_{t}^{-1}=\left(4 \pi^{2} t^{2}+r^{-4}\right)^{-d} \prod_{i=1}^{d}\left(2 \pi \mathrm{i} t q_{i}^{-1}+r^{-2}\left|q_{i}\right|^{-1}\right) .
$$

Taking the absolute value of (3.25) and (3.27) we conclude that

$$
\left|\vartheta_{u r}(t)\right| \ll_{d} d_{Q} r^{d / 2} r_{t}^{d / 2} \exp \left\{-\pi^{2} r_{t}^{2} Q_{+}^{-1}[u]\right\},
$$

where $r_{t}:=r\left(4 \pi^{2} t^{2} r^{4}+1\right)^{-1 / 2}$ and $d_{Q}:=|\operatorname{det} Q|^{-1 / 2}$ as already defined in (2.1). ${ }^{1}$

\subsubsection{Estimation of $I_{\vartheta}$}

By (3.28) with $v=u r$ we have $\left|\vartheta_{v}(t)\right| \ll_{d} d_{Q} r^{d / 2} r_{t}^{d / 2}$ and therefore we obtain by using (3.8) after integrating over $v$ in (3.21)

$$
I_{\vartheta} \ll_{d} d_{Q} r^{d / 2}\|\widehat{\zeta}\|_{1} \int_{|t|>q_{0}^{-1 / 2} r^{-1}} s_{[a, b]_{\omega}}(t) \exp \left\{-|w t|^{1 / 2}\right\} r_{t}^{d / 2} \mathrm{~d} t
$$

If $|b-a|^{-1} \leq q_{0}^{-1 / 2} r^{-1}$, then we use $s_{[a, b]_{w}}(t) \leq|t|^{-1}$ and $r_{t} \leq(r t)^{-1}$ to get the bound

$$
\int_{q_{0}^{-1 / 2} r^{-1}}^{\infty} s_{[a, b]_{w}}(t) r_{t}^{d / 2} \mathrm{~d} t \leq r^{-d / 2} \int_{q_{0}^{-1 / 2} r^{-1}}^{\infty} t^{-d / 2-1} \mathrm{~d} t \ll_{d} q_{0}^{d / 4} .
$$

In the case $|b-a|^{-1}>q_{0}^{-1 / 2} r^{-1}$ we shall estimate the $t$-integral in (3.29) by means of $s_{[a, b]_{w}}(t) \leq|b-a+2 w| / 2$. Using $|w|<(b-a) / 4$ additionally leads to

$$
\int_{|t|>q_{0}^{-1 / 2} r^{-1}} s_{[a, b] \omega}(t) r_{t}^{d / 2} \mathrm{~d} t \leq r^{-d / 2}|b-a+2 w| \int_{q_{0}^{-1 / 2} r^{-1}}^{\infty} t^{-d / 2} \mathrm{~d} t \ll_{d} \frac{|b-a|}{q_{0}^{1 / 2} r} q_{0}^{d / 4}
$$

$\overline{1}$ The first of these notations will be used throughout this section only and should not be confused with the notation $r_{*}:=r q^{-1 / 2}$ which will be introduced latter in Lemma 5.1. 
Summarizing, we have established the bound

$$
I_{\vartheta} \ll_{d} d_{Q}\|\widehat{\zeta}\|_{1} \min \left\{|b-a| q_{0}^{-1 / 2} r^{-1}, 1\right\} r^{d / 2} q_{0}^{d / 4},
$$

provided that $d>2$.

\subsubsection{Estimation of $I_{\Delta}$}

According to (3.20), (3.13) and (3.16) we may write

$$
\begin{aligned}
& I_{\Delta}=\left|\int_{J_{0}} \widehat{g}_{w}(t) R\left(e_{t} Q^{\mathrm{v}_{r}}\right) \mathrm{d} t\right|, \quad \text { where } \\
& R\left(e_{t} Q^{\mathrm{v}_{r}}\right)=\int_{\mathbb{R}^{d}} S_{t, v} \widehat{\zeta}(v) \mathrm{d} v, \quad S_{t, v} \stackrel{\text { def }}{=} \sum_{m \in \mathbb{Z}^{d} \backslash\{0\}} \vartheta_{v-r m}(t) .
\end{aligned}
$$

In order to use the estimate (3.28) let $v \in \mathbb{R}^{d}$ and write $v=r u$ with $u=$ $u_{0}+m_{u}$, where $u_{0} \in[-1 / 2,1 / 2]^{d}$ and $m_{u} \in \mathbb{Z}^{d}$. Then

$$
\left|S_{t, v}\right| \leq \sum_{m \neq m_{u}}\left|\vartheta_{r\left(u_{0}+m\right)}(t)\right| \ll d_{Q} r^{d / 2} r_{t}^{d / 2} \sum_{m \neq m_{u}} \exp \left\{-\pi^{2} r_{t}^{2} Q_{+}^{-1}\left[u_{0}+m\right]\right\}
$$

Note that $\left\|m+u_{0}\right\| \geq\left\|m+u_{0}\right\|_{\infty} \geq \frac{1}{2}$ for any $m \in \mathbb{Z}^{d} \backslash\{0\}$ and therefore $\frac{\pi^{2}}{2} Q_{+}^{-1}\left[u_{0}+m\right] \geq \frac{\pi^{2}}{8} q^{-1} \geq q^{-1}$ which yields the bound

$$
\left|S_{t, v}\right| \ll d_{Q} r^{d / 2}\left|r_{t}\right|^{d / 2}\left(\mathrm{e}^{-\pi^{2} r_{t}^{2} Q_{+}^{-1}\left[u_{0}\right]} I_{r}(v)+\mathrm{e}^{-r_{t}^{2} / q} K_{u_{0}}\right),
$$

where $I_{r}(v):=I_{[r / 2, \infty)}\left(\|v\|_{\infty}\right)$ and $K_{u_{0}}:=\sum_{m \in \mathbb{Z}^{d}} \exp \left\{-\frac{\pi^{2}}{2} r_{t}^{2} Q_{+}^{-1}[m+\right.$ $\left.\left.u_{0}\right]\right\}$. The sum $K_{u_{0}}$ may be estimated by an integral as follows: Since the map $t \mapsto r_{t}^{2}=r\left(4 \pi^{2} t^{2} r^{4}+1\right)^{-1 / 2}$ is strictly monotone increasing on $t<0$ and decreasing on $t>0$, we find that $r_{t}^{2} \geq q_{0} /\left(4 \pi^{2}+1\right)$ for $|t| \leq q_{0}^{-1 / 2} r^{-1}$ as $r \geq q^{\frac{1}{2}}$ and thus $\exp \left\{-\pi^{2} r_{t}^{2} Q_{+}^{-1}[u]\right\} \leq \exp \left\{-\frac{q_{0}}{5} Q_{+}^{-1}[u]\right\}$. Let $I:=\left[-\frac{1}{2}, \frac{1}{2}\right]^{d}$ and note that $Q_{+}^{-1}[x] \leq \frac{d}{4 q_{0}}$ for $x \in I$, from which we deduce that $k_{u} \stackrel{\text { def }}{=} \int_{I} \exp \left\{-\frac{q_{0}}{5} Q_{+}^{-1}[u+x]\right\} \mathrm{d} x \gg_{d} \exp \left\{-\frac{q_{0}}{5} Q_{+}^{-1}[u]\right\} \int_{I} \exp \left\{-\frac{2 q_{0}}{5}\left\langle Q_{+}^{-1} u, x\right\rangle\right\} \mathrm{d} x$,

where the integral on the right-hand side is at least one by Jensen's inequality. Hence

$$
K_{u_{0}} \leq \sum_{m \in \mathbb{Z}^{d}} \mathrm{e}^{-\frac{q_{0}}{5} Q_{+}^{-1}\left[m+u_{0}\right]} \ll_{d} \sum_{m \in \mathbb{Z}^{d}} k_{m+u_{0}}=\int_{\mathbb{R}^{d}} \mathrm{e}^{-\frac{q_{0}}{5} Q_{+}^{-1}[x]} \mathrm{d} x \ll_{d}\left(\frac{q}{q_{0}}\right)^{\frac{d}{2}} .
$$


Using (3.31) together with (3.33) and (3.34), we may now estimate $I_{\Delta}$ by the following integrals. Writing $v_{0}=v-r m,\left\|v_{0}\right\|_{\infty} \leq \frac{r}{2}, m \in \mathbb{Z}^{d}$, we have

$$
I_{\Delta} \ll_{d} d_{Q} \int_{J_{0}}\left|\widehat{g}_{w}(t)\right|\left(\Theta_{t, 1}+\Theta_{t, 2}\right) \mathrm{d} t,
$$

where

$$
\begin{aligned}
& \Theta_{t, 1} \stackrel{\text { def }}{=}\left(\frac{q}{q_{0}}\right)^{d / 2} r^{d / 2} r_{t}^{d / 2} \mathrm{e}^{-\frac{r_{t}^{2}}{q}} \int_{\mathbb{R}^{d}}|\widehat{\zeta}(v)| \mathrm{d} v \\
& \Theta_{t, 2} \stackrel{\text { def }}{=} r^{d / 2} r_{t}^{d / 2} \int_{\|v\|_{\infty}>r / 2} \exp \left\{-\pi^{2} r_{t}^{2} Q_{+}^{-1}\left[v_{0} r^{-1}\right]\right\}|\widehat{\zeta}(v)| \mathrm{d} v .
\end{aligned}
$$

If we write $h(s ; x):=s^{d / 4} \mathrm{e}^{-s x}$ with $s, x>0$, then the maximum of $s \mapsto h(s ; x)$ is attained at $s_{0}=d /(4 x)$. Hence, $\max _{t \in J_{0}} h\left(r_{t}^{2} ; x\right) \ll_{d}$ $\min \left(x^{-d / 4}, r^{d / 2}\right) \ll_{d}\left(x+\frac{1}{r^{2}}\right)^{-d / 4}$. Thus, we obtain with $x=1 / q$

$$
\max _{t \in J_{0}} \Theta_{t, 1} \ll_{d}\left(q / q_{0}\right)^{d / 2} r^{d / 2} q^{d / 4}\|\widehat{\zeta}\|_{1} .
$$

Note that the value $x=1 / q$ is within the range of $t \mapsto r_{t}^{2}, t \in J_{0}$, since its maximum is $r_{0}^{2}=r^{2}$ and its minimum is $q_{0} /\left(4 \pi^{2}+1\right) \leq r_{t^{*}}^{2} \leq q_{0}$, where $t^{*}= \pm q_{0}^{-1 / 2} r^{-1}$. In order to estimate $\Theta_{t, 2}$, we choose $x=Q_{+}^{-1}\left[v_{0} / r\right] / 4$ and get

$$
\sup _{t \in J_{0}} \Theta_{t, 2} \ll_{d} r^{d / 2} \int_{\|v\|_{\infty}>r / 2} \frac{\widehat{\zeta}(v) \mid}{\left(r^{-2}+Q_{+}^{-1}\left[v_{0} / r\right]\right)^{d / 4}} \mathrm{~d} v .
$$

Now we integrate the bounds (3.36) and (3.37) in $t \in J_{0}$ weighted with $\left|\widehat{g}_{w}(t)\right|$ : In view of (3.8) we have $\int_{J_{0}}\left|\widehat{g}_{w}(t)\right| \mathrm{d} t \ll \log \left(1+|b-a| q_{0}^{-1 / 2} r^{-1}\right)$ and thus we finally get, using the quantity $\|\widehat{\zeta}\|_{*, r}$ as defined in (2.6) for the weights $\zeta(x)$, the estimate

$$
I_{\Delta} \ll_{d} d_{Q} r^{d / 2} \log \left(1+|b-a| q_{0}^{-1 / 2} r^{-1}\right)\|\widehat{\zeta}\|_{*, r} .
$$

Applying (3.7) of Corollary 3.2 with (3.19), (3.30) and (3.38) we may now collect the results obtained so far as follows for the lattice point remainder of (3.5). We have

$$
\begin{aligned}
\mid \sum_{m \in \mathbb{Z}^{d}} & I_{[a, b]}(Q[m]) \mathrm{v}_{r}(m)-\int_{\mathbb{R}^{d}} I_{[a, b]}(Q[x]) \mathrm{v}_{r}(x) \mathrm{d} x \mid \\
& \ll_{d} I_{\theta}+d_{Q} r^{d / 2}\|\widehat{\zeta}\|_{*, r} \log \left(1+|b-a| q_{0}^{-1 / 2} r^{-1}\right)+w\|\mathrm{v}\|_{Q} r^{d-2} .
\end{aligned}
$$




\subsubsection{Estimation of $I_{\theta}$}

We shall now estimate the crucial error term $I_{\theta}$, see (3.22) and (3.23). At first we shall bound the theta series $\theta_{v}(t)$ uniformly in $v$ by another theta series in dimension $2 d$ in order to transform the problem to averages over functions on the space of lattices subject to an appropriate action of $\operatorname{SL}(2, \mathbb{R})$. We have

Lemma 3.3 Let $\theta_{v}(t)$ denote the theta function in (3.14) depending on $Q$, $r \in \mathbb{R}$ and $v \in \mathbb{R}^{d}$. For $r \geq 1, t \in \mathbb{R}$ the following bound holds uniformly in $v \in \mathbb{R}^{d}$

$$
\begin{aligned}
\left|\theta_{v}(t)\right| & \ll_{d}\left(\operatorname{det} Q_{+}\right)^{-1 / 4} r^{d / 2} \psi(r, t)^{1 / 2}, \text { where } \\
\psi(r, t) & \stackrel{\text { def }}{=} \sum_{m, n \in \mathbb{Z}^{d}} \exp \left\{-H_{t}(m, n)\right\}, \\
H_{t}(m, n) & \stackrel{\text { def }}{=} r^{2} Q_{+}^{-1}[m-4 t Q n]+r^{-2} Q_{+}[n],
\end{aligned}
$$

and $H_{t}(m, n)$ is a positive quadratic form on $\mathbb{Z}^{2 d}$. Note that $H_{t}(m, n)$ depends as well on the currently fixed dilating variable $r$ which we suppress here.

Proof For any $x, y \in \mathbb{R}^{d}$ the equalities

$$
\begin{aligned}
2\left(Q_{+}[x]+Q_{+}[y]\right) & =Q_{+}[x+y]+Q_{+}[x-y], \\
\langle Q(x+y), x-y\rangle & =Q[x]-Q[y]
\end{aligned}
$$

hold. Rearranging $\theta_{v}(z) \overline{\theta_{v}(z)}$ and using (3.43), we would like to use $m+n$ and $m-n$ as new summation variables on a lattice. But both vectors have the same parity, that is $m+n \equiv m-n \bmod 2$. Since they are dependent one has to consider the $2^{d}$ affine sublattices indexed by $\alpha=\left(\alpha_{1}, \ldots, \alpha_{d}\right)$ with $\alpha_{j} \in\{0,1\}$ for $1 \leq j \leq d$ :

$$
\mathbb{Z}_{\alpha}^{d} \stackrel{\text { def }}{=}\left\{m \in \mathbb{Z}^{d}: m \equiv \alpha \bmod 2\right\},
$$

where, for $m=\left(m_{1}, \ldots, m_{d}\right), m \equiv \alpha \bmod 2$ means $m_{j} \equiv \alpha_{j} \bmod 2$ for all $1 \leq j \leq d$. Thus writing

$$
\theta_{v, \alpha}(t) \stackrel{\text { def }}{=} \sum_{m \in \mathbb{Z}_{\alpha}^{d}} \exp \left[-\frac{1}{r^{2}} Q_{+}[m]-2 \pi \mathrm{i} t Q[m]+2 \pi \mathrm{i}\left\langle m, \frac{v}{r}\right\rangle\right],
$$

we obtain $\theta_{v}(t)=\sum_{\alpha} \theta_{v, \alpha}(t)$ and hence by the Cauchy-Schwarz inequality

$$
\left|\theta_{v}(t)\right|^{2} \leq 2^{d} \sum_{\alpha \in\{0,1\}^{d}}\left|\theta_{v, \alpha}(t)\right|^{2}
$$


Using (3.43) and the absolute convergence of $\theta_{v, \alpha}(t)$, we can write

$$
\begin{aligned}
\left|\theta_{v, \alpha}(t)\right|^{2} & =\sum_{m, n \in \mathbb{Z}_{\alpha}^{d}} \exp \left[-\frac{1}{r^{2}}\left(Q_{+}[m]+Q_{+}[n]\right)-2 \pi \mathrm{i} t(Q[m]-Q[n])-2 \pi \mathrm{i}\left\langle m-n, \frac{v}{r}\right\rangle\right] \\
& =\sum_{m, n \in \mathbb{Z}_{\alpha}^{d}} \exp \left[-\frac{2}{r^{2}}\left(Q_{+}[\bar{m}]+Q_{+}[\bar{n}]\right)-4 \pi \mathrm{i}\left\langle 2 t Q \bar{m}+\frac{v}{r}, \bar{n}\right\rangle\right]
\end{aligned}
$$

where $\bar{m}=\frac{m+n}{2}, \bar{n}=\frac{m-n}{2}$. Note that the map

$$
\bigcup_{\alpha \in\{0,1\}^{d}} \mathbb{Z}_{\alpha}^{d} \times \mathbb{Z}_{\alpha}^{d} \longrightarrow \mathbb{Z}^{d} \times \mathbb{Z}^{d}, \quad(m, n) \longmapsto\left(\frac{m+n}{2}, \frac{m-n}{2}\right)
$$

is a bijection. Therefore we get by (3.44)

$$
\begin{aligned}
\left|\theta_{v}(t)\right|^{2} & \ll_{d} \sum_{\alpha \in\{0,1\}^{d}} \sum_{m, n \in \mathbb{Z}_{\alpha}^{d}} \exp \left[-\frac{2}{r^{2}}\left(Q_{+}[\bar{m}]+Q_{+}[\bar{n}]\right)-4 \mathrm{i} \pi\left\langle 2 t Q \bar{m}+\frac{v}{r}, \bar{n}\right\rangle\right] \\
& =\sum_{\bar{m}, \bar{n} \in \mathbb{Z}^{d}} \exp \left[-\frac{2}{r^{2}}\left(Q_{+}[\bar{m}]+Q_{+}[\bar{n}]\right)-4 \mathrm{i} \pi\left\langle 2 t Q \bar{m}+\frac{v}{r}, \bar{n}\right\rangle\right] .
\end{aligned}
$$

In this double sum fix $\bar{n}$ and sum over $\bar{m} \in \mathbb{Z}^{d}$ first, and call the inner sum $\theta_{v}(t, \bar{n})$. Using (3.24) with $\Xi=2 \mathrm{i} Q_{+} r^{-2} / \pi$ and $v=-4 t Q \bar{n}+m$, we get for $\delta:=\left(\operatorname{det}\left(\frac{2}{\pi r^{2}} Q_{+}\right)\right)^{-1 / 2}$ by the symmetry of $Q$ and Poisson's formula (see [8], §46)

$$
\begin{aligned}
\theta_{v}(t, \bar{n}) & \stackrel{\text { def }}{=} \sum_{\bar{m} \in \mathbb{Z}^{d}} \exp \left[-\frac{2}{r^{2}}\left(Q_{+}[\bar{m}]+Q_{+}[\bar{n}]\right)-4 \pi \mathrm{i}\left\langle 2 t Q \bar{m}+\frac{v}{r}, \bar{n}\right\rangle\right] \\
& =\delta \sum_{m \in \mathbb{Z}^{d}} \exp \left[-\frac{\pi^{2} r^{2}}{2} Q_{+}^{-1}[m-4 t Q \bar{n}]-\frac{2}{r^{2}} Q_{+}[\bar{n}]-4 \pi \mathrm{i}\left\langle\frac{v}{r}, \bar{n}\right\rangle\right] .
\end{aligned}
$$

Thus, we have uniformly in $v \in \mathbb{R}^{d}$

$$
\left|\theta_{v}(t, \bar{n})\right| \leq \delta \sum_{m \in \mathbb{Z}^{d}} \exp \left\{-\frac{\pi^{2} r^{2}}{2} Q_{+}^{-1}[m-4 t Q \bar{n}]-\frac{2}{r^{2}} Q_{+}[\bar{n}]\right\} .
$$

Hence we obtain by (3.45) and (3.46)

$$
\left|\theta_{v}(t)\right|^{2} \ll_{d}\left(\operatorname{det} Q_{+}\right)^{-1 / 2} r^{d} \sum_{m, n \in \mathbb{Z}^{d}} \exp \left\{-G_{t}(m, n)\right\}
$$


where $G_{t}(m, n):=\frac{\pi^{2} r^{2}}{2} Q_{+}^{-1}[m-4 t Q n]+\frac{2}{r^{2}} Q_{+}[n]$. Since $\pi^{2} / 2>1$ we may bound $G_{t}(m, n)$ from below as follows:

$$
G_{t}(m, n) \geq r^{2} Q_{+}^{-1}[m-4 t Q n]+r^{-2} Q_{+}[n]=H_{t}(m, n)
$$

which proves the claimed estimate (3.40). Finally, observe that we can write

$$
H_{t}(m, n)=\left\|\left(\begin{array}{c}
r Q_{+}^{-\frac{1}{2}}(m-4 t Q n) \\
r^{-1} Q_{+} n
\end{array}\right)\right\|^{2},
$$

which shows that $H_{t}(m, n)$ is a positive definite quadratic form on $\mathbb{Z}^{2 d}$.

In view of Lemma 3.3 we can introduce the $2 d$-dimensional lattice

$$
\Lambda_{t} \stackrel{\text { def }}{=} D_{r Q} U_{4 t} Q \mathbb{Z}^{2 d},
$$

where

$$
D_{r Q}=\left(\begin{array}{ll}
r Q_{+}^{-\frac{1}{2}} & \\
& r^{-1} Q_{+}^{\frac{1}{2}}
\end{array}\right) \quad \text { and } \quad U_{4 t Q}=\left(\begin{array}{c}
\mathbb{1}_{d}-4 t Q \\
\mathbb{1}_{d}
\end{array}\right)
$$

in order to write $\psi(r, t)=\sum_{v \in \Lambda_{t}} \exp \left\{-\|v\|^{2}\right\}$ as the Siegel transform of $\exp \left\{-\|x\|^{2}\right\}$ evaluated at the lattice $\Lambda_{t}$. According to the Lipschitz principle in the Geometry of Numbers (see [50], Lemma 2, or [23], Lemma 3.1) one can show that $\psi(r, t) \ll_{d} \alpha\left(\Lambda_{t}\right)$, where $\alpha$ is the maximum over all $\alpha_{l^{-}}$ characteristics (see (2.2)). However, we choose to follow a more direct and transparent argument for the sake of clarity and motivate the relation between the $\alpha_{i}$-characteristics and the successive minima of a lattice for the convenience of the reader. The following Lemma 3.4 (with $\varepsilon=1$ ) reduces the problem of estimating the theta series (3.41) to the problem of counting lattice points as follows

$$
\psi(r, t) \asymp_{d} \#\left\{w \in \Lambda_{t}:\|w\|_{\infty} \leq 1\right\} \ll_{d} \#\left\{w \in \Lambda_{t}:\|w\| \leq d^{1 / 2}\right\} .
$$

Lemma 3.4 Let $\Lambda$ be a lattice in $\mathbb{R}^{d}$. Assume that $0<\varepsilon \leq 1$, then

$$
\exp \{-d \varepsilon\} \# \mathcal{H} \leq \sum_{v \in \Lambda} \exp \left\{-\varepsilon\|v\|^{2}\right\} \ll_{d} \varepsilon^{-d / 2} \# \mathcal{H},
$$

where $\mathcal{H}:=\left\{v \in \Lambda:\|v\|_{\infty}<1\right\}$. 
Proof The lower bound for the sum is obvious by restricting summation to the set of elements in $\mathcal{H}$. As for the upper bound introduce for $\mu=\left(\mu_{1}, \ldots, \mu_{d}\right) \in$ $\mathbb{Z}^{d}$ the sets

$$
B_{\mu} \stackrel{\text { def }}{=}\left[\mu_{1}-\frac{1}{2}, \mu_{1}+\frac{1}{2}\right) \times \cdots \times\left[\mu_{d}-\frac{1}{2}, \mu_{d}+\frac{1}{2}\right)
$$

such that $\mathbb{R}^{d}=\bigcup_{\mu \in \mathbb{Z}^{d}} B_{\mu}$. For any fixed $w^{*} \in \mathcal{H}_{\mu}:=\Lambda \cap B_{\mu}$ we have $w-w^{*} \in \mathcal{H}$ for all $w \in \mathcal{H}_{\mu}$. Hence we conclude for any $\mu \in \mathbb{Z}^{d}$

$$
\# \mathcal{H}_{\mu} \leq \# \mathcal{H} \text {. }
$$

Since $x \in B_{\mu}$ implies $\|x\|_{\infty} \geq\|\mu\|_{\infty} / 2$, we obtain

$$
\sum_{v \in \Lambda} \mathrm{e}^{-\varepsilon\|v\|^{2}} \leq \sum_{v \in \Lambda} \mathrm{e}^{-\varepsilon\|v\|_{\infty}^{2}} \leq \sum_{\mu \in \mathbb{Z}^{d}} \sum_{v \in \Lambda \cap B_{\mu}} \mathrm{e}^{-\frac{\varepsilon}{4}\|\mu\|_{\infty}^{2}} \leq \# \mathcal{H} \sum_{\mu \in \mathbb{Z}^{d}} \mathrm{e}^{-\frac{\varepsilon}{4}\|\mu\|^{2}} \ll d \varepsilon^{-d / 2} \# \mathcal{H} .
$$

This concludes the proof of Lemma 3.4.

\section{Functions on the space of lattices and geometry of numbers}

Let $n \in \mathbb{N}^{+}$be fixed (later to be chosen as $n=2 d$ ) and for every integer $l$ with $1 \leq l \leq n$ we fix a quasinorm $|\cdot|_{l}$ on the exterior product $\wedge^{l} \mathbb{R}^{n}$. Let $L$ be a subspace of $\mathbb{R}^{n}$ and $\Delta$ a lattice in $L$ (i.e. $\Delta$ is a free $\mathbb{Z}$-module of full rank $\operatorname{dim} L$ ), then any two bases of $\Delta$ are related by a unimodular transformation, that is, if $u_{1}, \ldots, u_{l}$ and $v_{1}, \ldots, v_{l}$ are two bases of $\Delta$, where $l=\operatorname{dim} L$, then $v_{1} \wedge \cdots \wedge v_{l}= \pm u_{1} \wedge \cdots \wedge u_{l}$, which implies that the expression $\left|v_{1} \wedge \cdots \wedge v_{l}\right|_{l}$ is independent of the choice of basis.

Let $\Delta$ be a lattice in $\mathbb{R}^{n}$, we say that a subspace $L$ of $\mathbb{R}^{n}$ is $\Delta$-rational if $L \cap \Delta$ is a lattice in $L$. For any $\Delta$-rational subspace $L$, we denote by $d_{\Delta}(L)$, or simply by $d(L)$, the quasinorm $\left|u_{1} \wedge \ldots \wedge u_{l}\right|_{l}$ where $\left\{u_{1}, \ldots, u_{l}\right\}, l=\operatorname{dim} L$, is a basis of $L \cap \Delta$ over $\mathbb{Z}$. For $L=\{0\}$ we write $d(L):=1$. If the quasinorms $|\cdot|_{l}$ are the norms on $\wedge^{l} \mathbb{R}^{n}$ induced from the standard Euclidean norm on $\mathbb{R}^{n}$, then $d(L)$ is equal to the determinant (or discriminant) $\operatorname{det}(L \cap \Delta)$ of the lattice $L \cap \Delta$, that is the volume of $L /(L \cap \Delta)$. In particular, in this case the lattice $\Delta$ is said to be unimodular if and only if $d_{\Delta}\left(\mathbb{R}^{n}\right)=1$. Also in this case $d(L) d(M) \geq d(L \cap M) d(L+M)$ for any two $\Delta$-rational subspaces $L$ and $M$ (see Lemma 5.6 in [23]), but any two quasinorms on $\wedge^{l} \mathbb{R}^{n}$ are equivalent, which proves

Lemma 4.1 There is a constant $C \geq 1$ depending only on the quasinorm $|\cdot|_{l}$ and not on $\Delta$ such that

$$
C^{2} d(L) d(M) \geq d(L \cap M) d(L+M)
$$


for any two $\Delta$-rational subspaces $L$ and $M$.

Let us introduce the following notations for $0 \leq l \leq n$,

$$
\begin{aligned}
\alpha_{l}(\Delta) & \stackrel{\text { def }}{=} \sup \left\{d(L)^{-1}: L \text { is a } \Delta \text {-rational subspace of dimension } l\right\}, \\
\alpha(\Delta) & \stackrel{\text { def }}{=} \max _{0 \leq l \leq n} \alpha_{l}(\Delta) .
\end{aligned}
$$

This extends the earlier definition (2.2) of $\alpha_{l}(\Delta)$ in the introduction of Sect. 2 to the case of general seminorms on $\wedge^{l} \mathbb{R}^{n}$. In this section the functions $\alpha_{l}$ and $\alpha$ will be based on standard Euclidean norms, that is, we have $d(L)=\operatorname{det}(L \cap \Delta)$.

In the following we shall use some facts from the Geometry of Numbers and the classical reduction theory for lattices in $\mathbb{R}^{n}$, see Davenport [17], Siegel [52], Cassels [14] and Einsiedler-Ward [24]. The successive minima of a lattice $\Lambda$ are the numbers $M_{1}(\Lambda) \leq \cdots \leq M_{n}(\Lambda)$ defined as follows: $M_{j}(\Lambda)$ is the infimum of $\lambda>0$ such that the set $\{v \in \Lambda:\|v\|<\lambda\}$ contains $j$ linearly independent vectors and in particular $M_{1}(\Lambda)$ is the shortest non-zero vector of the lattice $\Lambda$. It is easy to see that these infima are attained, that is, there exist linearly independent vectors $v_{1}, \ldots, v_{n} \in \Lambda$ such that $\left\|v_{j}\right\|=M_{j}(\Lambda)$ for all $j=1, \ldots, n$. Moreover, as a consequence of the reduction algorithm of Korkine and Zolotareff (see [35-37]) the $\alpha_{l}$-characteristic and the successive minima are related according to $\alpha_{l}(\Lambda) \asymp_{d}\left(M_{1}(\Lambda) \ldots M_{l}(\Lambda)\right)^{-1}$ (see [24], Chapter 1, Theorem 15).

Lemma 4.2 Let $F$ be a norm in $\mathbb{R}^{n}$ and denote by $M_{1} \leq \cdots \leq M_{n}$ the successive minima with respect to $F$. Let $\Lambda$ be a lattice in $\mathbb{R}^{n}$, then

$$
\alpha_{l}(\Lambda) \asymp_{n}\left(M_{1}(\Lambda) \cdots M_{l}(\Lambda)\right)^{-1}, \quad l=1, \ldots, n .
$$

Moreover, for any $\mu>0$, if $1 \leq j \leq n$ is such that $M_{j}(\Lambda) \leq \mu<M_{j+1}(\Lambda)$, where the right-hand side is omitted if $j=n$, then

$$
\#\{v \in \Lambda: F(v) \leq \mu\} \asymp_{n} \mu^{j} \alpha_{j}(\Lambda) .
$$

Proof First we prove the lower bound. We may assume that $M_{j}(\Lambda) \leq \mu<$ $M_{j+1}(\Lambda)$, the right-hand side being omitted if $j=n$. Let $v_{1}, \ldots, v_{n}$ denote the elements in $\Lambda$ corresponding to the successive minima $M_{i}(\Lambda), i=1, \ldots, n$. For $m_{1}, \ldots, m_{j} \in \mathbb{Z}$ with $\left|m_{i}\right| \leq j^{-1} \mu F\left(v_{i}\right)^{-1}$ notice that $v=m_{1} v_{1}+\ldots+$ $m_{j} v_{j}$ satisfies $F(v) \leq \mu$, thus

$$
N(\mu) \stackrel{\text { def }}{=} \#\{v \in \Lambda: F(v) \leq \mu\} \gg_{m} \mu^{j}\left(M_{1}(\Lambda) \cdots M_{j}(\Lambda)\right)^{-1} .
$$

The upper bound is also proven in Davenport [17] (see Lemma 1). We include the short argument here for the sake of completeness: Let $w_{1}, \ldots, w_{n}$ be an 
integral basis of $\Lambda$ such that $v_{i}$ is linearly dependent on $w_{1}, \ldots, w_{i}$ for any $i=1, \ldots, n$. Consequently any lattice point $v \in \Lambda$ with $F(v)<M_{j+1}$ is linearly dependent on $w_{1}, \ldots, w_{j}$ and hence any element $v \in \Lambda$ with $F(v) \leq \mu$ can be written as $v=m_{1} w_{1}+\ldots+m_{j} w_{j}$ with $m_{i} \in \mathbb{Z}$. Suppose $v^{\prime} \in \Lambda$ is another element with $F\left(v^{\prime}\right) \leq \mu$ and write $v^{\prime}=m_{1}^{\prime} w_{1}+\ldots m_{j}^{\prime} w_{j}$ with $m_{i}^{\prime} \in \mathbb{Z}$. Now define positive integers $v_{1}, \ldots, v_{j}$ by

$$
2^{v_{i}-1} \leq \frac{2 \mu}{M_{i}(\Lambda)}<2^{v_{i}}
$$

and observe that $v_{1} \geq v_{2} \geq \ldots \geq v_{j}$. Assuming for the moment that $m_{i} \equiv m_{i}^{\prime}$ $\bmod 2^{v_{i}}$ for every $i=1, \ldots, j$ and let $i_{0}$ denote the largest index $i_{0}$ such that $m_{i_{0}} \neq m_{i_{0}}^{\prime}$. Then $x:=2^{-v_{i_{0}}}\left(v-v^{\prime}\right)$ is an element of $\Lambda$ and linearly independent of $w_{1}, \ldots, w_{i_{0}-1}$. This implies $F(x) \geq M_{i_{0}}(\Lambda)$. On the other hand we have

$$
F(x)=2^{-v_{i}} F\left(v-v^{\prime}\right) \leq 2^{-v_{i}}\left(F(v)+F\left(v^{\prime}\right)\right) \leq 2^{-v_{i}} 2 \mu<M_{i_{0}}(\Lambda)
$$

by (4.7). This contradiction shows that there is at most one lattice point in $\Delta$, implying that the coordinates $m_{1}, \ldots, m_{j}$ lie in the same residue classes modulo $2^{v_{1}}, 2^{v_{2}}, \ldots, 2^{v_{j}}$ respectively. Hence, the number of lattice points $N(\mu)$ in (4.6) is bounded from above by the number of all residue classes, i.e. by $2^{v_{1}} 2^{v_{2}} \ldots 2^{v_{j}} \leq(4 \mu)^{j}\left(M_{1}(\Lambda) \ldots M_{j}(\Lambda)\right)^{-1}$. This shows the upper bound in (4.5).

Lemma 4.3 (Davenport [17]) Let $\Lambda=g \mathbb{Z}^{n}$ and $\Lambda^{\prime}=\left(g^{-1}\right)^{T} \mathbb{Z}^{n}$ denote dual lattices of rank $n$, then for all $j=1, \ldots, n$ we have

$$
1 \leq M_{j}(\Lambda) M_{n+1-j}\left(\Lambda^{\prime}\right) \ll_{n} 1 .
$$

This is a variant of Lemma 2 of Davenport [17] for the Euclidean norm. Again, for the reader's convenience, we include the short argument here.

Proof. Let $v_{1}, \ldots, v_{n} \in \Lambda$, resp. $v_{1}^{\prime}, \ldots, v_{n}^{\prime} \in \Lambda^{\prime}$, be linearly independent such that $\left\|v_{i}\right\|=M_{i}(\Lambda)$, resp. $\left\|v_{i}^{\prime}\right\|=M_{i}\left(\Lambda^{\prime}\right)$. Then $v_{1}, \ldots, v_{j}$ cannot be orthogonal to all lattice points $v_{1}^{\prime}, \ldots, v_{n+1-j}^{\prime}$, otherwise they would fail to be independent. Thus, we have $\left\langle v_{i}, v_{k}^{\prime}\right\rangle \neq 0$ for some $i=1, \ldots, j$ and $k=$ $1, \ldots, n+1-j$, which implies that

$$
M_{j}(\Lambda) M_{n+1-j}\left(\Lambda^{\prime}\right) \geq M_{i}(\Lambda) M_{k}\left(\Lambda^{\prime}\right)=\left\|v_{i}\right\|\left\|v_{k}^{\prime}\right\| \geq\left|\left\langle v_{i}, v_{k}^{\prime}\right\rangle\right| \geq 1
$$

because of duality. The right-hand side of (4.8) follows from (4.4) with $l=n$, which is known as Minkowski's inequality. Indeed, $\operatorname{det}(\Lambda)=\alpha_{n}(\Lambda)^{-1} \asymp_{n}$ 
$M_{1}(\Lambda) \ldots M_{n}(\Lambda)$ and since $\operatorname{det}(\Lambda) \operatorname{det}\left(\Lambda^{\prime}\right)=1$ we conclude that

$$
M_{j}(\Lambda) M_{n+1-j}\left(\Lambda^{\prime}\right) \ll_{n} \prod_{h=1, h \neq j}^{n}\left(M_{h}(\Lambda) M_{n+1-h}\left(\Lambda^{\prime}\right)\right)^{-1} \ll_{n} 1 .
$$

\subsection{Sympletic structure of $\Lambda_{t}$}

In the following we shall apply the previous results from the Geometry of Numbers to the special $2 d$-dimensional lattice $\Lambda_{t}$ introduced in (3.47). The symplectic structure of $\Lambda_{t}$ will allow us to establish a majorizing relation between the theta series (3.41) and the $\alpha_{d}$-characteristic of $\Lambda_{t}$, see (4.14). To do this, we shall apply Lemma 4.2 combined with Lemma 4.3 as follows. (We note that the results of this section remain valid regardless of whether $r \geq q^{1 / 2}$ or not.)

Lemma 4.4 Let $\Lambda_{t}$ be the lattice defined in (3.47). Then we have for any $t \in \mathbb{R}$

$$
\begin{aligned}
M_{j}\left(\Lambda_{t}\right) M_{2 d+1-j}\left(\Lambda_{t}\right) \asymp_{d} 1 \quad(j=1, \ldots, d), \\
M_{1}\left(\Lambda_{t}\right) \leq \cdots \leq M_{d}\left(\Lambda_{t}\right) \ll_{d} 1 \leq M_{d+1}\left(\Lambda_{t}\right) \leq \cdots \leq M_{2 d}\left(\Lambda_{t}\right),
\end{aligned}
$$

and the lower bound

$$
M_{1}\left(\Lambda_{t}\right) \geq \min \left\{r^{-1} q_{0}^{1 / 2}, r q^{-1 / 2}\right\} .
$$

Corollary 4.5 As a consequence, we find for $\mu \geq 1$

$$
\begin{aligned}
& \#\left\{v \in \Lambda_{t}:\|v\| \leq \mu\right\} \ll_{d} \mu^{2 d} \alpha_{d}\left(\Lambda_{t}\right), \\
& \alpha\left(\Lambda_{t}\right)=\max \left\{\alpha_{j}\left(\Lambda_{t}\right): j=1, \ldots, 2 d\right\} \asymp_{d} \alpha_{d}\left(\Lambda_{t}\right)
\end{aligned}
$$

and

$$
\psi(r, t) \ll_{d} \alpha_{d}\left(\Lambda_{t}\right)
$$

Proof of Lemma 4.4 First we prove (4.9). Let

$$
J \stackrel{\text { def }}{=}\left(\begin{array}{l}
\mathbb{1}_{d} \\
-\mathbb{1}_{d}
\end{array}\right)
$$

and consider the lattice

$$
\Lambda_{t}^{\prime}=J D_{r Q} U_{4 t} J^{-1} \mathbb{Z}^{2 d}
$$

Then $J D_{r Q} U_{4 t} J^{-1}=D_{r Q}^{-1} U_{-4 t}^{T}$ and hence $\Lambda_{t}^{\prime}$ is the lattice dual to $\Lambda_{t}$ in the sense of Lemma 4.3. We claim that they have identical successive minima. 
To this end, note that for any $N=(m, \bar{m})^{T} \in \mathbb{Z}^{2 d}$

$$
\left\|D_{r Q} U_{4 t} Q N\right\|=\left\|J^{-1} J D_{r Q} U_{4 t} J^{-1} J N\right\|=\left\|D_{r Q}^{-1} U_{-4 t}^{T} J N\right\|,
$$

where we use that $J$ is an orthogonal matrix. Since $J \mathbb{Z}^{2 d}=\mathbb{Z}^{2 d}$, the Eq. (4.15) implies that the successive minima of $\Lambda_{t}$ and $\Lambda_{t}^{\prime}$ are identical and by Lemma 4.3 we conclude $M_{j}\left(\Lambda_{t}\right) M_{2 d+1-j}\left(\Lambda_{t}\right) \asymp_{d} 1$ for $j=1, \ldots, d$.

To prove (4.10) we note that $M_{d} \leq M_{d+1}$ and $1 \leq M_{d}\left(\Lambda_{t}\right) M_{d+1}\left(\Lambda_{t}\right) \ll_{d} 1$ implies

$$
M_{j}\left(\Lambda_{t}\right) \leq M_{d}\left(\Lambda_{t}\right) \ll_{d} 1 \text { and } 1 \leq M_{d+1}\left(\Lambda_{t}\right) \leq M_{d+j}\left(\Lambda_{t}\right)
$$

for all $j=1, \ldots, d$. Thus, it remains to show the lower bound (4.11) for $M_{1}\left(\Lambda_{t}\right)$ : Take $m, \bar{m} \in \mathbb{Z}^{d}$ with $M_{1}\left(\Lambda_{t}\right)=\left\|D_{r Q} U_{4 t}(m, \bar{m})\right\|=$ $H_{t}(m, \bar{m})^{1 / 2}$, where $H_{t}$ denotes the special norm (3.42) in the theta series (3.41). If $\bar{m} \neq 0$, then we have $M_{1}\left(\Lambda_{t}\right) \geq r^{-1}\left\|Q_{+}^{1 / 2} \bar{m}\right\| \geq q_{0}^{1 / 2} r^{-1}$, but otherwise $M_{1}\left(\Lambda_{t}\right)=r\left\|Q_{+}^{-1 / 2} m\right\| \geq r q^{-1 / 2}$.

Proof of Corollary 4.5 We begin with proving (4.12) as follows. Recall that $\mu \geq 1$ and let $2 d \geq j \geq 1$ denote the maximal integer with $M_{j}\left(\Lambda_{t}\right) \leq \mu$. Then Lemma 4.2 implies

$$
\#\left\{v \in \Lambda_{t}:\|v\| \leq \mu\right\} \ll_{d} \mu^{j} \alpha_{j}\left(\Lambda_{t}\right) \leq \mu^{2 d} \alpha_{d}\left(\Lambda_{t}\right),
$$

since we have $M_{j}\left(\Lambda_{t}\right) \geq \ldots \geq M_{d+1}\left(\Lambda_{t}\right) \gg 1$ if $j>d$ and $\mu<$ $M_{j+1}\left(\Lambda_{t}\right) \leq \ldots \leq M_{d}\left(\Lambda_{t}\right) \ll_{d} 1$ if $j<d$. In the case $\mu<M_{1}\left(\Lambda_{t}\right)$ the inequality in (4.12) holds trivially. Moreover, this argument also proves (4.13). Finally, the estimate (4.14) follows from the relation (3.49) combined with (4.12) for $\mu=d^{1 / 2}$.

For arbitrary $t \in \mathbb{R}$ the following bounds hold independently of the Diophantine properties of $Q$.

Lemma 4.6 Denote by $\Delta$ the lattice $Q_{+}^{1 / 2} \mathbb{Z}^{d}$, then

$$
\sup _{t \in \mathbb{R}} \alpha_{d}\left(D_{s} U_{4 t} Q \mathbb{Z}^{2 d}\right) \ll_{d} \varphi_{Q}(s),
$$

where $D_{S} Q$ and $U_{4 t} Q$ are defined as in (3.48) and

$$
\varphi_{Q}(s) \stackrel{\text { def }}{=} s^{d}|\operatorname{det} Q|^{-1 / 2} \prod_{j: M_{j}(\Delta)>s}\left(s^{-2} M_{j}(\Delta)^{2}\right), s>0 .
$$

In particular, it follows that

$$
\varphi_{Q}(s) \ll_{d} s^{d}|\operatorname{det} Q|^{-1 / 2}, \text { if }|s| \geq q^{1 / 2},
$$


and for small $t$ we get

$$
\begin{array}{ll}
\alpha_{d}\left(D_{s Q} U_{4 t} Q \mathbb{Z}^{2 d}\right) \ll_{d}|\operatorname{det} Q|^{1 / 2}\left(s^{-1}+|t s|\right)^{d}, & \text { if } q_{0}^{1 / 2}|t s| \geq 1, \\
\alpha_{d}\left(D_{s Q} U_{4 t} Q \mathbb{Z}^{2 d}\right) \ll_{d}|\operatorname{det} Q|^{-1 / 2} \max \left\{1,(\sqrt{q} / s)^{d}\right\}|t s|^{-d}, & \text { if } q^{1 / 2}|t s| \leq 1
\end{array}
$$

We emphasize that these estimates will be used for a wide range of $s>0$ (depending on the blow-up parameter $r \geq q^{1 / 2}$ ), see e.g. the proof of Lemma 6.2, and for small $t$ as well (by which we mean $r^{-1} q_{0}^{-1 / 2}<t<T_{-}$ as stated in Theorem 2.2).

Proof In this proof we replace the definition of $\Lambda_{t}$, see (3.47), by $\Lambda_{t}=$ $D_{s Q} U_{4 t} Q \mathbb{Z}^{2 d}$, i.e. $r$ has to be replaced by $s$. If $1 / 8<M_{1}\left(\Lambda_{t}\right)$, then we have

$$
\alpha_{d}\left(\Lambda_{t}\right) \asymp_{d}\left(M_{1}\left(\Lambda_{t}\right) \ldots M_{d}\left(\Lambda_{t}\right)\right)^{-1} \ll_{d} \#\left\{v \in \Lambda_{t}:\|v\| \leq 1 / 8\right\} .
$$

Otherwise, there exists an integer $j=1, \ldots, d$ with $M_{j}\left(\Lambda_{t}\right) \leq 1 / 8<$ $M_{j+1}\left(\Lambda_{t}\right)$, since $1 \leq M_{d+1}\left(\Lambda_{t}\right)$ holds by (4.10). Now, taking $\mu=1 / 8$ in (4.5) of Lemma 4.2 shows that

$$
\begin{aligned}
\alpha_{d}\left(\Lambda_{t}\right) & \asymp_{d}\left(M_{1}\left(\Lambda_{t}\right) \ldots M_{d}\left(\Lambda_{t}\right)\right)^{-1} \\
& \ll\left(M_{1}\left(\Lambda_{t}\right) \ldots M_{j}\left(\Lambda_{t}\right)\right)^{-1} \asymp_{d} \#\left\{v \in \Lambda_{t}:\|v\| \leq 1 / 8\right\},
\end{aligned}
$$

i.e. (4.21) holds also in the second case. Recalling again (3.42), we see that the right-hand side of (4.21) is the same as the number all lattice points $m, \bar{m} \in \mathbb{Z}^{d}$ satisfying

$$
H_{t}[m, \bar{m}]=s^{2} Q_{+}^{-1}[m-4 t Q \bar{m}]+s^{-2} Q_{+}[\bar{m}] \leq 1 / 64,
$$

where the positive form $H_{t}[\cdot, \cdot]$ is defined as in (3.42), but here again $r$ has to be replaced by $s$.

Proof of (4.16) If (4.22) holds, then $\left\|Q_{+}^{1 / 2} \bar{m}\right\| \leq s / 2$, which has again by Lemma 4.2 at most $\ll_{d} \prod_{j: M_{j}(\Delta) \leq s}\left(s M_{j}(\Delta)^{-1}\right)$ integral solutions. Similarly, for fixed $\bar{m}$ the triangle inequality combined with (4.22) implies

$$
\left\|s Q_{+}^{-1 / 2}\left(m_{1}-m_{2}\right)\right\| \leq \sqrt{H_{t}\left[m_{1}, \bar{m}\right]}+\sqrt{H_{t}\left[m_{2}, \bar{m}\right]} \leq 1 .
$$

Thus, for fixed $\bar{m}$, the number of pairs $(m, \bar{m})$ for which (4.22) holds is bounded by the number of elements $v$ in the dual lattice $\Delta^{\prime}=Q_{+}^{-1 / 2} \mathbb{Z}^{d}$ to $\Delta$ such that $\|v\| \leq s^{-1}$. Since the successive minima for this dual lattice are determined 
by Lemma 4.3, we may use Lemma 4.2, inequality (4.5), again to determine the upper bound

$$
\ll_{d} \prod_{j: M_{j}\left(\Delta^{\prime}\right) \leq s^{-1}}\left(s M_{j}\left(\Delta^{\prime}\right)\right)^{-1} \leq \prod_{j: M_{j}(\Delta) \geq s}\left(s^{-1} M_{j}(\Delta)\right)
$$

for this number as well. The product of both numbers yields the bound $\alpha_{d}\left(\Lambda_{t}\right) \ll_{d} \#\left\{v \in \Lambda_{t}:\|v\| \leq 1 / 2\right\} \ll_{d} s^{d}\left(\prod_{j=1}^{d} M_{j}(\Delta)\right)^{-1}\left(\prod_{j: M_{j}(\Delta) \geq s}\left(s^{-2} M_{j}(\Delta)^{2}\right)\right)$.

Finally, using Lemma 4.2 in form of $\left(\prod_{j=1}^{d} M_{j}(\Delta)\right)^{-1} \asymp_{d} \alpha_{d}(\Delta)=$ $\mid$ det $\left.Q\right|^{1 / 2}$ shows the claimed bound in (4.16). Also the inequality (4.18) follows immediately from (4.17).

Proof of (4.19) Assume $q_{0}^{1 / 2}|t s| \geq 1$ and $q_{0} \geq 1$. If $m=0$ we conclude that $\|\bar{m}\| \leq|4 t s|\left\|Q_{+}^{1 / 2} \bar{m}\right\| \leq 1 / 8$. Hence $\bar{m}=0$. For any fixed $m \neq 0$ the triangle inequality implies that there is at most one element $\bar{m} \in \mathbb{Z}^{d}$ with (4.22). Furthermore, we get $\left(\left\|Q_{+}^{-1 / 2} m\right\|-1 /(8 s)\right) \leq\left\|4 t Q_{+}^{1 / 2} \bar{m}\right\|$ for that pair $(m, \bar{m})$. This implies

$$
1 / 8 \geq \sqrt{H_{t}(m, \bar{m})} \geq s^{-1}\left\|Q_{+}^{1 / 2} \bar{m}\right\| \geq\left(\left\|Q_{+}^{-1 / 2} m\right\|-1 /(8 s)\right) /|4 t s|
$$

and hence $\left\|Q_{+}^{-1 / 2} m\right\| \leq\left(s^{-1}+|4 t s|\right) / 8$. Thus

$$
\#\left\{v \in \Lambda_{t}:\|v\|^{2} \leq 1 / 4\right\} \ll_{d}\left(s^{-1}+|t s|\right)^{d}|\operatorname{det} Q|^{1 / 2} .
$$

Proof of (4.20) As in the previous case, (4.22) implies by the triangle inequality that

$$
\left|\left\|Q_{+}^{-1 / 2} m\right\|-\left\|4 t Q_{+}^{1 / 2} S \bar{m}\right\|\right| \leq(8 s)^{-1}
$$

and together with $q^{1 / 2}|t s| \leq 1$ also $|4 t s| s^{-1}\left\|Q_{+}^{1 / 2} \bar{m}\right\| \leq|4 t s| / 8 \leq(2 q)^{-1 / 2}$. Moreover one of these inequalities is strict and therefore we have

$$
q^{-1 / 2}\|m\| \leq\left\|Q_{+}^{-1 / 2} m\right\|<(2 s)^{-1}+\left(2 q^{1 / 2}\right)^{-1} .
$$

If $s \geq q^{1 / 2}$, this leads to a contradiction unless $m=0$. Hence, the possible solutions for $\bar{m}$ in (4.23) satisfy $\left\|Q_{+}^{1 / 2} \bar{m}\right\| \leq|32 t s|^{-1}$ which, as in the proof of (4.16), has at most $\ll_{d}|\operatorname{det} Q|^{-1 / 2}|t s|^{-\bar{d}}$ solutions. In the second case, i.e. if $s<q^{1 / 2}$, the inequality (4.24) has at most $\ll_{d}\left(q^{1 / 2} / s\right)^{d}$ solutions for $m$. Now any possible $\bar{m}$ must satisfy

$$
\left\|Q_{+}^{1 / 2} \bar{m}\right\| \leq|32 t s|^{-1}+|4 t|^{-1}\left\|Q_{+}^{-1 / 2} m\right\| \leq|2 t s|^{-1}
$$


again, which completes the proof of (4.20) in view of (4.21).

\subsection{Approximation by compact subgroups}

In Sect. 5 we shall develop mean-value estimates for fractional moments of the $\alpha_{d}$-characteristic of the lattice $\Lambda_{t}$ introduced in (3.47). In order to apply techniques from harmonic analysis, we will rewrite the family $\left\{\Lambda_{t}\right\}_{t \in \mathbb{R}}$ as an orbit of a single lattice by means of elements of the one-parameter subgroups $\mathrm{D}:=\left\{d_{r}: r>0\right\}$ and $\mathrm{U}:=\left\{u_{t}: t \in \mathbb{R}\right\}$ of $\mathrm{SL}(2, \mathbb{R})$, where

$$
d_{r} \stackrel{\text { def }}{=}\left(\begin{array}{cc}
r & 0 \\
0 & r^{-1}
\end{array}\right), \quad u_{t} \stackrel{\text { def }}{=}\left(\begin{array}{cc}
1 & -t \\
0 & 1
\end{array}\right)
$$

and then approximate the subgroup $\mathrm{U}$ locally by the compact subgroup $\mathrm{K}=$ $\mathrm{SO}(2)=\left\{k_{\theta}: \theta \in[0,2 \pi]\right\}$ parameterized, as usual, by elements

$$
k_{\theta} \stackrel{\text { def }}{=}\left(\begin{array}{cc}
\cos \theta & -\sin \theta \\
\sin \theta & \cos \theta
\end{array}\right) .
$$

Let $S$ be an orthogonal matrix such that $S Q Q_{+}^{-1} S^{T}=Q_{0}$, where $Q_{0}$ denotes the signature matrix corresponding to $Q$, that is $Q_{0}=$ $\operatorname{diag}(1, \ldots, 1,-1, \ldots,-1)$. A short computation shows that

$$
D_{r Q} U_{4 t} Q=\left(\begin{array}{ll}
S^{T} & \\
& S^{T}
\end{array}\right) d_{r} u_{4 t}\left(\begin{array}{ll}
S Q_{+}^{-1 / 2} & \\
& S Q_{+}^{1 / 2}
\end{array}\right),
$$

where we embed $\operatorname{SL}(2, \mathbb{R})$ into $\operatorname{SL}(2 d, \mathbb{R})$ according to the following action

$$
\left(\begin{array}{ll}
a & b \\
c & d
\end{array}\right) \longmapsto\left(\begin{array}{cccc}
a & \mathbb{1}_{d} & b & Q_{0} \\
c & Q_{0} & d \mathbb{1}_{d}
\end{array}\right)
$$

Define the $2 d$-dimensional lattice

$$
\Lambda_{Q} \stackrel{\text { def }}{=}\left(\begin{array}{cc}
S Q_{+}^{-1 / 2} & \\
& S Q_{+}^{1 / 2}
\end{array}\right) \mathbb{Z}^{2 d},
$$

then as claimed,

$$
\Lambda_{t}=\left(\begin{array}{cc}
S^{T} & \\
& S^{T}
\end{array}\right) d_{r} u_{4 t} \Lambda_{Q}
$$


Moreover, since $S$ is orthogonal and $\alpha_{i}$ is invariant under left multiplication by orthogonal matrices we observe for any $i=1, \ldots, 2 d$ that

$$
\alpha_{i}\left(\Lambda_{t}\right)=\alpha_{i}\left(d_{r} u_{4 t} \Lambda_{Q}\right)
$$

Lemma 4.7 With respect to the embedding of $\operatorname{SL}(2, \mathbb{R})$ defined in (4.27) we have for $t \in \mathbb{R}, s \geq 1$ and any $2 d$-dimensional lattice $\Lambda$ in $\mathbb{R}^{2 d}$

$$
\alpha_{j}\left(d_{s} u_{t} \Lambda\right) \ll_{d}\left(1+t^{2}\right)^{\frac{j}{2}} \alpha_{j}\left(d_{s} k_{\theta} \Lambda\right), \quad j=1, \ldots, 2 d,
$$

where $\theta=\arctan t$.

Proof Suppose the signature of $Q$ is $(p, q)$ and let $(v, w) \in \mathbb{R}^{d} \times \mathbb{R}^{d}$, thought of as a column vector with coordinates $v_{1}, \ldots, v_{d}, w_{1}, \ldots, w_{d}$, then

$$
\left\|d_{s} u_{t}(v, w)\right\|^{2}=\sum_{i=1}^{p}\left\|d_{s} u_{t}\left(v_{i}, w_{i}\right)\right\|^{2}+\sum_{i=p+1}^{d}\left\|d_{s} u_{-t}\left(v_{i}, w_{i}\right)\right\|^{2}
$$

Let $x, y \in \mathbb{R}$. Note that $y+t x=\left(1+t^{2}\right) y+t(x-t y)$, which implies that

$$
(y+t x)^{2} \leq 2\left(1+t^{2}\right)^{2}(y)^{2}+2 t^{2}(x-t y)^{2},
$$

and therefore we find

$$
s^{2}(x-t y)^{2}+s^{-2}(y+t x)^{2} \leq 2\left(1+t^{2}\right)^{2}\left(s^{2}(x-t y)^{2}+s^{-2} y^{2}\right),
$$

provided that $s \geq 1$. Taking $\theta=\arctan t$ and noting that $\cos (\theta)=\left(t^{2}+1\right)^{-1 / 2}$, resp. $\sin (\theta)=t\left(t^{2}+1\right)^{-1 / 2}$, we see that (4.33) can be written as

$$
\left\|d_{s} k_{\theta}(x, y)\right\|^{2} \leq 2\left(1+t^{2}\right)\left\|d_{s} u_{t}(x, y)\right\|^{2},
$$

and it is easy to see, along the same lines as before, that

$$
\left\|d_{s} k_{\theta}^{T}(x, y)\right\|^{2} \leq 2\left(1+t^{2}\right)\left\|d_{s} u_{-t}(x, y)\right\|^{2} .
$$

Hence, we obtain in view of (4.32) that

$$
\left\|d_{s} k_{\theta}(v, w)\right\|^{2} \leq 2\left(1+t^{2}\right)\left\|d_{s} u_{t}(v, w)\right\|^{2},
$$

from which we deduce that $\left(1+t^{2}\right)^{i / 2} M_{i}\left(d_{s} u_{t} \Lambda\right) \gg M_{i}\left(d_{s} k_{\theta} \Lambda\right)$ for any $i=1, \ldots, 2 d$. The claim follows now from (4.4). 


\subsection{Irrational and diophantine lattices}

The purpose of this section is to relate the $\alpha_{d}$-characteristic of $\Lambda_{t}$ to the Diophantine approximation of $t Q$ by symmetric integral matrices. We begin by motivating the Definition 1.6: Recall that $Q$ is said to be Diophantine of type $(\kappa, A)$, where $\kappa>0$ and $A>0$, if

$$
\inf _{t \in[1,2]}\|M-m t Q\|>A m^{-\kappa} \text { for all } m \in \mathbb{Z} \backslash\{0\} \text { and } M \in \operatorname{Sym}(d, \mathbb{Z})
$$

or equivalently if we introduce the truncated rational approximation error

$$
\delta_{t} Q ; R \stackrel{\text { def }}{=} \min \{\|M-m t Q\|: m \in \mathbb{Z}, 0<|m| \leq R, M \in \operatorname{Sym}(d, \mathbb{Z})\}, \quad R \geq 1,
$$

we require $Q$ to satisfy

$$
\inf _{t \in[1,2]} \delta_{t Q ; R}>A R^{-\kappa} \text { for all } R \geq 1 .
$$

Remark 4.8 As an aside, we remark that the property of $Q$ being Diophantine in the above sense is equivalent to the requirement that for some $\tilde{\kappa}>0$

$$
\|M-t Q\|>t^{-\tilde{\kappa}}, \quad \text { for all } t \geq 2 \text { and } M \in \operatorname{Sym}(d, \mathbb{Z}),
$$

which was introduced in [23] in the context of forms that are (EWAS). However, this formulation is not optimal because $\tilde{\kappa}$ must be chosen larger than $\kappa$ depending on $A$. Moreover, in most applications the constant $A$ cannot be determined explicitly due to non-effective methods in Diophantine approximation.

The following lemma justifies calling such forms Diophantine:

Lemma 4.9 Let $k$ be an integer in the range $1 \leq k \leq \frac{d(d+1)}{2}-1$ and let $Q$ be a form such that $k+1$ non-zero entries $y, x_{1}, \ldots, x_{k}$ satisfy the property that

$$
\max _{i=1, \ldots, k}\left|q x_{i} / y+p_{i}\right|>A q^{-\kappa}
$$

for all $k$-tuples $\left(p_{1} / q, \ldots, p_{k} / q\right)$ of rationals. Then $Q$ is Diophantine of type ( $\left.\kappa, A^{\prime}\right)$, where $A^{\prime}$ depends on $A, \kappa, y, x_{1} / y, \ldots, x_{k} / y$ only (see (4.36)).

Proof Let $M \in \operatorname{Sym}(d, \mathbb{Z}), m \in \mathbb{Z} \backslash\{0\}$ and $t \in[1,2]$. Denoting the entries in $M$ corresponding to the coordinates of $Q$ in which $y, x_{1}, \ldots, x_{k}$ appear by $q, p_{1}, \ldots, p_{k}$, we find the inequality

$$
\|M-m t Q\| \geq \max \left\{\max _{1 \leq i \leq k}\left|p_{i}-m t x_{i}\right|,|q-m t y|\right\} .
$$


Suppose that the expression on the right-hand side is strictly less than $A^{\prime} m^{-\kappa}$, where

$$
\left.A^{\prime}=\min \left\{A(4 y)^{-\kappa}\left(1+\max _{1 \leq i \leq k}\left|x_{i} / y\right|\right)\right)^{-1}, 1 / 2\right\} .
$$

Note first that $|m| \geq|m t y| /(2 y)>q /(4 y)$ and hence

$\left|\frac{x_{i}}{y} q-p_{i}\right| \leq\left|\frac{x_{i}}{y}\right||q-m t y|+\left|m t x_{i}-p_{i}\right|<A^{\prime} m^{-\kappa}\left(1+\left|x_{i} / y\right|\right)<A q^{-\kappa}$

for all $i=1, \ldots, k$, which yields a contradiction.

Recall that a number $\theta \in \mathbb{R}$ is called Diophantine of type $\kappa>0$ if there exists $c_{\kappa}>0$ such that $|q \theta-a| \geq c_{\kappa}|q|^{-\kappa}$ for every rational number $a / q$. In particular any form $Q$ for which one ratio of two of its entries is a Diophantine number, is Diophantine in the sense of Definition 1.6 and hence almost all forms are Diophantine in this sense. An example of Diophantine forms for which we can control the exponent $\kappa$ is the following: Suppose $Q$ is a form with $k+1$ entries $y, x_{1}, \ldots, x_{k}$ such that $x_{1} / y, \ldots, x_{k} / y$ are algebraic and $1, x_{1} / y, \ldots, x_{k} / y$ are linearly independent over $\mathbb{Q}$, then Schmidt's Subspace Theorem together with Lemma 4.9 implies that for any $\eta>0$ the form $Q$ is Diophantine of type $\left(1 / k+\eta, A^{\prime}\right)$, where $A^{\prime}$ is a constant depending only on $\eta, A, y, x_{1} / y, \ldots, x_{k} / y$. However, as is usually the case in Diophantine approximation, the constant $A$ and hence $A^{\prime}$ is ineffective in the sense that these constants cannot be determined explicitly.

After the previous motivation, we shall state the main result of this section. In particular, we will see that larger values of $\beta_{t ; r}$ (see (4.38)) enforce smaller values of the truncated rational approximation error $\delta_{4 t} Q ; R$ as follows

Lemma 4.10 Assume that $q_{0} \geq 1$. Then we have for all $t \in \mathbb{R}$ and $r \geq q^{1 / 2}$

$$
\delta_{4 t Q ; \beta_{t ; r}^{-1}} \ll_{d} q r^{-2} \beta_{t ; r}^{-1},
$$

where

$$
\beta_{t ; r} \stackrel{\text { def }}{=} \alpha_{d}\left(\Lambda_{t}\right) r^{-d}|\operatorname{det} Q|^{1 / 2}
$$

Note that this bound is non-trivial for $\beta_{t ; r}>q r^{-2}$ only, due to the uniform bound $\beta_{t ; r} \ll_{d} 1$ for $r \geq q^{1 / 2}$ established in Lemma 4.6.

Before proving (4.37), we shall state some important consequences.

Corollary 4.11 Consider any interval $\left[T_{-}, T_{+}\right]$with $T_{-} \in(0,1]$ and $T_{+} \geq 1$. 
(i) If $Q$ is irrational, then

$$
\lim _{r \rightarrow \infty}\left(\sup _{T_{-} \leq t \leq T_{+}} \alpha_{d}\left(\Lambda_{t}\right) r^{-d}\right)=0 .
$$

(ii) If $Q$ is Diophantine of type $(\kappa, A)$, then

$$
\sup _{T_{-} \leq t \leq T_{+}} \alpha_{d}\left(\Lambda_{t}\right) r^{-d} \ll_{d}|\operatorname{det} Q|^{-1 / 2}\left(q A^{-1} r^{-2}\right)^{\frac{1}{k+1}} \max \left\{\left(T_{-}\right)^{-\frac{1}{k+1}},\left(T_{+}\right)^{\frac{k}{k+1}}\right\} .
$$

A variant of (i) in terms of the successive minima of $\Lambda_{t}$ can also be found in [28], see Lemma 3.11, yielding an alternative proof of (4.39) when combined with (4.4).

Proof (i) We show the contraposition: Assume that there exists an $\varepsilon>0$ and sequences $\left(r_{j}\right)_{j},\left(t_{j}\right)_{j}$ such that $\lim _{j \rightarrow \infty} r_{j}=\infty$ and $\beta_{t_{j} ; r_{j}}>\varepsilon$. Passing to a subsequence we may assume that $\lim _{j \rightarrow \infty} t_{j}=t$ for some $t \in\left[T_{-}, T_{+}\right]$. Thus (4.37) yields $\lim _{j \rightarrow \infty} \delta_{4 t_{j} Q ; R_{j}^{*}}=0$ with $R_{j}^{*}:=\beta_{t_{j} ; r_{j}}^{-1}<\varepsilon^{-1}$. By definition, this means that $\lim _{j \rightarrow \infty}\left\|M_{j}-4 t_{j} m_{j} Q\right\|=0$ for some $M_{j} \in \operatorname{Sym}(d, \mathbb{Z})$ and $m_{j} \in \mathbb{Z}$ with $\left|m_{j}\right| \leq \varepsilon^{-1}$. Obviously both, $\left\|M_{j}\right\|$ and $\left|m_{j}\right|$, are bounded. Hence there exist integral elements $M, m$ and an infinite subsequence $j^{\prime}$ of $j$ with $M_{j^{\prime}}=M, m_{j^{\prime}}=m$ and by construction $\lim _{j^{\prime}} t_{j^{\prime}}=t$. These limit values satisfy $\|M-4 m t Q\|=0$, i.e. $Q$ is a multiple of a rational form.

(ii) First we note that for any $t \in\left[1, T_{+}\right]$we have by (4.35)

$$
\left(\delta_{t Q ; R}\right)^{-1} \leq \sup _{t^{\prime} \in[1,2]}\left(\delta_{t^{\prime}} Q ; 4 t R\right)^{-1}<A^{-1}(4 t R)^{\kappa} \leq A^{-1}\left(T_{+}\right)^{\kappa}(4 R)^{\kappa}
$$

and similarly for $t \in\left[T_{-}, 1\right]$

$$
\left(T_{-}\right)^{-1} \delta_{4 t} Q ; R \gg\left\lceil t^{-1}\right\rceil \delta_{t} Q ; 4 R \geq \delta_{\left(\left\lceil t^{-1}\right\rceil t\right) Q ; 4 R}>A(4 R)^{-\kappa} .
$$

Thus, the relation (4.37), established in Lemma 4.10, implies for any $t \in$ $\left[T_{-}, T_{+}\right]$that

$$
\beta_{t ; r} \ll_{d} q r^{-2}\left(\delta_{4 t Q ; \beta_{t ; r}^{-1}}\right)^{-1} \ll_{d} 4^{\kappa} q r^{-2} A^{-1} \max \left\{\left(T_{-}\right)^{-1},\left(T_{+}\right)^{\kappa}\right\}\left(\beta_{t ; r}\right)^{-\kappa},
$$

where we used (4.37). Therefore we conclude (4.40) as claimed.

Proof of Lemma 4.10 We begin by recalling that $\Lambda_{t}=D_{r Q} U_{4 t} Q \mathbb{Z}^{2 d}$ (see (3.47)), where

$$
D_{r Q}=\left(\begin{array}{cc}
r Q_{+}^{-1 / 2} & 0 \\
0 & r^{-1} Q_{+}^{1 / 2}
\end{array}\right) \quad \text { and } \quad U_{4 t Q}=\left(\begin{array}{cc}
\mathrm{I}_{d}-4 t Q \\
0 & \mathrm{I}_{d}
\end{array}\right) .
$$


As noted in Remark 2.1 the $\alpha_{d}$-characteristic of $\Lambda_{t}$ is attained at some sublattice, that is we can write $\alpha_{d}\left(\Lambda_{t}\right)=\left\|w_{1} \wedge \ldots \wedge w_{d}\right\|^{-1}$ by means of vectors $w_{j}:=D_{r Q} U_{4 t} l_{j}$ with linear independent points $l_{1}, \ldots, l_{d} \in \mathbb{Z}^{2 d}$ depending on $t$. Here we use the standard Euclidean norm on the exterior product $\wedge^{d} \mathbb{R}^{2 d}$. Moreover, we write $l_{j}=\left(m_{j}, n_{j}\right)$, where $m_{j}, n_{j} \in \mathbb{Z}^{d}$ and the coordinates of $\left(m_{j}, n_{j}\right)$ are the coordinates of the vectors $m_{j}$ and $n_{j}$ in the corresponding order. Additionally, we introduce the $d \times d$ integer matrices $N$ and $M$ with columns $n_{1}, \ldots, n_{d}$ and $m_{1}, \ldots, m_{d}$ as well. Using this notation, we may write

$$
w_{1} \wedge \ldots \wedge w_{d}=\left(D_{r Q} U_{4 t}\right)\left(\begin{array}{c}
M \\
N
\end{array}\right) e_{1} \wedge \ldots \wedge e_{d}
$$

First, we shall prove that

$$
\alpha_{d}\left(\Lambda_{t}\right)>q d_{Q} r^{d-2} \quad \text { implies } \quad \beta_{t ; r}^{-1}>|\operatorname{det}(N)|>0 .
$$

Note that the left-hand side of (4.42) can be rewritten as $\beta_{t ; r}>q r^{-2}$ and we may assume that this inequality holds, since otherwise the bound (4.37) is trivial.

Let us show that $\operatorname{rank}(N)=d$. To this end, we write $k=d-\operatorname{rank}(N)$. According to elementary divisor theory (for matrices with entries in a principal ideal domain) there exist $P, P^{\prime} \in \mathrm{GL}(d, \mathbb{Z})$ such that $P^{\prime} N P$ is a diagonal matrix with positive entries of the form $\operatorname{diag}\left(0, \ldots, 0, a_{k+1}, \ldots, a_{d}\right)$ with $a_{i}$ | $a_{i+1}, a_{i} \in \mathbb{N}$. In particular $N P$ is a matrix whose first $k$ columns are zero. Moreover, since det $P= \pm 1$, we conclude that

$$
\left(\begin{array}{l}
M P \\
N P
\end{array}\right) e_{1} \wedge \ldots \wedge e_{d}= \pm\left(\begin{array}{c}
M \\
N
\end{array}\right) e_{1} \wedge \ldots \wedge e_{d}
$$

and hence we can assume from now on that $N=\left(0, \ldots, 0, n_{k+1}, \ldots, n_{d}\right)$ with linearly independent vectors $n_{k+1}, \ldots, n_{d} \in \mathbb{Z}^{d}$. Since $l_{1}, \ldots, l_{d}$ constitute a basis of a $d$-dimensional lattice, we note that $m_{1}, \ldots, m_{k}$ are necessarily linearly independent. Now we shall express $w_{1} \wedge \ldots \wedge w_{d}$ in terms of the standard basis $e_{I} \wedge e_{J}$ indexed by pairs of subsets $I \subset\{1, \ldots, d\}$ and $J \subset$ $\{d+1, \ldots, 2 d\}$ with $|I|+|J|=d$, i.e. we write

$$
w_{1} \wedge \ldots \wedge w_{d}=\sum_{I, J} \omega_{I, J} e_{I} \wedge e_{J}
$$


Let $I=\left\{i_{1}, \ldots, i_{m}\right\}$ and $J=\left\{j_{1}, \ldots, j_{d-m}\right\}$, then the coefficients $\omega_{I, J}$ are given by

$$
\omega_{I, J} \stackrel{\text { def }}{=} \operatorname{det}\left(\begin{array}{cc}
A_{I} & * \\
0 & B_{J}
\end{array}\right)
$$

where

$$
\begin{aligned}
A_{I} \stackrel{\text { def }}{=}\left(\begin{array}{ccc}
\left\langle r Q_{+}^{-\frac{1}{2}} m_{1}, e_{i_{1}}\right\rangle & \ldots & \left\langle r Q_{+}^{-\frac{1}{2}} m_{k}, e_{i_{1}}\right\rangle \\
\vdots & \vdots \\
\left\langle r Q_{+}^{-\frac{1}{2}} m_{1}, e_{i_{m}}\right\rangle & \ldots & \left\langle r Q_{+}^{-\frac{1}{2}} m_{k}, e_{i_{m}}\right\rangle
\end{array}\right) \\
B_{J} \stackrel{\text { def }}{=}\left(\begin{array}{ccc}
\left\langle r^{-1} Q_{+}^{\frac{1}{2}} n_{k+1}, e_{j_{1}}\right\rangle & \ldots & \left\langle r^{-1} Q_{+}^{\frac{1}{2}} n_{d}, e_{j_{1}}\right\rangle \\
\vdots & \vdots \\
\left\langle r^{-1} Q_{+}^{\frac{1}{2}} n_{k+1}, e_{j_{d-m}}\right\rangle & \ldots & \left\langle r^{-1} Q_{+}^{\frac{1}{2}} n_{d}, e_{j_{d-m}}\right\rangle
\end{array}\right) .
\end{aligned}
$$

Since the matrix in (4.43) is of block-type, we find

$$
\begin{aligned}
\alpha_{d}\left(\Lambda_{t}\right)^{-2} & =\left\|w_{1} \wedge \ldots \wedge w_{d}\right\|^{2} \\
& \geq \sum_{|I|=k} \sum_{|J|=d-k} \omega_{I, J}^{2}=\left(\sum_{|I|=k}\left(\operatorname{det} A_{I}\right)^{2}\right)\left(\sum_{|J|=d-k}\left(\operatorname{det} B_{J}\right)^{2}\right) \\
& =r^{4 k-2 d}\left\|Q_{+}^{-\frac{1}{2}}\left(m_{1} \wedge \ldots \wedge m_{k}\right)\right\|^{2}\left\|Q_{+}^{\frac{1}{2}}\left(n_{k+1} \wedge \ldots \wedge n_{d}\right)\right\|^{2} .
\end{aligned}
$$

Without loss of generality assume that the eigenvalues of $Q$ are indexed such that $\left|q_{1}\right| \leq \cdots \leq\left|q_{d}\right|$. Since $q_{0} \geq 1$, note that the minimal eigenvalue of the $k$-th exterior power of $Q_{+}^{-1 / 2}$ is given by $\left|q_{d-k+1} \ldots q_{d}\right|^{-1 / 2}$ and that of the $(d-k)$-th exterior power of $Q_{+}^{1 / 2}$ is precisely $\left|q_{1} \ldots q_{d-k}\right|^{1 / 2}$. Hence, since $m_{1}, \ldots, m_{k}$ and $n_{k+1}, \ldots, n_{d}$ are linearly independent and integral, we obtain the following lower bound

$$
\alpha_{d}\left(\Lambda_{t}\right)^{-1} \geq r^{2 k-d}\left(\frac{\left|q_{1} \ldots q_{d-k}\right|}{\left|q_{d-k+1} \ldots q_{d}\right|}\right)^{1 / 2} \geq q^{-1}|\operatorname{det} Q|^{1 / 2} r^{2-d}
$$

where we used that $r \geq q^{1 / 2}$. In view of (4.42), this strict inequality yields a contradiction unless $k=0$. Thus, we proved that $k=0$, i.e. $|\operatorname{det} N|>0$. Now (4.44) also implies $\beta_{t ; r}^{-1} \geq|\operatorname{det} N|$. Hence, the upper bound for $|\operatorname{det} N|$ in (4.42) holds as well. 
Finally, we shall prove (4.37). Since $N$ is invertible, we can rewrite $w_{1} \wedge$ $\ldots \wedge w_{d}$ by

$$
\left(D_{r Q} U_{4 t Q}\right)\left(\begin{array}{c}
M N^{-1} \\
\mathbb{1}_{d}
\end{array}\right) N\left(e_{1} \wedge \ldots \wedge e_{d}\right)=(\operatorname{det} N)\left(D_{r Q} U_{4 t} Q\right)\left(\begin{array}{c}
M N^{-1} \\
\mathbb{1}_{d}
\end{array}\right) e_{1} \wedge \ldots \wedge e_{d},
$$

i.e. we parametrized the subspace spanned by $l_{1}, \ldots, l_{d}$. Introduce also the $2 d \times d$ matrix

$$
W \stackrel{\text { def }}{=}\left(D_{r Q} U_{4 t Q}\right)\left(\begin{array}{c}
M N^{-1} \\
\mathbb{1}_{d}
\end{array}\right)=\left(\begin{array}{c}
r Q_{+}^{-\frac{1}{2}}\left(M N^{-1}-4 t Q\right) \\
r^{-1} Q_{+}^{\frac{1}{2}}
\end{array}\right)
$$

and note that $W^{T} W$ is a positive definite symmetric $d \times d$ matrix. Thus, there exists an orthogonal matrix $V \in O(d)$ such that $D:=V^{T} W^{T} W V$ is diagonal with positive entries. Since $(\operatorname{det} V)\left(e_{1} \wedge \ldots \wedge e_{d}\right)=V\left(e_{1} \wedge \ldots \wedge e_{d}\right)$ it follows that

$$
\begin{aligned}
\| W\left(e_{1} \wedge\right. & \left.\ldots \wedge e_{d}\right)\left\|^{2}=\right\| W V\left(e_{1} \wedge \ldots \wedge e_{d}\right) \|^{2} \\
& =\left\langle D\left(e_{1} \wedge \ldots \wedge e_{d}\right),\left(e_{1} \wedge \ldots \wedge e_{d}\right)\right\rangle=\prod_{i=1}^{d}\left\|D e_{i}\right\|=\prod_{i=1}^{d}\left\|W v_{i}\right\|^{2},
\end{aligned}
$$

where $v_{1}, \ldots, v_{d}$ denote the columns of $V$. Next observe that

$$
\max _{1 \leq i \leq d}\left\|W v_{i}\right\| \geq \max _{1 \leq i \leq d}\left\|r Q_{+}^{-\frac{1}{2}}\left(M N^{-1}-4 t Q\right) v_{i}\right\| \gg_{d} r q^{-\frac{1}{2}}\left\|M N^{-1}-4 t Q\right\| .
$$

Now let $i_{0}$ be a subscript for which $\left\|W v_{i}\right\|$ is maximal. Similar to the proof of (4.44) we may write $W\left(\wedge_{i \neq i_{0}} v_{i}\right)=\sum \omega_{I, J} e_{I} \wedge e_{J}$, where the sum is taken over subsets $I \subset\{1, \ldots, d\}$ and $J \subset\{d+1, \ldots, 2 d\}$ with $|I|+|J|=d-1$, and find that

$$
\left\|W\left(\wedge_{i \neq i_{0}} v_{i}\right)\right\|^{2} \geq \sum_{|I|=0,|J|=d-1} \omega_{I, J}^{2}=\left\|r^{-1} Q_{+}^{\frac{1}{2}}\left(\wedge_{i \neq i_{0}} v_{i}\right)\right\|^{2} \geq r^{-2(d-1)} q^{-1}|\operatorname{det} Q| .
$$

Combining (4.45) together with (4.46)-(4.48) yields

$$
\begin{aligned}
\alpha_{d}\left(\Lambda_{t}\right)^{-1} & =|\operatorname{det}(N)|\left\|W v_{i_{0}}\right\| \prod_{i \neq i_{0}}\left\|W v_{i}\right\| \\
& =|\operatorname{det}(N)|\left\|W v_{i_{0}}\right\|\left\|W\left(\wedge_{i \neq i_{0}} v_{i}\right)\right\| \\
& \gg_{d} r^{-(d-2)} q^{-1}|\operatorname{det} Q|^{\frac{1}{2}}|\operatorname{det} N|\left\|M N^{-1}-4 t Q\right\| .
\end{aligned}
$$


Since $(\operatorname{det} N) N^{-1}$ is an integral matrix, the last line together with (4.42) implies

$$
\min \left\{\|\bar{M}-4 m t Q\|: 0<|m| \leq \beta_{t ; r}^{-1}, m, \bar{M} \text { integral }\right\} \ll_{d} q r^{-2} \beta_{t ; r}^{-1},
$$

and, since $Q$ is symmetric, we may take $\bar{M}$ symmetric as well, which proves (4.37).

\section{Averages along translates of orbits of $\mathrm{SO}(2)$}

\subsection{Application of geometry of numbers}

In view of the bound (3.39) we need to estimate the error term $I_{\theta}$, that is (3.22). Proceeding as in (3.23) combined with the estimates $\left|\theta_{v}(t)\right| \ll_{d}$ $|\operatorname{det} Q|^{-1 / 4} r^{d / 2} \psi(r, t)^{1 / 2}$ and $\psi(r, t) \ll_{d} \alpha_{d}\left(\Lambda_{t}\right)$, obtained in Lemma 3.3 respectively (4.14) of Corollary 4.5, leads to

$$
I_{\theta} \ll_{d} r^{d / 2}|\operatorname{det} Q|^{-1 / 4}\|\widehat{\zeta}\|_{1} \int_{|t|>q_{0}^{-1 / 2} r^{-1}}\left|\widehat{g}_{w}(t)\right| \alpha_{d}\left(\Lambda_{t}\right)^{1 / 2} \mathrm{~d} t
$$

where $\Lambda_{t}$ denotes the lattice defined in (3.47) and $g_{w}$ the smoothed indicator function of $[a, b]$ with $0<w<(b-a) / 4$, see Corollary 3.2. Since Lemma 7.2 provides estimates for $\|\widehat{\zeta}\|_{1}$ in the case of both admissible and non-admissible regions $\Omega$, it remains to estimate the integral in (5.1). We shall start with bounding this integral over an interval $I$ of length at most $1 / q$. For this, we introduce the maximum value over $I$ of the $\alpha_{d}$-characteristic for the lattice $\Lambda_{t}$ via

$$
\gamma_{I, \beta}(r) \stackrel{\text { def }}{=} \sup \left\{\left(r^{-d} \alpha_{d}\left(\Lambda_{t}\right)\right)^{\frac{1}{2}-\beta}: t \in I\right\}
$$

and the following family of lattices

$$
\Lambda_{Q, t}:=d_{q^{1 / 2}} u_{4 t} \Lambda_{Q},
$$

where $\Lambda_{Q}$ is as defined in (4.29). Here $\gamma_{I, \beta}(r)$ depends on the Diophantine properties of $Q$ and tends to zero for growing $r \rightarrow \infty$ by Lemma 4.11 for irrational $Q$.

Lemma 5.1 Let $r \geq q^{1 / 2}, 0<\beta \leq 1 / 2$ and fix an interval $I=\left[\tau_{1}, \tau_{2}\right]$ of length at most $1 / q$. Then we have

$$
\int_{I} \alpha_{d}\left(\Lambda_{t}\right)^{1 / 2}\left|\widehat{g}_{w}(t)\right| \mathrm{d} t \ll_{d} \widehat{g}_{I} r^{\frac{d}{2}-\beta d} \gamma_{I, \beta}(r) \frac{1}{q} \int_{-\pi}^{\pi} \alpha\left(d_{r_{*}} k_{\theta} \Lambda_{Q, 4 \tau_{1}}\right)^{\beta} \frac{\mathrm{d} \theta}{2 \pi},
$$


where $r_{*}:=r q^{-1 / 2}$ and $\widehat{g}_{I}:=\max \left\{\left|\widehat{g}_{w}(t)\right|: t \in I\right\}$.

Proof Using the trivial bound $\alpha_{d}\left(\Lambda_{t}\right) \leq r^{d-2 \beta d} \gamma_{I, \beta}(r)^{2} \alpha_{d}\left(\Lambda_{t}\right)^{2 \beta}$ and estimating $\left|\widehat{g}_{w}\right|$ by its maximum $\widehat{g}_{I}$ on $I$ yields

$$
\int_{I} \alpha_{d}\left(\Lambda_{t}\right)^{1 / 2}\left|\widehat{g}_{w}(t)\right| \mathrm{d} t \leq \widehat{g}_{I} r^{\frac{d}{2}-d \beta} \gamma_{I, \beta}(r) \int_{I} \alpha_{d}\left(\Lambda_{t}\right)^{\beta} \mathrm{d} t .
$$

Since the group $\mathrm{D}$ normalizes $\mathrm{U}$, a computation shows that $d_{r} u_{4 t}=$ $d_{r} u_{4\left(t-\tau_{1}\right)} u_{4 \tau_{1}}=d_{r_{*}} u_{\tau} d_{q^{1 / 2}} u_{4 \tau_{1}}$, where $\tau:=4\left(t-\tau_{1}\right) q$. Changing variables from $t$ to $\tau$ we obtain in terms of the lattices $\Lambda_{Q, s}$, defined in (5.3),

$$
\int_{I} \alpha_{d}\left(\Lambda_{t}\right)^{\beta} \mathrm{d} t=\int_{\tau_{1}}^{\tau_{2}} \alpha_{d}\left(d_{r_{*}} u_{\tau} d_{q^{1 / 2}} u_{4 \tau_{1}} \Lambda_{Q}\right)^{\beta} \mathrm{d} t \ll \frac{1}{q} \int_{0}^{4} \alpha_{d}\left(d_{r_{*}} u_{\tau} \Lambda_{Q, 4 \tau_{1}}\right)^{\beta} \mathrm{d} \tau .
$$

Finally, we estimate the last average with the help of Lemma 4.7 by the average over the group $\mathrm{K}=\mathrm{SO}(2)$. Changing variables $\theta(s)=\arctan (\tau), \tau \in[0,4]$, and noting that $|\theta|<\pi$ and $\mathrm{d} \tau=\left(1+\tau^{2}\right) \mathrm{d} \theta$, we get by (4.31) of Lemma 4.7 that

$$
\begin{aligned}
\int_{0}^{4} \alpha_{d}\left(d_{r_{*}} u_{\tau} \Lambda_{Q, 4 \tau_{1}}\right)^{\beta} \mathrm{d} \tau & \ll \int_{0}^{4} \alpha_{d}\left(d_{r_{*}} k_{\theta(\tau)} \Lambda_{Q, 4 \tau_{1}}\right)^{\beta} \mathrm{d} \tau \\
& \ll \int_{-\pi}^{\pi} \alpha_{d}\left(d_{r_{*}} k_{\theta} \Lambda_{Q, 4 \tau_{1}}\right)^{\beta} \frac{\mathrm{d} \theta}{2 \pi} .
\end{aligned}
$$

Now note that $\alpha_{d}(\Lambda) \leq \alpha(\Lambda)$ holds for any lattice $\Lambda$ in $\mathbb{R}^{2 d}$. Thus, the last inequality together with (5.5) and (5.6) completes the proof.

In the following paragraphs we shall develop explicit bounds for averages over the group K of type $\int_{\mathrm{K}} \alpha_{d}\left(d_{r} k \Lambda\right)^{\beta} \mathrm{d} k$.

\subsection{Operators $A_{g}$ and functions $\tau_{\lambda}$ on $\operatorname{SL}(2, \mathbb{R})$}

Let $\mathrm{G}=\mathrm{SL}(2, \mathbb{R})$. We consider the following two subgroups of $\mathrm{G}$ :

$$
\mathrm{K}=\mathrm{SO}(2)=\left\{k_{\theta}: 0 \leq \theta<2 \pi\right\} \quad \text { and } \mathrm{T}=\left\{\left(\begin{array}{cc}
a & b \\
0 & a^{-1}
\end{array}\right): a>0, b \in \mathbb{R}\right\} \text {, }
$$

where $k_{\theta}$ is defined in (4.26). According to the Iwasawa decomposition, any $g \in G$ can be uniquely represented as a product of elements from $\mathrm{K}$ and $\mathrm{T}$, that is

$$
g=k(g) t(g), \quad k(g) \in \mathrm{K}, t(g) \in \mathrm{T} .
$$


Now let

$$
d_{a} \stackrel{\text { def }}{=}\left(\begin{array}{cc}
a & 0 \\
0 & a^{-1}
\end{array}\right) \text { for } a>0 \text { and } \mathrm{D}^{+}=\left\{d_{a}: a \geq 1\right\}
$$

According to the Cartan decomposition, we have

$$
\mathrm{G}=\mathrm{KD}^{+} \mathrm{K}, \quad g=k_{1}(g) d(g) k_{2}(g), g \in \mathrm{G}, k_{1}(g), k_{2}(g) \in \mathrm{K}, d(g) \in \mathrm{D}^{+} .
$$

In this decomposition $d(g)$ is determined by $g$, and if $g \notin \mathrm{K}$ then $k_{1}(g)$ and $k_{2}(g)$ are also determined by $g$ up to a factor of \pm 1 on $k_{1}$ and $k_{2}$. It is clear that $\|g\|=\|d(g)\|$, where $\|\cdot\|$ denotes the operator norm induced by the standard Euclidean norm on $\mathbb{R}^{2}$. Note that, in the simple case $g=d_{a}$, this norm is given by $\left\|d_{a}\right\|=a$. Since $d_{a}$ is the conjugate of $d_{a^{-1}}$ by $k_{\pi / 2}$, we see that $g^{-1} \in \mathrm{KgK}$ or equivalently, $d(g)=d\left(g^{-1}\right)$ for any $g \in \mathrm{G}$. Therefore, $\|g\|=\left\|g^{-1}\right\|, g \in \mathrm{G}$.

We say that a function $f$ on $\mathrm{G}$ is left $\mathrm{K}$-invariant (resp. right $\mathrm{K}$-invariant, resp. bi-K-invariant) if $f(\mathrm{~K} g)=f(g)$ (resp. $f(g \mathrm{~K})=f(g)$, resp. $f(\mathrm{~K} g \mathrm{~K})=f(g))$. Any bi-K-invariant function on $\mathrm{G}$ is completely determined by its restriction to $\mathrm{D}^{+}$. Hence for any bi-K-invariant function $f$ on $\mathrm{G}$, there is a function $f^{*}$ on $[1, \infty)$ such that $f(g)=f^{*}(\|g\|), g \in \mathrm{G}$.

For any $\lambda \in \mathbb{R}$ we define a character $\chi_{\lambda}$ of T by

$$
\chi_{\lambda}\left(\begin{array}{cc}
a & b \\
0 & a^{-1}
\end{array}\right)=a^{-\lambda}
$$

and the function $\varphi_{\lambda}: \mathrm{G} \rightarrow \mathbb{R}^{+}$by

$$
\varphi_{\lambda}(g)=\chi_{\lambda}(t(g)), \quad g \in G
$$

The function $\varphi_{\lambda}$ has the property

$$
\varphi_{\lambda}(k g t)=\chi_{\lambda}(t) \varphi_{\lambda}(g), \quad g \in \mathrm{G}, k \in \mathrm{K}, t \in \mathrm{T}
$$

and it is completely determined by this property and the condition $\varphi_{\lambda}(1)=1$.

For $g \in \mathrm{G}$ and a continuous action of $\mathrm{G}$ on a topological space $X$, we define the operator $A_{g}$ on the space of continuous functions on $X$ by

$$
\left(A_{g} f\right)(x)=\int_{\mathrm{K}} f(g k x) \mathrm{d} \sigma(k), \quad x \in X,
$$


where $\sigma$ is the normalized Haar measure on $\mathrm{K}$, or, using the parametrization of $\mathrm{K}$, by

$$
\left(A_{g} f\right)(x)=\frac{1}{2 \pi} \int_{0}^{2 \pi} f\left(g k_{\theta} x\right) \mathrm{d} \theta, \quad x \in X .
$$

The operator $A_{g}$ is a linear map into the space of left $\mathrm{K}$-invariant functions on $X$. If $X=\mathrm{G}$ and $\mathrm{G}$ acts on itself by left translations, then $A_{g}$ commutes with right translations. From these two remarks, or using a direct computation, we get that $A_{g} \varphi_{\lambda}$ has the property (5.7). Hence $\varphi_{\lambda}$ is an eigenfunction for $A_{g}$ with the eigenvalue

$$
\tau_{\lambda}(g) \stackrel{\text { def }}{=}\left(A_{g} \varphi_{\lambda}\right)(1)=\int_{\mathrm{K}} \varphi_{\lambda}(g k) \mathrm{d} \sigma(k)=\int_{\mathrm{K}} \chi_{\lambda}(t(g k)) \mathrm{d} \sigma(k) .
$$

We see from (5.9) that $\tau_{\lambda}$ is obtained from $\varphi_{\lambda}$ by averaging over right translations by elements of $\mathrm{K}$. But $\varphi_{\lambda}$ is left $\mathrm{K}$-invariant and $A_{g}$ commutes with right translations. Hence the function $\tau_{\lambda}$ is bi-K-invariant and it is an eigenfunction for $A_{g}$ with the eigenvalue $\tau_{\lambda}(g)$, that is

$$
\left(A_{g} \tau_{\lambda}\right)(h)=\tau_{\lambda}(g) \tau_{\lambda}(h) \text { for all } h \in \mathrm{G} \text {. }
$$

We have that

$$
\varphi_{\lambda}(g)=\left\|g e_{1}\right\|^{-\lambda}, \quad g \in G, e_{1}=(1,0)
$$

where $\|\cdot\|$ denotes the usual Euclidean norm on $\mathbb{R}^{2}$. Indeed

$$
\varphi_{\lambda}(g)=\chi_{\lambda}(t(g))=\left\|t(g) e_{1}\right\|^{-\lambda}=\left\|k(g) t(g) e_{1}\right\|^{-\lambda}=\left\|g e_{1}\right\|^{-\lambda} .
$$

From (5.9) and (5.11) we get

$$
\begin{aligned}
\tau_{\lambda}(g) & =\int_{K}\left\|g k e_{1}\right\|^{-\lambda} \mathrm{d} \sigma(k)=\frac{1}{2 \pi} \int_{0}^{2 \pi}\left\|g k(\theta) e_{1}\right\|^{-\lambda} \mathrm{d} \theta \\
& =\frac{1}{2 \pi} \int_{0}^{2 \pi}\|g(\cos \theta, \sin \theta)\|^{-\lambda} \mathrm{d} \theta=\int_{S^{1}}\|g u\|^{-\lambda} \mathrm{d} \ell(u),
\end{aligned}
$$

where $S^{1}$ is the unit circle in $\mathbb{R}^{2}$ and $\ell$ denotes the normalized rotation invariant measure on $S^{1}$. One can easily see that $\|g u\|^{-2}, g \in G, u \in S^{1}$, is equal to the Jacobian at $u$ of the diffeomorphism $v \mapsto g v /\|g v\|$ of $S^{1}$ onto $S^{1}$. On the other hand, it follows from the change of variables formula that

$$
\int_{M} J_{f}^{\lambda}=\int_{M} J_{f^{-1}}^{1-\lambda}, \quad \lambda \in \mathbb{R},
$$


where $f: M \rightarrow M$ is a diffeomorphism of a compact differentiable manifold $M$ and $J_{f}$ (resp. $J_{f^{-1}}$ ) denotes the Jacobian of $f\left(\right.$ resp. $f^{-1}$ ). Now using (5.12) we get

$$
\tau_{\lambda}(g)=\tau_{2-\lambda}\left(g^{-1}\right)=\tau_{2-\lambda}(g), g \in G, \lambda \in \mathbb{R} .
$$

The second equality in (5.13) is true because $\tau_{\lambda}$ is bi-K-invariant and $g^{-1} \in$ $\mathrm{K} g \mathrm{~K}$. Since, obviously, $\tau_{0}(g)=1$, it follows that

$$
\tau_{2}(g)=\tau_{0}(g)=1 .
$$

Since $t^{-\lambda}$ is a strictly convex function of $\lambda$ for any $t>0, t \neq 1$, it follows from (5.12) that $\tau_{\lambda}(g)$ is a strictly convex function of $\lambda$ for any $g \in G$. From this, (5.13) and (5.14) we deduce that

$$
\begin{aligned}
\tau_{\eta}(g)<\tau_{\lambda}(g) & \text { for any } g \notin \mathrm{K} \text { and } 1 \leq \eta<\lambda \leq 2, \\
\tau_{\eta}(g)<1 \text { and } \tau_{\lambda}(g)>1 & \text { for any } g \notin \mathrm{K}, 0<\eta<2, \lambda>2, \text { and } \\
\tau_{\eta}(g)<\tau_{\lambda}(g) & \text { for any } g \notin \mathrm{K}, \lambda \geq 2,0<\eta<\lambda .
\end{aligned}
$$

Since the function $\tau_{\lambda}(g)$ is bi-K-invariant, it depends only on the norm $\|g\|$ of $g$. Thus, we can write

$$
\tau_{\lambda}(g)=\tau_{\lambda}^{*}(\|g\|), \quad g \in \mathrm{G},
$$

where for $a \geq 1$

$$
\tau_{\lambda}^{*}(a)=\tau_{\lambda}\left(d_{a}\right)=\int_{\mathrm{K}}\left\|d_{a} k e_{1}\right\|^{-\lambda} \mathrm{d} \sigma(k)=\frac{1}{2 \pi} \int_{0}^{2 \pi} \frac{\mathrm{d} \theta}{\left(a^{2} \cos ^{2} \theta+a^{-2} \sin ^{2} \theta\right)^{\lambda / 2}} .
$$

In view of (5.10) and the definition of $A_{g}$, we get

$$
\int_{K} \tau_{\lambda}^{*}\left(\left\|g k d_{a}\right\|\right) \mathrm{d} \sigma(k)=\tau_{\lambda}(g) \tau_{\lambda}^{*}(a), \quad g \in \mathrm{G}, a \geq 1
$$

Since $\|g\|=\left\|g^{-1}\right\|$ for all $g \in \mathrm{G}$,

$$
\frac{a}{\|g\|} \leq\left\|g k d_{a}\right\| \leq a\|g\|
$$

for all $k \in \mathrm{K}$ and $g \in \mathrm{G}$. From this, (5.15) and (5.19) we deduce that, for any $\lambda>2$, the continuous function $\tau_{\lambda}^{*}(a), a \geq 1$, does not have a local maximum. Hence $\tau_{\lambda}^{*}$ is strictly increasing for all $\lambda>2$ or, equivalently,

$$
\tau_{\lambda}(g)<\tau_{\lambda}(h) \text { if }\|g\|<\|h\|, g, h \in G, \lambda>2 .
$$


Using (5.13) and (5.18) yields

$$
\tau_{\lambda}^{*}(a)=\tau_{2-\lambda}^{*}(a)=\frac{1}{2 \pi} \int_{0}^{2 \pi}\left(a^{2} \cos ^{2} \theta+a^{-2} \sin ^{2} \theta\right)^{\frac{\lambda}{2}-1} \mathrm{~d} \theta .
$$

Since $a^{2} \cos ^{2} \theta \leq a^{2} \cos ^{2} \theta+a^{-2} \sin ^{2} \theta \leq a^{2}$, we deduce from (5.21) the estimates

$$
c(\lambda) a^{\lambda-2} \leq \hat{\tau}_{\lambda}(a) \leq a^{\lambda-2}, a \geq 1, \lambda \geq 2,
$$

where

$$
c(\lambda)=\frac{1}{2 \pi} \int_{0}^{2 \pi}|\cos \theta|^{\lambda-2} \mathrm{~d} \theta=\frac{2}{\pi} \int_{0}^{\pi / 2} \cos (\theta)^{\lambda-2} \mathrm{~d} \theta=\frac{\mathrm{B}\left(\frac{\lambda-1}{2}, \frac{1}{2}\right)}{\pi}=\frac{\Gamma\left(\frac{\lambda-1}{2}\right)}{\Gamma\left(\frac{\lambda}{2}\right) \sqrt{\pi}},
$$

$\mathrm{B}$ denotes the beta function and we use the identity $\mathrm{B}(x, y)=\Gamma(x) \Gamma(y) / \Gamma(x+$ $y$ ) as well as $\Gamma(1 / 2)=\sqrt{\pi}$. From (5.21) we also conclude that for any $\lambda>2$ the ratio $\frac{\tau_{\lambda}^{*}(a)}{a^{\lambda-2}}$ is a strictly decreasing function of $a \geq 1$ and

$$
\lim _{a \rightarrow \infty} \frac{\tau_{\lambda}^{*}(a)}{a^{\lambda-2}}=c(\lambda)
$$

Remark 5.2 The function $\tau_{\lambda}$ can be viewed as a spherical function on the upper-half plane $\mathbb{H}$ (see [29] Chapter IV Proposition 2.9) and all spherical functions on $\mathbb{H}$ are of this form for some $\lambda \in \mathbb{C}$. In particular, it is not difficult to see that $\tau_{\lambda}$ can also be represented as

$$
\tau_{\lambda}(g)=\frac{1}{2 \pi} \int_{0}^{2 \pi}(\cosh (2 \log \|g\|)+\sinh (2 \log \|g\|) \sin (\theta))^{\lambda / 2-1} \mathrm{~d} \theta .
$$

Moreover, for $\operatorname{Re}(\lambda)>1$ it is well-known that $c(\lambda)$, which is usually referred to as Harish-Chandra's $c$-function, as defined in (5.24) exists and its value is given by (5.23) (see [29] Introduction Theorem 4.5 or [38] Chapter V §5).

Lemma 5.3 Let $g \in \mathrm{G}, g \notin \mathrm{K}, \lambda>2,0<\eta<\lambda, b \geq 0, B>1$, and let $f$ be a left $\mathrm{K}$-invariant positive continuous function on $\mathrm{G}$. Assume that

$$
A_{g} f \leq \tau_{\lambda}(g) f+b \tau_{\eta}
$$

and that

$$
f(y h) \leq B f(h) \text { if } h, y \in \mathrm{G} \text { and }\|y\| \leq\|g\| .
$$


Then for all $h \in G$

$$
\left(A_{h} f\right)(1)=\int_{\mathrm{K}} f(h k) \mathrm{d} \sigma(k) \leq s \tau_{\lambda}(h),
$$

where

$$
s=B\left(f(1)+\frac{b}{\tau_{\lambda}(g)-\tau_{\eta}(g)}\right) .
$$

Proof We define

$$
f_{\mathrm{K}}(h) \stackrel{\text { def }}{=} \int_{\mathrm{K}} f(h k) \mathrm{d} \sigma(k), \quad h \in \mathrm{G} .
$$

Since $A_{g}$ commutes with right translations, and $\tau_{\eta}$ is right $\mathrm{K}$-invariant, it follows from (5.25) that $A_{g} f_{\mathrm{K}} \leq \tau_{\lambda}(g) f_{\mathrm{K}}+b \tau_{\eta}$. If $h$ and $y$ are as in (5.26), then $f(y h k) \leq B f(h k)$ for every $k \in \mathrm{K}$ and therefore $f_{\mathrm{K}}(y h) \leq B f_{\mathrm{K}}(h)$. On the other hand, it is clear that

$$
f_{\mathrm{K}}(h)=\left(A_{h} f_{\mathrm{K}}\right)(1)=\left(A_{h} f\right)(1) .
$$

Thus we can replace $f$ by $f_{\mathrm{K}}$ and assume that $f$ is bi-K-invariant. Then we have to prove that $f \leq s \tau_{\lambda}$. Assume the contrary, then $f(h)>s^{\prime} \tau_{\lambda}(h)$ for some $h \in G$ and $s^{\prime}>s$. In view of (5.16) and (5.27), $s^{\prime}>s \geq B f(1)$. From this, (5.20) and (5.26) we get that $\|h\|>\|g\|$ and

$$
f(y h)>\frac{s^{\prime}}{B} \tau_{\lambda}(y h) \quad \text { if } \quad\|y\| \leq\|g\| \text { and }\|y h\| \leq\|h\| .
$$

Using the Cartan decomposition, we see that any $x \in \mathrm{G}$ with $\frac{\|h\|}{\|g\|} \leq\|x\| \leq\|h\|$ can be written as $x=k_{1} y h k_{2}$, where $k_{1}, k_{2} \in \mathrm{K},\|y\| \leq\|g\|$ and $\|y h\| \leq\|h\|$. But the functions $f$ and $\tau_{\lambda}$ are bi-K-invariant. Therefore it follows from (5.28) that

$$
f(x)>\frac{s^{\prime}}{B} \tau_{\lambda}(x) \text { if } \frac{\|h\|}{\|g\|} \leq\|x\| \leq\|h\| .
$$

Let

$$
\begin{aligned}
a_{1} & \stackrel{\text { def }}{=} \frac{s^{\prime}}{B}>f(1)+\frac{b}{\tau_{\lambda}(g)-\tau_{\eta}(g)}, a_{2} \stackrel{\text { def }}{=} \frac{b}{\tau_{\lambda}(g)-\tau_{\eta}(g)}, \text { and } \\
\omega & \stackrel{\text { def }}{=} f-a_{1} \tau_{\lambda}+a_{2} \tau_{\eta} .
\end{aligned}
$$


In view of (5.10) and (5.25), we see that

$$
\begin{aligned}
A_{g} \omega-\tau_{\lambda}(g) \omega & =A_{g}\left(f-a_{1} \tau_{\lambda}+a_{2} \tau_{\eta}\right)-\tau_{\lambda}(g)\left(f-a_{1} \tau_{\lambda}+a_{2} \tau_{\eta}\right) \\
& =\left[A_{g} f-\tau_{\lambda}(g) f\right]-a_{1}\left[A_{g} \tau_{\lambda}-\tau_{\lambda}(g) \tau_{\lambda}\right]+a_{2}\left[A_{g} \tau_{\eta}-\tau_{\lambda}(g) \tau_{\eta}\right] \\
& \leq b \tau_{\eta}+a_{2}\left[\tau_{\eta}(g) \tau_{\eta}-\tau_{\lambda}(g) \tau_{\eta}\right]=0 .
\end{aligned}
$$

Since $\tau_{\lambda}(1)=\tau_{\eta}(1)=1$, we have

$$
\omega(1)=f(1)-a_{1}+a_{2}<0 .
$$

It follows from (5.16) that $a_{2} \geq 0$. Using additionally (5.27) and (5.29), we get that

$$
\begin{aligned}
\omega(x) & =f(x)-a_{1} \tau_{\lambda}(x)+a_{2} \tau_{\eta}(x) \geq f(x)-a_{1} \tau_{\lambda}(x) \\
& >\left(\frac{s^{\prime}}{B}-a_{1}\right) \tau_{\lambda}(x)=0 \quad \text { if } \quad \frac{\|h\|}{\|g\|} \leq\|x\| \leq\|h\| .
\end{aligned}
$$

Let $v \in \mathrm{G}$, satisfying $\|v\| \leq\|h\|$, be a point where the continuous function $\omega$ attains its minimum on the set $\{x \in \mathrm{G}:\|x\| \leq\|h\|\}$. It follows from (5.31) and (5.32) that

$$
\omega(v)<0 \quad \text { and } \quad\|v\| \leq \frac{\|h\|}{\|g\|} .
$$

Because of $\tau_{\lambda}(g)>1$ and $\|g k v\| \leq\|g\|\|v\|$ for all $k \in \mathrm{K}$ we conclude

$$
\left(A_{g} \omega\right)(v)=\int_{\mathrm{K}} \omega(g k v) \mathrm{d} \sigma(k) \geq \omega(v)>\tau_{\lambda}(g) \omega(v) .
$$

Thus, we get a contradiction with (5.30).

As a special case $(\eta=2$ and $b=0)$ of Lemma 5.3, we have the following

Corollary 5.4 Let $g \in \mathrm{G}, g \notin \mathrm{K}, \lambda>2, B>1$, and let $f$ be a left $\mathrm{K}$ invariant positive continuous function on $\mathrm{G}$ satisfying the inequality (5.26). Assume that

$$
A_{g} f \leq \tau_{\lambda}(g) f .
$$

Then for all $h \in G$

$$
\left(A_{h} f\right)(1)=\int_{\mathrm{K}} f(h k) \mathrm{d} \sigma(k) \leq B f(1) \tau_{\lambda}(h) .
$$


Lemma 5.5 Let $g \in \mathrm{G}, g \notin \mathrm{K}, 2<\lambda<\mu, B>1, M>1, n \in \mathbb{N}^{+}$and let $f_{i}, 0 \leq i \leq n$, be left $\mathrm{K}$-invariant positive continuous functions on $\mathrm{G}$. We denote $\min \{i, n-i\}$ by $\bar{i}$ and $\sum_{0 \leq i \leq n} f_{i}$ by $f$. Assume that

$$
\begin{aligned}
f_{i}(y h) & \leq B f_{i}(h) \quad \text { if } 0 \leq i \leq n, \quad h, y \in \mathrm{G} \quad \text { and }\|y\| \leq\|g\|, \\
A_{g} f_{i} & \leq \tau_{\lambda}(g) f_{i}+M \max _{0<j \leq \bar{i}} \sqrt{f_{i-j} f_{i+j}}, \quad 0 \leq i \leq n,
\end{aligned}
$$

so in particular $A_{g} f_{0} \leq \tau_{\lambda}(g) f_{0}$ and $A_{g} f_{n} \leq \tau_{\lambda}(g) f_{n}$. Then there is a constant $C=C(g, \lambda, \mu, B, M, n)$ such that for all $h \in \mathrm{G}$,

$$
\left(A_{h} f\right)(1)=\int_{\mathrm{K}} f(h k) \mathrm{d} \sigma(k) \leq C f(1) \tau_{\mu}(h) .
$$

Proof For any $0<\varepsilon \leq 1$ and $0 \leq i \leq n$ we define

$$
f_{i, \varepsilon}=\varepsilon^{q(i)} f_{i} \quad \text { where } q(i) \stackrel{\text { def }}{=} i(n-i) .
$$

Using the inequality (5.33) for all $i, 0 \leq i \leq n$, we see that

$$
\begin{aligned}
A_{g} f_{i, \varepsilon}=\varepsilon^{q(i)} A_{g} f_{i} & \leq \varepsilon^{q(i)} \tau_{\lambda}(g) f_{i}+\varepsilon^{q(i)} M \max _{0<j \leq \bar{i}} \sqrt{\varepsilon^{-q(i-j)} f_{i-j, \varepsilon} \mathcal{E}^{-q(i+j)} f_{i+j, \varepsilon}} \\
& =\tau_{\lambda}(g) f_{i, \varepsilon}+M \max _{0<j \leq \bar{i}} \varepsilon^{q(i)-\frac{1}{2}[q(i-j)+q(i+j)]} \sqrt{f_{i-j, \varepsilon} f_{i+j, \varepsilon}} .
\end{aligned}
$$

Direct computation shows that

$$
q(i)-\frac{1}{2}[q(i-j)+q(i+j)]=j^{2} .
$$

Hence for all $i, 0 \leq i \leq n$,

$$
A_{g} f_{i, \varepsilon} \leq \tau_{\lambda}(g) f_{i, \varepsilon}+\varepsilon M \max _{0<j \leq \bar{i}} \sqrt{f_{i-j, \varepsilon} f_{i+j, \varepsilon}} .
$$

Let $f_{\varepsilon}:=\sum_{0 \leq i \leq n} f_{i, \varepsilon}$. Summing (5.35) over all $i, 0 \leq i \leq n$, and using the inequalities $f_{\varepsilon}>\sqrt{f_{i-j, \varepsilon} f_{i+j, \varepsilon}}$, which are satisfied for any $1 \leq i \leq n-1$, $0<j \leq \bar{i}$, we get

$$
A_{g} f_{\varepsilon}=\sum_{0 \leq i \leq n} A_{g} f_{i, \varepsilon} \leq \tau_{\lambda}(g) f_{\varepsilon}+\varepsilon M(n-1) f_{\varepsilon}=\left(\tau_{\lambda}(g)+\varepsilon M(n-1)\right) f_{\varepsilon} .
$$

Write

$$
\varepsilon_{0}=\min \left\{1, \frac{\tau_{\mu}(g)-\tau_{\lambda}(g)}{M(n-1)}\right\}
$$


in order to get from $(5.36)$ that

$$
A_{g} f_{\varepsilon_{0}} \leq \tau_{\mu}(g) f_{\varepsilon_{0}}
$$

Since $f_{\varepsilon}$ also satisfies (5.26), we can apply Corollary 5.4 to $f_{\varepsilon_{0}}$ and get that

$$
\left(A_{h} f\right)(1)<\varepsilon_{0}^{-n^{2}}\left(A_{h} f_{\varepsilon_{0}}\right)(1) \leq \varepsilon_{0}^{-n^{2}} f_{\varepsilon_{0}}(1) \tau_{\mu}(h) \leq \varepsilon_{0}^{-n^{2}} B f(1) \tau_{\mu}(h)
$$

for all $h \in \mathrm{G}$. Hence (5.34) is true with $C=\varepsilon_{0}^{-n^{2}} B$.

Proposition 5.6 Let $g \in \mathrm{G}, g \notin \mathrm{K}, d \in \mathbb{N}^{+}, B>1, M>1$. For every $0 \leq i \leq 2 d$, let $\lambda_{i} \geq 2$ and let $f_{i}$ be a left $\mathrm{K}$-invariant positive continuous function on $\mathrm{G}$. We denote $\min \{i, 2 d-i\}$ by $\bar{i}$ and $\sum_{0 \leq i \leq 2 d} f_{i}$ by $f$. Assume that

$$
\begin{aligned}
\lambda_{d} & >\lambda_{i} \text { for any } i \neq d . \\
f_{i}(y h) & \leq B f_{i}(h) \text { if } 0 \leq i \leq 2 d, h, y \in \mathrm{G} \text { and }\|y\| \leq\|g\|, \\
A_{g} f_{i} & \leq \tau_{\lambda_{i}}(g) f_{i}+M \max _{0<j \leq \bar{i}} \sqrt{f_{i-j} f_{i+j}}, \quad 0 \leq i \leq 2 d,
\end{aligned}
$$

in particular,

$$
A_{g} f_{0} \leq \tau_{\lambda_{0}}(g) f_{0} \text { and } A_{g} f_{2 d} \leq \tau_{\lambda_{2 d}}(g) f_{2 d} .
$$

Then, using the notation $\ll$ (which until the end of the proof of this proposition means that the left hand side is bounded from above by the right-hand side multiplied by a constant which depends on $g, \lambda_{0}, \ldots, \lambda_{2 d}, B$ and $M$, and does not depend on $f_{0}, \ldots, f_{2 d}$ ), we have that

(a) For all $h \in \mathrm{G}$ and $0 \leq i \leq 2 d, i \neq d$,

$$
\left(A_{h} f_{i}\right)(1)=\int f_{i}(h k) \mathrm{d} \sigma(k) \ll f(1) \tau_{\eta}(h),
$$

where

$$
\eta=\lambda_{d}-3^{-(d+1)}\left(\lambda_{d}-\eta^{\prime}\right)<\lambda_{d}, \quad \eta^{\prime}=\max \left\{\lambda_{i}: 0 \leq i \leq 2 d, i \neq d\right\} .
$$

(b) For all $h \in \mathrm{G}$

$$
\left(A_{h} f_{d}\right)(1)=\int_{\mathrm{K}} f_{d}(h k) \mathrm{d} \sigma(k) \ll f(1) \tau_{\lambda_{d}}(h) .
$$


(c) For all $h \in \mathrm{G}$

$$
\left(A_{h} f\right)(1)=\int_{\mathrm{K}} f(h k) \mathrm{d} \sigma(k) \ll f(1)\|h\|^{\lambda_{d}-2} .
$$

Proof (a) Let

$$
f_{i, \mathrm{~K}}(h) \stackrel{\text { def }}{=} \int_{\mathrm{K}} f_{i}(h k) \mathrm{d} \sigma(k), \quad h \in \mathrm{G} .
$$

The Cauchy-Schwarz inequality implies

$$
\begin{aligned}
\int_{\mathrm{K}} \sqrt{f_{i-j}(h k) f_{i+j}(h k)} \mathrm{d} \sigma(k) & \leq \sqrt{\int_{\mathrm{K}} f_{i-j}(h k) \mathrm{d} \sigma(k)} \sqrt{\int_{\mathrm{K}} f_{i+j}(h k) \mathrm{d} \sigma(k)} \\
& =\sqrt{f_{i-j, \mathrm{~K}}(h) f_{i+j, \mathrm{~K}}(h)} .
\end{aligned}
$$

Hence

$$
\begin{aligned}
\int_{\mathrm{K}} \max _{0<j \leq \bar{i}} \sqrt{f_{i-j}(h k) f_{i+j}(h k)} \mathrm{d} \sigma(k) & \leq \sum_{0<j \leq \bar{i}} \int_{\mathrm{K}} \sqrt{f_{i-j}(h k) f_{i+j}(h k)} \mathrm{d} \sigma(k) \\
& \leq \sum_{0<j \leq \bar{i}} \sqrt{f_{i-j, \mathrm{~K}}(h) f_{i+j, \mathrm{~K}}(h)} \\
& \leq d \max _{0<j \leq \bar{i}} \sqrt{f_{i-j, \mathrm{~K}}(h) f_{i+j, \mathrm{~K}}(h)} .
\end{aligned}
$$

On the other hand, we have

$$
\left(A_{g} f_{i, \mathrm{~K}}\right)(h)=\int_{\mathrm{K}}\left(A_{g} f_{i}\right)(h k) \mathrm{d} \sigma(k)
$$

and according to (5.38)

$$
\left(A_{g} f_{i}\right)(h k) \leq \tau_{\lambda_{i}}(g) f_{i}(h k)+M \max _{0<j \leq i} \sqrt{f_{i-j}(h k) f_{i+j}(h k)} .
$$

Therefore

$$
A_{g} f_{i, \mathrm{~K}} \leq \tau_{\lambda_{i}}(g) f_{i, \mathrm{~K}}+d M \max _{0<j \leq \bar{i}} \sqrt{f_{i-j, \mathrm{~K}} f_{i+j, \mathrm{~K}}} .
$$

But $f_{\mathrm{K}}(1)=f(1)$,

$$
f_{i, \mathrm{~K}}(h)=\left(A_{h} f_{i, \mathrm{~K}}\right)(1)=\left(A_{h} f_{i}\right)(1)
$$


and, as easily follows from (5.37), we have

$$
f_{i, \mathrm{~K}}(y h) \leq B f_{i, \mathrm{~K}}(h)
$$

if $h, y \in \mathrm{G}$, and $\|y\| \leq\|g\|$. Thus, replacing $f_{i}$ by $f_{i, \mathrm{~K}}$ and $M$ by $d M$, we can assume that the functions $f_{i}$ are bi-K-invariant. Then we have to prove that

$$
f_{i} \ll f(1) \tau_{\eta} \text { for all } 0 \leq i \leq 2 d, i \neq d .
$$

Let $\eta^{\prime}=\max \left\{\lambda_{i}: 0 \leq i \leq 2 d, i \neq d\right\}$, as in (5.39). We define $\mu_{i}$, $0 \leq i \leq 2 d$, by

$$
\begin{aligned}
\mu_{d} & =\lambda_{d}+3^{-(d+1)}\left(\lambda_{d}-\eta^{\prime}\right) \text { and } \\
\mu_{i} & =\mu_{d}-3^{-\bar{i}}\left(\lambda_{d}-\eta^{\prime}\right), 0 \leq i \leq 2 d, i \neq d .
\end{aligned}
$$

Since (5.16) implies $\tau_{\lambda_{i}}(g) \leq \tau_{\mu_{d}}(g)$, it follows from (5.16) and Lemma 5.5 that

$$
f_{i} \ll f(1) \tau_{\mu_{d}}, \quad 0 \leq i \leq 2 d .
$$

One can easily check that $\eta>\mu_{i}>\lambda_{i} \geq 2$ and therefore $\tau_{\eta} \geq \tau_{\mu_{i}}$ for all $0 \leq i \leq 2 d, i \neq d$. Thus, to prove (5.40), it is enough to show that

$$
f_{i} \ll f(1) \tau_{\mu_{i}} \text { for all } 0 \leq i \leq 2 d, i \neq d .
$$

We will prove (5.44) for $i \leq d-1$ by using induction in $i$; the proof in the case $i \geq d+1$ is similar. For $i=0$ we have $\tau_{\mu_{0}}(g)>\tau_{\lambda_{0}}(g)$ because of (5.16) and thus it is enough to use Corollary 5.4. Let $1 \leq m \leq d-1$ and assume that (5.44) is proved for all $i<m$. Using (5.43) for all $0<j \leq m$ we find that

$$
\sqrt{f_{m-j} f_{m+j}} \ll f(1) \sqrt{\tau_{\mu_{m-j}} \tau_{\mu_{d}}} \leq f(1) \sqrt{\tau_{\mu_{m-1}} \tau_{\mu_{d}}} \ll f(1) \tau_{\left(\mu_{m-1}+\mu_{d}\right) / 2} .
$$

Note that the second inequality in (5.45) follows from (5.16) and (5.42), and the third one follows from (5.17) and (5.22).

Combining (5.38) and (5.40) we get

$$
A_{g} f_{m} \leq \tau_{\lambda_{m}}(g) f_{m}+C f(1) \tau_{\left(\mu_{m-1}+\mu_{d}\right) / 2},
$$

where $C \ll 1$. On the other hand, we have $\lambda_{m}<\mu_{m}$ and $\left(\mu_{m-1}+\mu_{d}\right) / 2<$ $\mu_{m}$ by (5.41) and (5.42). Now, to prove that $f_{m} \ll f(1) \tau_{\mu_{m}}$, it remains to apply Lemma 5.3 combined with (5.16). 
(b) As in the proof of (a), we can assume that the functions $f_{i}$ are bi- $K$ invariant. Then we get from (5.38) and (5.40) that

$$
A_{g} f_{d} \leq \tau_{\lambda_{d}} f_{d}+D f(1) \tau_{\eta}
$$

where $D \ll 1$. Since $\eta<\lambda_{d}$, Lemma 5.3 implies that $f_{d} \ll f(1) \tau_{\lambda_{d}}$ which proves (b).

(c) Follows from (a), (b), (5.16), (5.17) and (5.22).

\subsection{Quasinorms and representations of $\operatorname{SL}(2, \mathbb{R})$}

We say that a continuous function $v \mapsto|v|$ on a real topological vector space $V$ is a quasinorm if it satisfies the following properties

(i) $|v| \geq 0$ and $|v|=0$ if and only if $v=0$,

(ii) $|\lambda v|=|\lambda| \cdot|v|$ for all $\lambda \in \mathbb{R}$ and $v \in V$.

If $V$ is finite dimensional, then any two quasinorms on $V$ are equivalent in the sense that their ratio lies between two positive constants.

Lemma 5.7 Let $\rho$ be a (continuous) representation of $\mathrm{G}=\mathrm{SL}(2, \mathbb{R})$ in a real topological vector space $V$, let $|\cdot|$ be a $\rho(\mathrm{K})$-invariant quasinorm on $V$ and let $v \in V, v \neq 0$, be an eigenvector for $\rho$ corresponding to the character $\chi_{-r}, r \in \mathbb{R}$, that is

$$
\rho\left(\begin{array}{ll}
a & b \\
0 & a^{-1}
\end{array}\right) v=a^{r} v
$$

Then for any $g \in \mathrm{G}$ and $\beta \in \mathbb{R}$

$$
|\rho(g) v|^{-\beta}=\varphi_{\beta r}(g)|v|^{-\beta}
$$

and

$$
\int_{\mathrm{K}} \frac{\mathrm{d} \sigma(k)}{|\rho(g k) v|^{\beta}}=\tau_{\beta r}(g)|v|^{-\beta}
$$

Proof Using the $\mathrm{K}$-invariance of $|\cdot|$ we get that

$$
\begin{aligned}
|\rho(g) v|^{-\beta} & =|\rho(k(g)) \rho(t(g)) v|^{-\beta}=|\rho(t(g)) v|^{-\beta}=\left|\chi_{-r}(t(g)) v\right|^{-\beta}=\chi_{\beta r}(t(g))|v|^{-\beta} \\
& =\varphi_{\beta r}(g)|v|^{-\beta} .
\end{aligned}
$$

The equality (5.47) follows from (5.46) and from the definition of $\tau_{\beta r}(g)$. 
Let $\|z\|$ denote the norm of $z \in \mathbb{C}^{2}$ corresponding to the standard Hermitian inner product on $\mathbb{C}^{2}$, that is

$$
\|z\|^{2}=\|x\|^{2}+\|y\|^{2} \quad \text { where } z=x+i y, x, y \in \mathbb{R}^{2} .
$$

Lemma 5.8 For any $z \in \mathbb{C}^{2}, z \neq 0, g \in G$ and $\beta>0$, we have

$$
F(z)=F_{g, \beta}(z) \stackrel{\text { def }}{=}\|z\|^{\beta} \int_{\mathrm{K}} \frac{\mathrm{d} \sigma(k)}{\|g k z\|^{\beta}} \leq \tau_{\beta}(g) .
$$

Proof Since the measure $\sigma$ on $\mathrm{K}$ is translation invariant, we have

$$
F(k z)=F(z) \text { for any } k \in \mathrm{K} \text {. }
$$

Also for all $\lambda \in \mathbb{C}, \lambda \neq 0$, and $z \in \mathbb{C}^{2}, z \neq 0$,

$$
F(\lambda z)=F(z),
$$

because $\|\lambda v\|=|\lambda| \cdot\|v\|, v \in \mathbb{C}^{2}$, and because $\mathrm{G}=\operatorname{SL}(2, \mathbb{R})$ acts $\mathbb{C}$-linearly on $\mathbb{C}^{2}$. Any non-zero vector $x \in \mathbb{R}^{2}$ can be represented as $x=\lambda k e_{1}$ with $\lambda \in \mathbb{R}, k \in \mathrm{K}, e_{1}=(1,0)$. Then, using (5.12) from Sect. 5.2, we get from (5.49) and (5.50) that

$$
F(x)=F\left(e_{1}\right)=\tau_{\beta}(g) \text { for all } x \in \mathbb{R}^{2}, x \neq 0 .
$$

Let now $z=x+i y, x, y \in \mathbb{R}^{2}, z \neq 0$. We write $e^{i \theta} z=x_{\theta}+i y_{\theta}, x_{\theta}, y_{\theta} \in$ $\mathbb{R}^{2}$. Then $\frac{\left\|x_{\theta}\right\|}{\left\|y_{\theta}\right\|}$ is a continuous function of $\theta$ with values in $\mathbb{R}_{\geq 0} \cup\{\infty\}$. But $e^{i \pi / 2} z=i z=-y+i x$ and therefore $\frac{\left\|x_{\pi / 2}\right\|}{\left\|y_{\pi / 2}\right\|}=\left(\frac{\left\|x_{0}\right\|}{\left\|y_{0}\right\|}\right)^{-1}$. Hence there exists $\theta$ such that $\left\|x_{\theta}\right\|=\left\|y_{\theta}\right\|$. Replacing then $z$ by $e^{i \theta} z$ and using (5.50) we can assume that $\left\|x_{\theta}\right\|=\left\|y_{\theta}\right\|$. Now using the convexity of the function $t \rightarrow t^{-\beta / 2}$, $t>0$, and the identity (5.51) we get that

$$
\begin{aligned}
\int_{\mathrm{K}} \frac{\mathrm{d} \sigma(k)}{\|g k z\|^{\beta}} & =\int_{\mathrm{K}} \frac{\mathrm{d} \sigma(k)}{\left(\|g k x\|^{2}+\|g k y\|^{2}\right)^{\beta / 2}} \\
& \leq \frac{2^{-\beta / 2}}{2}\left[\int_{K} \frac{\mathrm{d} \sigma(k)}{\|g k x\|^{\beta}}+\int_{\mathrm{K}} \frac{\mathrm{d} \sigma(k)}{\|g k y\|^{\beta}}\right]=\frac{2^{-\beta / 2}}{2}\left[\frac{\tau_{\beta}(g)}{\|x\|^{\beta}}+\frac{\tau_{\beta}(g)}{\|y\|^{\beta}}\right] \\
& =2^{-\beta / 2} \tau_{\beta}(g) \frac{1}{\|x\|^{\beta}}=2^{-\beta / 2} \tau_{\beta}(g) \cdot \frac{1}{\|z\|^{\beta} \cdot 2^{-\beta / 2}}=\frac{\tau_{\beta}(g)}{\|z\|^{\beta}} .
\end{aligned}
$$

Clearly the last inequality (5.52) implies (5.48).

Let us recall some basic facts of the finite-dimensional representation theory of $\mathrm{G}=\mathrm{SL}(2, \mathbb{R})$. Let $W$ be a finite-dimensional complex vector space, 
there is a correspondence between complex-linear representations of $\mathfrak{s l}(2, \mathbb{C})$ on $W$ and representations of $\mathrm{G}$ on $W$, under which invariant subspaces and equivalences are preserved (see [32] Proposition 2.1). It is well-known that any finite-dimensional representation of $\mathfrak{s l}(2, \mathbb{C})$ is fully reducible, that is, it can be decomposed into the direct sum of irreducible representations (see [33] Corollary 1.70). Moreover, for each $m \geq 1$ there exists up to equivalence a unique irreducible complex-linear representation of $\mathfrak{s l}(2, \mathbb{C})$ on a complex vector space of dimension $m$ (see [33] Corollary 1.63). Hence, any finitedimensional representation of $\mathrm{G}$ is fully reducible and any two irreducible finite-dimensional representations of the same degree must be isomorphic. Let $\mathcal{P}_{m}$ denote the $(m+1)$-dimensional complex vector space of complex polynomials in two variables homogeneous of degree $m$, and let $\psi_{m}$ denote the regular representation of $\mathrm{G}=\mathrm{SL}(2, \mathbb{R})$ on $\mathcal{P}_{m}$ defined by $\left(\psi_{m}(g) P\right)(z)=P\left(g^{-1} z\right)$, for $g \in \mathrm{G}, z \in \mathbb{C}^{2}$ and $P \in \mathcal{P}_{m}$. It is well-known that the representation $\psi_{m}$ is irreducible for any $m$ (see [34] Example 2.7.11) and hence it is, up to isomorphism, the unique irreducible finite-dimensional representation of $\mathrm{G}$ of degree $m$. We define

$$
I(\rho)=\left\{m \in \mathbb{N}^{+}: \psi_{m} \text { is isomorphic to a subrepresentation of } \rho\right\} .
$$

Proposition 5.9 Let $\rho$ be a representation of $\mathrm{G}=\mathrm{SL}(2, \mathbb{R})$ on a finitedimensional space $W$. Then there exists a $\rho(\mathrm{K})$-invariant quasinorm $|\cdot|=$ $|\cdot|_{\rho}$ on $W$ such that for any $w \in W, w \neq 0, g \in \mathrm{G}$ and $\beta>0$,

$$
\int_{\mathrm{K}} \frac{\mathrm{d} \sigma(k)}{|\rho(g k) w|^{\beta}} \leq \max _{m \in I(\rho)}\left\{\tau_{\beta m}(g)\right\} \frac{1}{|w|^{\beta}}
$$

Proof Let $W=\bigoplus_{i=1}^{n} W_{i}$ be the decomposition of $W$ into the direct sum of $\rho(\mathrm{G})$-irreducible subspaces, and let $\pi_{i}: W \rightarrow W_{i}$ denote the natural projection. Suppose that we constructed for each $i$ a K-invariant quasinorm $|\cdot|_{i}=|\cdot|_{\rho_{i}}$ on $W_{i}$ such that for any $w \in W_{i}, w \neq 0, g \in \mathrm{G}$, and $\beta>0$,

$$
\int_{\mathrm{K}} \frac{\mathrm{d} \sigma(k)}{\left|\rho_{i}(g k) w\right|_{i}^{\beta}} \leq \tau_{\beta m(i)}(g) \frac{1}{|w|_{i}^{\beta}},
$$

where $\rho_{i}$ denotes the restriction of $\rho$ to $W_{i}$ and $m(i) \in I(\rho)$ is defined by the condition that $\psi_{m(i)}$ is isomorphic to $\rho_{i}$. Then we define $|w|=|w|_{\rho}$ by

$$
|w|=\max _{1 \leq i \leq n}\left|\pi_{i}(w)\right|_{i}, \quad w \in W
$$


Clearly $|\cdot|_{\rho}$ is a $\mathrm{K}$-invariant quasinorm. Let us fix now $w \in W, w \neq 0$. Then

$$
\begin{aligned}
\int_{\mathrm{K}} \frac{\mathrm{d} \sigma(k)}{|\rho(g k) w|^{\beta}} & \leq \min _{1 \leq i \leq n} \int_{\mathrm{K}} \frac{\mathrm{d} \sigma(k)}{\left|\pi_{i}(\rho(g k) w)\right|_{i}^{\beta}}=\min _{1 \leq i \leq n} \int_{\mathrm{K}} \frac{\mathrm{d} \sigma(k)}{\left|\rho_{i}(g k) \pi_{i}(w)\right|_{i}^{\beta}} \\
& \leq \min _{1 \leq i \leq n} \tau_{\beta m(i)}(g) \frac{1}{\left|\pi_{i}(w)\right|_{i}^{\beta}} \leq \max _{m \in I(\rho)}\left\{\tau_{\beta m}(g)\right\} \frac{1}{|w|^{\beta}} .
\end{aligned}
$$

Thus, it is enough to prove the proposition for representations $\psi_{m}$. For this, let $P \in \mathcal{P}_{m}, P \neq 0$. We consider $P$ as a polynomial on $\mathbb{C}^{2}$ and decompose $P$, using the fundamental theorem of algebra, into the product of $m$ linear forms

$$
P=\ell_{1} \cdot \ldots \cdot \ell_{m}, \quad \text { where } \ell_{i}\left(z_{1}, z_{2}\right)=a_{i} z_{1}+b_{i} z_{2}, \quad a_{i}, b_{i}, z_{1}, z_{2} \in \mathbb{C} .
$$

There is a natural $\mathrm{K}$-invariant norm on the space of linear forms on $\mathbb{C}^{2}$ :

$$
\|\ell\|^{2}=|a|^{2}+|b|^{2}, \quad \ell\left(z_{1}, z_{2}\right)=a z_{1}+b z_{2} .
$$

Now we define a quasinorm on $\mathcal{P}_{m}$ by the equation

$$
|P|=\left\|\ell_{1}\right\| \cdot \ldots \cdot\left\|\ell_{m}\right\|
$$

This definition is correct because the factorization (5.55) is unique up to the order of factors and the multiplication of $\ell_{i}, 1 \leq i \leq n$, by constants. We denote by $\tilde{\psi}_{1}$ the extension of $\psi_{1}$ to the space of linear forms on $G$. It is isomorphic to the standard representation of $\mathrm{G}$ on $\mathbb{C}^{2}$. Then using Lemma 5.8 and the generalized Hölder inequality, we get that

$$
\begin{aligned}
\int_{\mathrm{K}} \frac{\mathrm{d} \sigma(k)}{\left|\psi_{m}(g k) P\right|^{\beta}}=\int_{\mathrm{K}} \frac{\mathrm{d} \sigma(k)}{\prod_{i=1}^{m}\left\|\tilde{\psi}_{1}(g k) \ell_{i}\right\|^{\beta}} & \leq \prod_{i=1}^{m}\left(\int_{\mathrm{K}} \frac{\mathrm{d} \sigma(k)}{\left\|\tilde{\psi}_{1}(g k) \ell_{i}\right\|^{\beta m}}\right)^{1 / m} \\
& \leq \prod_{i=1}^{m}\left(\frac{\tau_{\beta m}(g)}{\left\|\ell_{i}\right\|^{\beta m}}\right)^{1 / m}=\frac{\tau_{\beta m}(g)}{|P|^{\beta}} .
\end{aligned}
$$

Since $I\left(\psi_{m}\right)=\{m\}$, (5.56) implies (5.53) for $\rho=\psi_{m}$.

We recall from Sect. 5.2, see (5.15) and (5.16), that $\tau_{\mu}(g)<1$ and $\tau_{\eta}(g)<$ $\tau_{\lambda}(g)$ for any $g \notin \mathrm{K}, 0<\mu<2, \lambda \geq 2$ and $0<\eta<\lambda$. Using this, we deduce from the previous Proposition 5.9 the following corollary.

Corollary 5.10 Let $\rho$ be a representation of $\mathrm{G}=\mathrm{SL}(2, \mathbb{R})$ in a finite dimensional space $W$, and let $m$ be the largest number in $I(\rho)$. Then there exists a $\rho(\mathrm{K})$-invariant quasinorm $|\cdot|=|\cdot|_{\rho}$ on $W$ such that 
(i) if $\beta>0$ and $\beta m \geq 2$ then for any $w \in W, w \neq 0$, and $g \in \mathrm{G}$

$$
\int_{\mathrm{K}} \frac{\mathrm{d} \sigma(k)}{|\rho(g k) w|^{\beta}} \leq \tau_{\beta m}(g) \frac{1}{|w|^{\beta}},
$$

(ii) if $\beta>0$ and $\beta m<2$ then for any $w \in W, w \neq 0$, and $g \in \mathrm{G}, g \notin \mathrm{K}$,

$$
\int_{\mathrm{K}} \frac{\mathrm{d} \sigma(k)}{|\rho(g k) w|^{\beta}}<\frac{1}{|w|^{\beta}} .
$$

\subsection{Functions $\alpha_{i}$ on the space of lattices and estimates for $A_{h} \alpha_{i}$}

Let $\rho$ be a representation of $\mathrm{G}=\mathrm{SL}(2, \mathbb{R})$ on $\mathbb{R}^{n}$ and for each $1 \leq i \leq n$ let $|\cdot|_{i}$ be a $\left(\wedge^{i} \rho\right)(\mathrm{K})$-invariant quasinorm on the exterior product $\wedge^{i} \mathbb{R}^{n}$. Throughout this section the underlying quasinorms in the definition of the lattice functions $\alpha_{i}$ and $\alpha$ are taken to be with respect to this particular choice of quasinorms (see (4.2) and (4.3)). For every compact subset $A \subset \mathrm{G}$ note that

$$
\begin{aligned}
& \sup \left\{\frac{\left|\left(\wedge^{i} \rho\right)(h) v\right|_{i}}{|v|_{i}}: h \in A, v \in \wedge^{i} \mathbb{R}^{n}, v \neq 0\right\} \\
& =\sup \left\{\left|\left(\wedge^{i} \rho\right)(h) v\right|_{i}: h \in A, v \in \wedge^{i} \mathbb{R}^{n},|v|_{i}=1\right\}
\end{aligned}
$$

is finite for every $i, 1 \leq i \leq n$. Hence, if we fix $g \in \mathrm{G}, g \notin \mathrm{K}$, then there exists some $B>1$ such that for any $i, 1 \leq i \leq n$, and $v \in \wedge^{i} \mathbb{R}^{n}, v \neq 0$,

$$
B^{-1}<\frac{\left|\left(\wedge^{i} \rho\right)(y) v\right|_{i}}{|v|_{i}}<B \text { if } y \in \mathrm{G} \text { and }\|y\| \leq\|g\|,
$$

where $\|h\|=\left\|h^{-1}\right\|$ denotes the norm of $h \in \mathrm{G}=\mathrm{SL}(2, \mathbb{R})$ with respect to the standard Euclidean norm on $\mathbb{R}^{2}$. Now, let $\Delta$ be a lattice in $\mathbb{R}^{n}$ and $L$ a $\Delta$-rational subspace. For any $h \in \operatorname{SL}(2, \mathbb{R})$ observe that $h L$ is an $h \Delta$-rational subspace and if $v_{1}, \ldots, v_{i}$ is a basis of $\Delta \cap L$ then $h v_{1}, \ldots, h v_{i}$ is a basis of $h \Delta \cap h L$. This observation together with (5.57) implies that

$$
B^{-1}<\frac{d_{y \Delta}(y L)}{d_{\Delta}(L)}<B \text { if } y \in \mathrm{G} \text { and }\|y\| \leq\|g\| .
$$

Hence, for any $i \in\{0, \ldots, n\}$ it follows that

$$
\alpha_{i}(y \Delta)<B \alpha_{i}(\Delta) \text { if } y \in \mathrm{G} \text { and }\|y\| \leq\|g\| .
$$


For any $\beta>0$ and $1 \leq i \leq n$ we define the functions $F_{i, \beta}$ on $\wedge^{i} \mathbb{R}^{n} \backslash\{0\}$ by

$$
F_{i, \beta}(w) \stackrel{\text { def }}{=} \int_{K} \frac{|w|_{i}^{\beta}}{\left|\left(\wedge^{i} \rho\right)(g k) w\right|_{i}^{\beta}} \mathrm{d} \sigma(k), \quad w \in \wedge^{i} \mathbb{R}^{n}, w \neq 0 .
$$

It is clear that the functions $F_{i, \beta}$ are continuous and that $F_{i, \beta}(\lambda w)=F_{i, \beta}(w)$ for any $\lambda \in \mathbb{R}, \lambda \neq 0$. Let $c_{0, \beta}:=1$ and for $1 \leq i \leq n$

$$
c_{i, \beta} \stackrel{\text { def }}{=} \sup \left\{F_{i, \beta}(w): w \in \wedge^{i} \mathbb{R}^{n}, w \neq 0\right\}=\sup \left\{F_{i, \beta}(w): w \in \wedge^{i} \mathbb{R}^{n},|w|_{i}=1\right\} .
$$

We note that $c_{n, \beta}=1$, since the image of any continuous homomorphism $\operatorname{SL}(2, \mathbb{R}) \rightarrow \operatorname{GL}(n, \mathbb{R})$ is contained in $\operatorname{SL}(n, \mathbb{R})$ and thus $\left|\left(\wedge^{n} \rho\right)(g k) w\right|_{n}=$ $\left|\operatorname{det}\left(\wedge^{n} \rho(g k)\right)\right||w|_{n}=|w|_{n}$.

Lemma 5.11 For any $i, 0 \leq i \leq n$,

$$
A_{g} \alpha_{i}^{\beta} \leq c_{i, \beta} \alpha_{i}^{\beta}+C^{\beta} B^{2 \beta} \max _{0<j \leq \bar{i}} \sqrt{\alpha_{i-j}^{\beta} \alpha_{i+j}^{\beta}},
$$

where $\bar{i}=\min \{i, n-i\}$, the constant $C \geq 1$ is from Lemma 4.1 and the operator $A_{g}$ is defined by (5.8) from Sect. 5.2.

Proof Let $\Delta$ be a lattice in $\mathbb{R}^{n}$. We have to prove that

$$
\int_{\mathrm{K}} \alpha_{i}(g k \Delta)^{\beta} \mathrm{d} \sigma(k) \leq c_{i, \beta} \alpha_{i}(\Delta)^{\beta}+C^{\beta} B^{2 \beta} \max _{0<j \leq \bar{i}} \sqrt{\alpha_{i-j}(\Delta)^{\beta} \alpha_{i+j}(\Delta)^{\beta}} .
$$

According to Remark 2.1 there exists a $\Delta$-rational subspace $L$ of dimension $i$ such that

$$
\frac{1}{d_{\Delta}(L)}=\alpha_{i}(\Delta)
$$

Let us denote the set of $\Delta$-rational subspaces $M$ of dimension $i$ with $d_{\Delta}(M)<$ $B^{2} d_{\Delta}(L)$ by $\Psi_{i}$. For a $\Delta$-rational $i$-dimensional subspace $M \notin \Psi_{i}$ we get from (5.58) that

$$
d_{g k \Delta}(g k M)>d_{g k \Delta}(g k L) .
$$

If $\Psi_{i}=\{L\}$, then it follows from this and the definitions of $\alpha_{i}$ and $c_{i, \beta}$ that

$$
\int_{\mathrm{K}} \alpha_{i}(g k \Delta)^{\beta} \mathrm{d} \sigma(k) \leq c_{i, \beta} \alpha_{i}(\Delta)^{\beta}
$$


Assume now that $\Psi_{i} \neq\{L\}$. Let $M \in \Psi_{i}, M \neq L$. Then $\operatorname{dim}(M+L)=i+j$, $0<j \leq \bar{i}$. Now we obtain by (5.58), (5.63) and Lemma 4.1 for any $k \in K$ that

$$
\begin{aligned}
\alpha_{i}(g k \Delta)<B \alpha_{i}(\Delta)=\frac{B}{d_{\Delta}(L)} \leq \frac{B^{2}}{\sqrt{d_{\Delta}(L) d_{\Delta}(M)}} & \leq \frac{C B^{2}}{\sqrt{d_{\Delta}(L \cap M) d_{\Delta}(L+M)}} \\
& \leq C B^{2} \sqrt{\alpha_{i-j}(\Delta) \alpha_{i+j}(\Delta)} .
\end{aligned}
$$

Hence, if $\Psi_{i} \neq\{L\}$,

$$
\int_{\mathrm{K}} \alpha_{i}(g k \Delta)^{\beta} \mathrm{d} \sigma(k) \leq C^{\beta} B^{2 \beta} \max _{0<j \leq i} \sqrt{\alpha_{i-j}(\Delta)^{\beta} \alpha_{i+j}(\Delta)^{\beta}} .
$$

Combining (5.64) and (5.65), we get (5.62).

Theorem 5.12 Let $d \in \mathbb{N}^{+}$and let $\rho_{d}$ be a representation of $\mathrm{G}=\mathrm{SL}(2, \mathbb{R})$ isomorphic to the direct sum of $d$ copies of the standard 2-dimensional representation. Let $\beta$ be a positive number such that $\beta d>2$. Then there is a constant $R$, depending only on $\beta$ and the choice of the $\mathrm{K}$-invariant quasinorms $|\cdot|{ }_{i}$ involved in the definition of $\alpha_{i}$, such that for any $h \in \mathrm{G}$ and any lattice $\Delta$ in $\mathbb{R}^{2 d}$

$$
\left(A_{h} \alpha^{\beta}\right)(\Delta)=\int_{\mathrm{K}} \alpha(h k \Delta)^{\beta} \mathrm{d} \sigma(k) \leq R \alpha(\Delta)^{\beta}\|h\|^{\beta d-2} .
$$

Proof As in Sect. 5.3, we define for a finite dimensional representation $\rho$ of G

$$
I(\rho)=\left\{m \in \mathbb{N}^{+}: \psi_{m} \text { is isomorphic to a subrepresentation of } \rho\right\},
$$

where $\psi_{m}$ denotes the regular representation of $\mathrm{G}$ in the space of complex homogeneous polynomials in two variables homogeneous of degree $m$. Let $m_{i}$ be the largest number in $I\left(\wedge^{i} \rho_{d}\right), 1 \leq i \leq 2 d$. It is well known that

$$
m_{i}=\bar{i} \stackrel{\text { def }}{=} \min \{i, 2 d-i\} .
$$

We fix $g \in \mathrm{G}, g \notin \mathrm{K}$. It follows from (5.66) and from Corollary 5.10 that we can choose quasinorms $|\cdot|_{i}$ on $\wedge^{i} \mathbb{R}^{2 d}$ in such a way that for $w \in \wedge^{i} \mathbb{R}^{2 d}$, $w \neq 0$,

$$
\int_{\mathrm{K}} \frac{|w|_{i}^{\beta}}{\left|\left(\wedge^{i} \rho_{d}\right)(g) w\right|_{i}^{\beta}} \mathrm{d} \sigma(k) \leq \begin{cases}\tau_{\beta \bar{i}}(g) & \text { if } \beta \bar{i} \geq 2 \\ 1 & \text { if } \beta \bar{i}<2 .\end{cases}
$$


Hence

$$
c_{i, \beta} \leq \tau_{\beta \bar{i}}(g) \text { if } \beta \bar{i} \geq 2 \text { and } c_{i, \beta} \leq 1 \text { if } \beta \bar{i}<2 .
$$

where $c_{i, \beta}, 1 \leq i \leq 2 d$, is defined by (5.60) and $c_{0, \beta}=1$. As a remark, we notice that $c_{i, \beta}=\tau_{\beta \bar{i}}(g)$ if $\beta \bar{i} \geq 2$.

According to Lemma 5.11, the functions $\alpha_{i}^{\beta}, 0 \leq i \leq 2 d$, satisfy the following system of inequalities

$$
A_{g} \alpha_{i}^{\beta} \leq c_{i, \beta} \alpha_{i}^{\beta}+C^{\beta} B^{2 \beta} \max _{0<j \leq \bar{i}} \sqrt{\alpha_{i-j}^{\beta} \alpha_{i+j}^{\beta}}
$$

Let

$$
\lambda_{i} \stackrel{\text { def }}{=} \max \{2, \beta \bar{i}\}, \quad 0 \leq i \leq 2 d .
$$

Since $\tau_{2}(g)=1$, see (5.14) in Sect. 5.2, it follows from (5.67)-(5.69) that

$$
A_{g} \alpha_{i}^{\beta} \leq \tau_{\lambda_{i}}(g) \alpha_{i}^{\beta}+C^{\beta} B^{2 \beta} \max _{0<j \leq i} \sqrt{\alpha_{i-j}^{\beta} \alpha_{i+j}^{\beta}}, \quad 0 \leq i \leq 2 d .
$$

Now we fix a lattice $\Delta$ in $\mathbb{R}^{2 d}$ and define functions $f_{i}, 0 \leq i \leq 2 d$, on $\mathrm{G}$ by

$$
f_{i}(h)=\alpha_{i}(h \Delta)^{\beta}, \quad h \in \mathrm{G} .
$$

Then it follows from (5.70) that

$$
A_{g} f_{i} \leq \tau_{\lambda_{i}}(g) f_{i}+C^{\beta} B^{2 \beta} \max _{0<j \leq \bar{i}} \sqrt{f_{i-j} f_{i+j}}, \quad 0 \leq i \leq 2 d .
$$

On the other hand, in view of (5.59),

$$
f_{i}(y h) \leq B^{\beta} f_{i}(h), \quad \text { if } \quad 0 \leq i \leq 2 d, h, y \in \mathrm{G} \quad \text { and } \quad\|y\| \leq\|g\| .
$$

Since $\beta d>2$, we have that $\beta d=\lambda_{d}>\lambda_{i}$ for any $i \neq d$. Now we can apply Proposition 5.6 (c) in order to get that

$$
\begin{aligned}
\left(A_{h} \alpha^{\beta}\right)(\Delta)<\left(A_{h} \sum_{0 \leq i \leq 2 d} \alpha_{i}^{\beta}\right)(\Delta) & =\left(A_{h} \sum_{0 \leq i \leq 2 d} f_{i}\right)(1) \ll\left(\sum_{0 \leq i \leq 2 d} f_{i}(1)\right)\|h\|^{\lambda_{d}-2} \\
& =\left(\sum_{0 \leq i \leq 2 d} \alpha_{i}(\Delta)^{\beta}\right)\|h\|^{\lambda_{d}-2} \leq 2 d \alpha(\Delta)^{\beta}\|h\|^{\beta d-2} .
\end{aligned}
$$

The inequality (5.71) proves the theorem for our specific choice of the quasinorms $|\cdot|_{i}$. Now it remains to notice that any two quasinorms on $\wedge^{i} \mathbb{R}^{n}$ are equivalent. 


\section{Proofs of Theorems 2.2 and 1.9}

In this section we shall prove our main theorem, giving effective estimates on the lattice remainder. But, before doing this, we have to establish mean-value estimates for the $\alpha_{d}$-characeristics of $\Lambda_{t}$ by applying Theorem 5.12 combined with Lemma 5.1.

Corollary 6.1 Let $r \geq q^{1 / 2}, I=\left[t_{0}, t_{0}+1\right]$ with $t_{0} \in \mathbb{R}, 0<\beta \leq 1 / 2$ with $\beta d>2$ and $\widehat{g}_{I}:=\max \left\{\left|\widehat{g}_{w}(t)\right|: t \in I\right\}$. Using the notation (5.2), we have

$$
\int_{I} \alpha_{d}\left(\Lambda_{t}\right)^{1 / 2}\left|\widehat{g}_{w}(t)\right| \mathrm{d} t \ll_{\beta, d} q|\operatorname{det} Q|^{-\beta / 2} \widehat{g}_{I} \gamma_{I, \beta}(r) r^{\frac{d}{2}-2},
$$

where $\gamma_{I, \beta}(r)=1$ if $\beta=1 / 2$. Note that we need at least $d \geq 5$.

Based on our variant of Weyl's inequality (see Lemma 3.3 and Corollary 4.5) the $\alpha$-characteristic enters with a power $1 / 2$ in (6.1). While saving a maximum of the $\alpha$-characeristic, it will enter still with an exponent $0<\beta \leq 1 / 2$ for its average (compare Lemma 5.1). Since the crucial averaging recursion (Theorem 5.12) fails unless $\beta d>2$, the proof essentially needs $d>4$ and thus $d \geq 5$.

Proof In order to apply Lemma 5.1, we cover $I$ by intervals $I_{j}=\left[s_{j}, s_{j+1}\right]$ of length at most $1 / q$, where $s_{j}=t_{0}+j / q$ with $j \in J:=\{0, \ldots,\lceil q\rceil\}$. This implies

$$
\begin{aligned}
\int_{I} \alpha_{d}\left(\Lambda_{t}\right)^{1 / 2}\left|\widehat{g}_{w}(t)\right| \mathrm{d} t & \leq r^{\frac{d}{2}-\beta d} \widehat{g}_{I} \gamma_{I, \beta}(r) \frac{1}{q} \sum_{j \in J} \int_{-\pi}^{\pi} \alpha\left(d_{r_{*}} k_{\theta} \Lambda_{Q, s_{j}}\right)^{\beta} \frac{\mathrm{d} \theta}{2 \pi} \\
& \ll r^{\frac{d}{2}-\beta d} \widehat{g}_{I} \gamma_{I, \beta}(r) \max _{j \in J} \int_{-\pi}^{\pi} \alpha\left(d_{r_{*}} k_{\theta} \Lambda_{Q, s_{j}}\right)^{\beta} \frac{\mathrm{d} \theta}{2 \pi} .
\end{aligned}
$$

Now, we shall apply Theorem 5.12 with $h=d_{r_{*}}, r_{*}=r / q^{1 / 2}$ and the lattices $\Lambda_{Q, s_{j}}=d_{q^{1 / 2}} u_{s_{j}} \Lambda_{Q}$, as defined in (5.3), and obtain

$$
\begin{aligned}
\max _{j \in J} \int_{-\pi}^{\pi} \alpha\left(d_{r_{*}} k_{\theta} \Lambda_{Q, s_{j}}\right)^{\beta} \frac{d \theta}{2 \pi} & \ll \beta, d \max _{j \in J} \alpha\left(\Lambda_{Q, s_{j}}\right)^{\beta}\left\|d_{r_{*}}\right\|^{\beta d-2} \\
& \ll_{d} r^{\beta d-2}\left(q d_{Q}^{\beta}\right),
\end{aligned}
$$

where we have used $\left\|d_{r_{*}}\right\|=r_{*}=r / q^{1 / 2}$ and (4.18) in form of

$$
\alpha\left(\Lambda_{Q, s_{j}}\right) \ll_{d} \alpha_{d}\left(\Lambda_{Q, s_{j}}\right) \ll_{d}|\operatorname{det} Q|^{-1 / 2} q^{d / 2} .
$$

Note that we have applied Corollary 4.5 with $r=q^{1 / 2}$ and $t=s_{j}$ in order to get $\alpha\left(\Lambda_{Q, s_{j}}\right) \asymp_{d} \alpha_{d}\left(\Lambda_{Q, s_{j}}\right)$. Finally, in view of (6.2), this concludes the proof of (6.1). 
In order to bound the lattice point remainder for 'wide shells', that is $b-a>$ $q^{1 / 2}$, we need to extend the averaging result, established in Corollary 6.1, for small values of $t$. To do this, we recall the bound

$$
\left|\widehat{g}_{w}(t)\right| \ll \min \left\{|b-a|,|t|^{-1}\right\} \exp \left\{-|t w|^{1 / 2}\right\}
$$

for the integrand $\widehat{g}_{w}(t)$ in (5.4), provided that $0<w<(b-a) / 4$. Note that it is of size $b-a$ for $|t| \leq 1 /(b-a)$ and changes rapidly if $|b-a|>1$ grows with $r$.

Lemma 6.2 If $r \geq q^{1 / 2}, \beta d>2$ and $0<w<|b-a| / 4$, then

$$
\int_{q_{0}^{-1 / 2} r^{-1}}^{q^{-1 / 2}} \alpha_{d}\left(\Lambda_{t}\right)^{1 / 2}\left|\widehat{g}_{w}(t)\right| \mathrm{d} t \ll_{\beta, d} q^{\beta d+1 / 2}|\operatorname{det} Q|^{-\beta / 2} \gamma_{I, \beta}(r) r^{\frac{d}{2}-2},
$$

where $I=\left[q_{0}^{-1 / 2} r^{-1}, q^{-1 / 2}\right]$.

Proof. Proceeding first as in the proof of Lemma 5.1 and changing variables to $s=t^{-1}$ it is plain to see that

$$
\int_{q_{0}^{-1 / 2} r^{-1}}^{q^{-1 / 2}} \alpha_{d}\left(\Lambda_{t}\right)^{1 / 2}\left|\widehat{g}_{w}(t)\right| \mathrm{d} t \ll_{d} \gamma_{I, \beta}(r) r^{d / 2-\beta d} \int_{q^{1 / 2}}^{r q_{0}^{1 / 2}} \alpha_{d}\left(d_{r} u_{4 s^{-1}} \Lambda_{Q}\right)^{\beta}\left|\widehat{g}_{w}\left(s^{-1}\right)\right| \frac{\mathrm{d} s}{s^{2}} .
$$

Let $N=\left\lceil r\left(q_{0} / q\right)^{1 / 2}\right\rceil$, then the integral on the right-hand side is bounded by $\sum_{j=2}^{N} I_{j}$, where

$$
I_{j} \stackrel{\text { def }}{=} \int_{q^{1 / 2}(j-1)}^{q^{1 / 2} j} \alpha_{d}\left(d_{r} u_{4 s^{-1}} \Lambda_{Q}\right)^{\beta}\left|\widehat{g}_{w}\left(s^{-1}\right)\right| \frac{\mathrm{d} s}{s^{2}} .
$$

For $2 \leq j \leq N$ write $t_{j}=q^{-1 / 2} j^{-1}$, then using that

$$
d_{r} u_{4 s^{-1}}=d_{r} u_{4\left(s^{-1}-t_{j}\right)} u_{4 t_{j}}=d_{4 r j^{-1}} u_{4^{-1} j^{2}\left(s^{-1}-t_{j}\right)} d_{4^{-1} j} u_{4 t_{j}}
$$

together with the change of variables $v=4^{-1} j^{2}\left(s^{-1}-t_{j}\right)$ yields

$$
\begin{aligned}
I_{j} & \leq \frac{4}{j^{2}} \int_{0}^{1} \alpha_{d}\left(d_{4 r j^{-1}} u_{v} d_{4^{-1} j} u_{4 t_{j}} \Lambda_{Q}\right)^{\beta}\left|\widehat{g}_{w}\left(4 v j^{-2}+t_{j}\right)\right| \mathrm{d} v \\
& \ll d \frac{q^{1 / 2}}{j} \int_{0}^{1} \alpha_{d}\left(d_{4 r j^{-1}} u_{v} d_{4^{-1} j} u_{4 t_{j}} \Lambda_{Q}\right)^{\beta} \mathrm{d} v,
\end{aligned}
$$

where the last inequality is a consequence of $\left|\widehat{g}_{w}(t)\right| \ll|t|^{-1}$. Hence, since $4 r j^{-1} \geq 1$ and $q^{1 / 2} j t_{j}=1$, we deduce from Lemma 4.7, Theorem 5.12 and 
(4.20) of Lemma 4.6 that

$$
\begin{aligned}
I_{j} & \ll_{d} \frac{q^{1 / 2}}{j} \int_{\mathrm{K}} \alpha_{d}\left(d_{4 r j^{-1}} k d_{4^{-1} j} u_{4 t_{j}} \Lambda_{Q}\right)^{\beta} \mathrm{d} \sigma(k) \\
& \ll_{d} r^{\beta d-2}|\operatorname{det} Q|^{-\beta / 2} q^{\beta d / 2+1 / 2} j^{1-\beta d} \max \left\{1,\left(4 q^{1 / 2} j^{-1}\right)^{\beta d}\right\} .
\end{aligned}
$$

Summing the last inequality over $2 \leq j \leq N$, we observe that it suffices to show that the following estimate holds

$$
\sum_{j=2}^{N} j^{1-\beta d} \max \left\{1,\left(4 q^{1 / 2} j^{-1}\right)^{\beta d}\right\} \ll_{\beta, d} r^{\beta d-2}|\operatorname{det} Q|^{-\beta / 2} q^{\beta d+1 / 2} .
$$

Indeed, split the previous sum according to whether $j \leq 4 q^{1 / 2}$ or $j>4 q^{1 / 2}$. The sum over $j>4 q^{1 / 2}$ can be bounded by $r^{\beta d-2}|\operatorname{det} Q|^{-\beta / 2} q^{\beta d / 2+1 / 2} \sum_{j=\left\lceil 4 q^{1 / 2}\right\rceil}^{N} j^{1-\beta d} \ll_{\beta, d} r^{\beta d-2}|\operatorname{det} Q|^{-\beta / 2} q^{3 / 2}$, and the sum over $2 \leq j \leq 4 q^{1 / 2}$ by

$$
r^{\beta d-2}|\operatorname{det} Q|^{-\beta / 2} q^{\beta d+1 / 2} \sum_{j=2}^{\left\lfloor 4 q^{1 / 2}\right\rfloor} j^{1-2 \beta d} \ll_{\beta, d} r^{\beta d-2}|\operatorname{det} Q|^{-\beta / 2} q^{\beta d+1 / 2} .
$$

Proof of Theorem 2.2 In view of (3.39), it remains to estimate $I_{\theta}$. By (5.1), with $K_{0}:=\left[q_{0}^{-1 / 2} r^{-1}, 1\right]$ and $K_{j}:=(j, j+1], j \geq 1$, we have

$$
I_{\theta} \ll_{d}|\operatorname{det} Q|^{-\frac{1}{4}}\|\widehat{\zeta}\|_{1}\left(I_{\theta, 0}+\sum_{j=1}^{\infty} I_{\theta, j}\right), \text { where } I_{\theta, j} \stackrel{\text { def }}{=} \int_{K_{j}}\left|\widehat{g}_{w}(t)\right| \alpha_{d}\left(\Lambda_{t}\right)^{\frac{1}{2}} \mathrm{~d} t
$$

For fixed $r \geq q^{1 / 2}$ we may choose

$$
0<w<(b-a) / 4, \quad 1 \geq T_{-} \geq q_{0}^{-1 / 2} r^{-1}, \quad T_{+} \geq 1 \quad \text { and } \frac{d}{2}>\beta d>2 .
$$

For notational simplicity, we write $C_{Q}:=q|\operatorname{det} Q|^{-1 / 4-\beta / 2}$.

Step 1: Estimate of $I_{\theta, 0}$. We consider the case $b-a \leq q$ first. Here we apply Corollary 6.1 to bound the integral over $K_{0}$ combined with $\widehat{g}_{K_{0}} \ll$ $s_{[a, b]_{ \pm w}}(t) \ll b-a$, compare (3.8) and (3.9). Note that we didn't use the restriction $b-a \leq q$ at all. For wide shells, i.e. in the case $b-a>q$, we use Lemma 6.2 for $t \in K_{0}, q_{0}^{-1 / 2} r^{-1} \leq|t| \leq q^{-1 / 2}$ and Corollary 6.1 for the other $t$ in $K_{0}$ together with $\widehat{g}_{\left[q^{-1 / 2}, 1\right]} \ll q^{1 / 2}$. Furthermore, for both cases of $b-a$, split $K_{0}=K_{00} \cup K_{01}$, where $K_{00}:=\left[q_{0}^{-1 / 2} r^{-1}, T_{-}\right]$and $K_{01}:=\left(T_{-}, 1\right]$. 
Then (4.19) of Lemma 4.6 yields

$$
\gamma_{K_{00}, \beta}(r) \ll_{d}\left(|\operatorname{det} Q|^{\frac{1}{2}} T_{-}^{d}\right)^{\frac{1}{2}-\beta}=T_{-}^{\frac{d}{2}-2-\delta}|\operatorname{det} Q|^{\frac{1}{4}-\frac{\beta}{2}},
$$

with the notation (5.2). Using $C_{Q} q^{(2 \beta d-1) / 2}=\bar{C}_{Q}$, we may bound $I_{\theta, 0}$ as

$$
\begin{aligned}
I_{\theta, 0} & \ll{ }_{d} C_{Q}(b-a)_{q}\left(|\operatorname{det} Q|^{\frac{1}{4}-\frac{\beta}{2}} T_{-}^{\frac{d}{2}-2-\delta}+\gamma_{K_{01}, \beta}(r)\right) r^{d-2}, \quad \text { where } \\
(b-a)_{q} & \stackrel{\text { def }}{=}(b-a) I(b-a \leq q)+q^{(2 \beta d-1) / 2} I(b-a>q) .
\end{aligned}
$$

As a side remark, we note that the above splitting of the interval $K_{0}=$ $\left[q_{0}^{-1 / 2} r^{-1}, 1\right]$ is required for our later applications - especially, Corollary 4.11 is only valid for fixed intervals $\left[T_{-}, T_{+}\right]$.

Step 2: Estimate of $I_{\theta, j}$ for $j \geq 1$. Similar as before, applying Corollary 6.1 (with $\beta=1 / 2$ ), while noting that $\gamma_{I, \beta}(r)=1$ if $\beta=1 / 2$, yields

$$
I_{\theta, j} \ll_{d} \widehat{g}_{K_{j}} q|\operatorname{det} Q|^{-1 / 2} r^{d-2} .
$$

We recall the bound (6.3) for $\widehat{g}_{w}$ and the choices of $T_{+}$and $w$ in (6.6) in order to get

$$
\sum_{j=T_{+}}^{\infty} \widehat{g}_{K_{j}} \ll \int_{T_{+}}^{\infty} \frac{\exp \left\{-|s w|^{1 / 2}\right\}}{s} \mathrm{~d} s \ll \frac{1}{\sqrt{T_{+} w}} \exp \left\{-\left|T_{+} w\right|^{1 / 2}\right\}
$$

Thus, we obtain

$$
\sum_{j=T_{+}}^{\infty} I_{\theta, j} \ll_{d} r^{d-2} q|\operatorname{det} Q|^{-1 / 2}\left(T_{+} w\right)^{-1 / 2} \exp \left\{-\left|T_{+} w\right|^{1 / 2}\right\} .
$$

Furthermore, for $b-a>1$ we can use $\left|\widehat{g}_{K_{j}}\right| \ll j^{-1}$ to bound the remaining sum. Whereas for $b-a \leq 1$ we use $\left|\widehat{g}_{K_{j}}\right| \ll b-a$ for $1 \leq j \leq S-1$ and $\left|\widehat{g}_{K_{j}}\right| \ll j^{-1}$ for $S \leq j \leq T_{+}-1$ and minimize the resulting expression in $S$. In both cases this leads to

$$
\sum_{j=1}^{T_{+}-1} \widehat{g}_{K_{j}} \ll 1+\log \left((b-a)^{*} T_{+}\right)
$$

where

$$
(b-a)^{*} \stackrel{\text { def }}{=}(b-a) I(b-a \leq 1)+I(b-a>1)
$$


Hence, using (6.5) combined with (6.8), (6.11) and (6.12) with (6.10), we get

$$
\begin{aligned}
I_{\theta} \ll d\|\widehat{\zeta}\|_{1} r^{d-2} C_{Q} & (b-a)_{q}\left(c_{Q} T_{-}^{\frac{d}{2}-2-\delta}+\gamma_{\left[T_{-}, 1\right], \beta}(r)\right) \\
& \left.+\gamma_{\left(1, T_{+}\right], \beta}(r)\left(1+\log \left((b-a)^{*} T_{+}\right)\right)+c_{Q}^{-1} \frac{\exp \left(-\left(T_{+} w\right)^{1 / 2}\right)}{\left(T_{+} w\right)^{1 / 2}}\right)
\end{aligned}
$$

where $c_{Q}=|\operatorname{det} Q|^{\frac{1}{4}-\frac{\beta}{2}}$. Together with the inequality (3.39) we obtain

$$
\begin{aligned}
\Delta_{r}(\mathrm{v}) & \stackrel{\text { def }}{=}\left|\sum_{m \in \mathbb{Z}^{d}} I_{[a, b]}(Q[m]) \mathrm{v}_{r}(m)-\int_{\mathbb{R}^{d}} I_{[a, b]}(Q[x]) \mathrm{v}_{r}(x) \mathrm{d} x\right| \\
& \ll \beta, d r^{d-2}\left(\|\widehat{\zeta}\|_{1} C_{Q} \rho_{Q, b-a}^{w}(r)+w\|\mathrm{v}\|_{Q}\right)+d_{Q} r^{d / 2}\|\widehat{\zeta}\|_{*, r} \log \left(1+\frac{|b-a|}{q_{0}^{1 / 2} r}\right),
\end{aligned}
$$

where

$$
\begin{array}{r}
\rho_{Q, b-a}^{w}(r) \stackrel{\text { def }}{=} \inf \left\{(b-a)_{q}\left(c_{Q} T_{-}^{\frac{d}{2}-2-\delta}+\gamma_{\left[T_{-}, 1\right], \beta}(r)\right)+\gamma_{\left(1, T_{+}\right], \beta}(r)\left(1+\log \left((b-a)^{*} T_{+}\right)\right)\right. \\
\left.+c_{Q}^{-1}\left(T_{+} w\right)^{-1 / 2} \mathrm{e}^{-\left(T_{+} w\right)^{1 / 2}}: T_{-} \in\left[q_{0}^{-1 / 2} r^{-1}, 1\right], T_{+} \geq 1\right\}
\end{array}
$$

under the condition $0<w<(b-a) / 4$. This completes the proof of Theorem 2.2.

Proof of Theorem 1.9 We have only to apply Theorem 2.2 to the Gaussian weights $\mathrm{v}(x)=\exp \left\{-2 Q_{+}[x]\right\}$ noting that $\zeta(x)=\exp \left\{-Q_{+}[x]\right\}$ satisfies the integrability condition (2.4). This yields

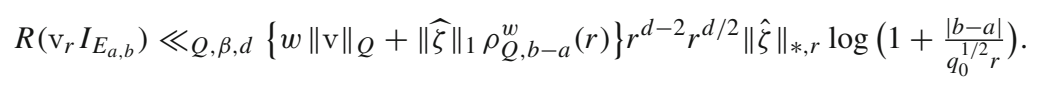

In view of (7.9) and (7.8), we see that $\|\mathrm{v}\|_{Q} \ll d_{Q}$. Here we used that $\varphi_{\mathrm{v}}\left(v, \sqrt{u^{2}-v}\right)=\exp \left\{-2 u^{2}\right\}$, if $Q$ is indefinite; and $\varphi_{\mathrm{v}}(v)=\exp \left\{-2 v^{2}\right\}$ if $Q$ is positive definite. Moreover, a simple calculation shows that $\|\widehat{\zeta}\|_{1} \ll_{d} 1$ and by following the arguments in the proof of (7.31) we get $\|\hat{\zeta}\|_{*, r} \ll_{d}$ $q^{d / 4}\left(\left(q / q_{0}\right)^{d / 2}+d_{Q} q^{d / 2}\right)$ as well.

\section{Lattice point deficiency for admissible regions and applications}

Before we can apply Theorem 2.2, we have to construct smooth bump functions, approximating the indicator function of special parallelepiped regions, and also to control the additional error produced by this smoothing step: In the following Lemma 7.1 we shall bound the volume of $\varepsilon$-boundaries of $r \Omega \cap E_{a, b}$ and in Lemma 7.2 we estimate integrals of the Fourier transform of the region $\Omega$. For wide shells the lattice point counting remainders will reflect the Diophantine properties of $Q$ more directly when using counting regions $\Omega$ which are 'admissible' convex polyhedra. 


\subsection{Smoothing of special parallelepiped regions}

Here we confine ourselves to study a specially oriented parallelepiped $\Omega=$ $B^{-1}[-1,1]^{d}$ with

$$
Q_{+} \leq B^{T} B \leq c_{B} Q_{+}
$$

for a suitable $B \in \mathrm{GL}(d, \mathbb{R})$ and a positive constant $c_{B} \geq 1$ depending on $B$. In this case, the Minkowski functional of $\Omega$ is given by $M(x)=\max \left(\left\langle g_{i, \pm}, x\right\rangle\right.$ : $i=1, \ldots, d)$, where $g_{i, \pm}= \pm B^{T} e_{i}$ are $2 d$ outward normal vectors of the faces of $\Omega$. Note that the inequalities in (7.1) imply the norm equivalence

$$
d^{-1 / 2}\left\|Q_{+}^{1 / 2} x\right\| \leq M(x) \leq\left(c_{B}\right)^{1 / 2}\left\|Q_{+}^{1 / 2} x\right\| .
$$

We now approximate $I_{\Omega}$ by smooth weight functions. For this, introduce

$$
\Omega_{ \pm \varepsilon} \stackrel{\text { def }}{=}(1 \pm \varepsilon) \Omega, \quad(\partial \Omega)_{\varepsilon} \stackrel{\text { def }}{=} \Omega_{\varepsilon} \backslash \Omega_{-\varepsilon} \quad \text { and } \quad \mathrm{v}_{ \pm \varepsilon} \stackrel{\text { def }}{=} I_{\Omega_{ \pm \varepsilon}} * k_{B, \varepsilon}
$$

where $k_{B, \varepsilon}(A)=k_{\varepsilon}(B A)$ for any $A \in \mathcal{B}^{d}$ and $k_{\varepsilon}$ denotes the rescaled measure on $\mathbb{R}^{d}$ introduced in the beginning of Sect. 3.1. Moreover, we need the technical restriction $0<\varepsilon \leq \varepsilon_{0}$ with $\varepsilon_{0}:=1 / 15$. Since Lemma 3.1 can be adapted to this situation, taking $\mathrm{v}_{ \pm \varepsilon, r}(x):=\mathrm{v}_{ \pm \varepsilon}(x / r)$, we get for the lattice point remainder (3.5)

$$
\left|R\left(I_{E_{a, b} \cap r \Omega}\right)\right| \leq \max _{ \pm}\left|R\left(I_{E_{a, b}} \mathrm{v}_{ \pm \varepsilon, r}\right)\right|+R_{\varepsilon, r},
$$

where, in view of (3.2), the remainder term is given by

$$
R_{\varepsilon, r} \stackrel{\text { def }}{=} \int_{\mathbb{R}^{d}} I_{(\partial \Omega)_{2 \varepsilon}}(x / r) I_{[a, b]}(Q[x]) \mathrm{d} x .
$$

For hyperbolic shells the latter term (7.5) will be absent, but for elliptic shells we shall find that

$$
\left|R\left(I_{E_{a, b} \cap r \Omega}\right)\right| \leq \max _{ \pm}\left|R\left(I_{E_{a, b}} \mathrm{v}_{ \pm \varepsilon, r}\right)\right|+d_{Q}(b-a) \varepsilon r^{d-2} .
$$

This estimate will be proven in the following Lemma 7.1, but first we need to introduce some notations: For a measurable, non-negative, bounded weight function $\mathrm{v}$ on $\mathbb{R}^{d}$ we shall define the spherical mean by

$$
\varphi_{\mathrm{V}}\left(r_{1}, r_{2}\right) \stackrel{\text { def }}{=} \int_{S^{p-1} \times S^{q-1}} \mathrm{v}\left(Q_{+}^{-1 / 2} U^{-1}\left(r_{1} \eta_{1}, r_{2} \eta_{2}\right)\right) \mathrm{d} \sigma\left(\eta_{1}\right) \mathrm{d} \sigma\left(\eta_{2}\right),
$$


where $r_{1}, r_{2} \geq 0, \sigma$ denotes the unique normalized Haar measure on the sphere $S^{p-1}$ resp. $S^{q-1},(p, q)$ denotes the signature of $Q$ (with $p+q=d$ ) and $U$ a rotation in $\mathbb{R}^{d}$ such that $U Q U^{-1}$ is diagonal matrix whose first $p$ entries are positive and the latter $q$ are negative. Note that in the case of positive definite forms $Q$ (i.e. $q=0$ ), the double integral must be replaced by a single one.

Lemma 7.1 Let $\varphi_{\mathrm{v}}$ be defined as in (7.7). If $Q$ is indefinite, define also

$$
\|\mathrm{v}\|_{Q} \stackrel{\text { def }}{=} d_{Q} \sup _{v \in r^{-2} \partial_{w}[a, b]}\left|\int_{0}^{\infty} I\left(u^{2} \geq v\right) u^{p-1} \varphi_{\mathrm{v}}\left(u, \sqrt{u^{2}-v}\right)\left(u^{2}-v\right)^{(q-2) / 2} \mathrm{~d} u\right|
$$

and suppose that the latter integral exists. Otherwise, if $Q$ is positive definite, define

$$
\|\mathrm{v}\|_{Q} \stackrel{\text { def }}{=} d_{Q} \sup _{v \in r^{-2} \partial_{w}[a, b]}\left|v^{d-1} \varphi_{\mathrm{v}}(v)\right|
$$

and assume that the latter supremum is bounded. Under these conditions, writing $\partial_{w}[a, b]:=[a-2 w, a+2 w] \cup[b-2 w, b+2 w]$, we have for $0<w<(b-a) / 4$

$$
\int I_{\partial_{w}[a, b]}(Q[x]) \mathrm{v}(x / r) \mathrm{d} x \ll_{d} w\|\mathrm{v}\|_{Q} r^{d-2} .
$$

Assuming additionally $\max \{|a|,|b|\} \leq c_{0} r^{2}$ with $c_{0}=\left(c_{B}\right)^{-1} / 5$, the estimates

$$
\begin{aligned}
R_{\varepsilon, r} & \ll_{d} d_{Q}(b-a) \varepsilon r^{d-2} \\
\operatorname{vol} H_{r} & \gg_{d} d_{Q}\left(\sqrt{c_{B}}\right)^{-(d-2)}(b-a) r^{d-2}
\end{aligned}
$$

hold for indefinite forms $Q$, provided that $\varepsilon \in\left(0, \varepsilon_{0}\right]$. Moreover, for the special choice $\mathrm{v}=\mathrm{v}_{ \pm \varepsilon}$, as defined in (7.3), we have

$$
\left\|\mathrm{v}_{ \pm \varepsilon}\right\|_{Q} \ll_{d}|\operatorname{det} Q|^{-1 / 2}
$$

whereby the condition $\max \{|a|,|b|\} \leq c_{0} r^{2}$ can be dropped if $Q$ is positive definite.

The lower bound (7.12) can be also found in [6], see Lemma 8.2. Moreover, Lemma 3.8 in [23] provides an asymptotic formula for the volume of $H_{r}$.

Proof For a bounded measurable function $g$ on $\mathbb{R}$ with compact support we introduce

$$
R_{g} \stackrel{\text { def }}{=} \int_{\mathbb{R}^{d}} g(Q[x]) \mathrm{v}(x / r) \mathrm{d} x .
$$


Let $S_{Q}=Q Q_{+}^{-1}, L_{Q}=Q_{+}^{1 / 2}$ and let $U$ denote the rotation stated in the lemma. In particular, $U Q U^{-1}$ and $U L_{Q} U^{-1}$ are diagonal. Changing variables via $x=r L_{Q}^{-1} U^{-1} y$ in $\mathbb{R}^{d}$ with $y \in \mathbb{R}^{p} \times \mathbb{R}^{q}, d=p+q$ and using polar coordinates, $y=\left(r_{1} \eta_{1}, r_{2} \eta_{2}\right)$, where $r_{1}, r_{2}>0$ and $\eta_{1} \in S^{p-1}, \eta_{2} \in S^{q-1}$, that is $\left\|\eta_{1}\right\|=\left\|\eta_{2}\right\|=1$, we may write $Q[x]=r^{2}\left(r_{1}^{2}-r_{2}^{2}\right)$ and obtain by Fubini's theorem

$$
R_{g}=r^{d} d_{Q} \int_{0}^{\infty} \int_{0}^{\infty} r_{1}^{p-1} r_{2}^{q-1} g\left(r^{2}\left(r_{1}^{2}-r_{2}^{2}\right)\right) \varphi_{\mathrm{v}}\left(r_{1}, r_{2}\right) \mathrm{d} r_{1} \mathrm{~d} r_{2}
$$

where $\varphi_{\mathrm{v}}\left(r_{1}, r_{2}\right)$ is defined as in (7.7) for suitable weight functions v. (As already noted, in the case of positive definite forms $Q$, the double integral in (7.14) must be replaced by a single one.) Next, we change variables via $v:=r_{1}^{2}-r_{2}^{2}$ and $u:=r_{1}$, so that $r_{1}^{2}+r_{2}^{2}=2 u^{2}-v$ and $r_{2}=\sqrt{u^{2}-v}$. Thus, we get

$$
R_{g}=r^{d} \frac{d Q}{2} \int_{\mathbb{R}} g\left(r^{2} v\right) \int_{0}^{\infty} I\left(u^{2} \geq v\right) u^{p-1} \varphi_{\mathrm{v}}\left(u, \sqrt{u^{2}-v}\right)\left(u^{2}-v\right)^{(q-2) / 2} \mathrm{~d} u \mathrm{~d} v .
$$

In order to prove (7.10), we choose $g=I_{\partial_{w}[a, b]}$ in (7.15). Since the length of $r^{-2} \operatorname{supp} g$ is at most $\ll|w| r^{-2}$, we get $R_{g} \ll_{d}|w| r^{d-2}\|\mathrm{v}\|_{Q}$, where $\|\mathrm{v}\|_{Q}$ is defined as in (7.8) if $Q$ is indefinite, resp. as in (7.9) if $Q$ is positive definite.

Next we prove (7.12): Taking $g=I_{[a, b]}, \mathrm{v}(x)=I_{\Omega}(x)=I(M(x) \leq 1)$ and using

$$
\|y\| d^{-1 / 2} \leq M\left(L_{Q}^{-1} U^{-1} y\right) \leq\|y\|\left(c_{B}\right)^{1 / 2}
$$

gives the lower bound

$$
\begin{aligned}
\varphi_{\mathrm{v}}\left(r_{1}, r_{2}\right) & \geq \int_{S^{p-1} \times S^{q-1}} I\left(\left\|\left(r_{1} \eta_{1}, r_{2} \eta_{2}\right)\right\| \leq\left(c_{B}\right)^{-1 / 2}\right) \mathrm{d} \sigma\left(\eta_{1}\right) \mathrm{d} \sigma\left(\eta_{2}\right) \\
& \gg_{d} I\left(2 u^{2}+|v| \leq\left(c_{B}\right)^{-1}\right) .
\end{aligned}
$$

Thus, we find

$$
\begin{aligned}
\operatorname{vol} H_{r} & \gg_{d} r^{d} d_{Q} \int_{r^{-2} a}^{r^{-2} b} \int_{0}^{\infty} I\left(u^{2} \geq v\right) I\left(2 u^{2}+|v| \leq\left(c_{B}\right)^{-1}\right) u^{p-1}\left(u^{2}-v\right)^{\frac{(q-2)}{2}} \mathrm{~d} u \mathrm{~d} v \\
& \gg_{d} r^{d} d_{Q} \int_{r^{-2} a}^{r^{-2} b} I\left(|v| \leq c_{0}\right) \int_{0}^{\infty} I\left(\frac{5}{4} c_{0} \leq u^{2} \leq 2 c_{0}\right) u^{p-1}\left(u^{2}-v\right)^{\frac{q-2}{2}} \mathrm{~d} u \mathrm{~d} v \\
& \gg_{d} r^{d-2}(b-a) d_{Q}\left(\sqrt{c_{0}}\right)^{d-2} .
\end{aligned}
$$

Proof of (7.11) In (7.15) we choose $g=I_{[a, b]}$ and $\mathrm{v}=I_{(\partial \Omega)_{2 \varepsilon}}$ with $0<\varepsilon \leq$ $\varepsilon_{0}$. By the properties of the polyhedron $\Omega$, see (7.2), we have $I_{(\partial \Omega)_{2 \varepsilon}}(x) \leq$ 
$I\left(M(x) \in J_{1,2 \varepsilon}\right)$, where $J_{1,2 \varepsilon}:=[1-2 \varepsilon, 1+2 \varepsilon]$. Let $g_{1}, \ldots, g_{2 d}$ denote the $2 d$-tuple of normal vectors defining $\Omega$ and let $f_{m}=U L_{Q}^{-1} g_{m}, m=1, \ldots, 2 d$, be the transformed vectors. Since

$$
I\left(M\left(L_{Q}^{-1} U^{-1} y\right) \in J_{1,2 \varepsilon}\right) \leq \sum_{m=1}^{2 d} I\left(\left\langle y, f_{m}\right\rangle \in J_{1,2 \varepsilon}\right)
$$

we may bound $\varphi_{\mathrm{v}}\left(r_{1}, r_{2}\right)$ in (7.15) as follows

$$
\varphi_{\mathrm{v}}\left(r_{1}, r_{2}\right) \leq \sum_{m=1}^{2 d} \varphi_{\mathrm{v}, m}\left(r_{1}, r_{2}\right),
$$

where

$$
\varphi_{\mathrm{v}, m}\left(r_{1}, r_{2}\right) \stackrel{\text { def }}{=} \int_{S^{p-1} \times S^{q-1}} I\left[\left\langle\left(r_{1} \eta_{1}, r_{2} \eta_{2}\right), f_{m}\right\rangle \in J_{1,2 \varepsilon}\right] \mathrm{d} \eta_{1} \mathrm{~d} \eta_{2} .
$$

Recall $|v| \leq c_{0}, v=r_{1}^{2}-r_{2}^{2}, u=r_{1}$ and $r_{2}=\sqrt{u^{2}-v}$. The inequality (7.16) implies

$$
(1+2 \varepsilon)^{2} d \geq r_{1}^{2}+r_{2}^{2}=2 u^{2}-v \geq(1-2 \varepsilon)^{2}\left(c_{B}\right)^{-1} .
$$

Therefore $\varphi_{\mathrm{v}}\left(u, \sqrt{u^{2}-v}\right)=0$ if

$$
0 \leq u<2^{-\frac{1}{2}} \sqrt{5 c_{0}(1-2 \varepsilon)^{2}-c_{0}} \quad \text { or } \quad u>C_{\Omega} \stackrel{\text { def }}{=} \frac{(1+2 \varepsilon)}{\sqrt{2}} \sqrt{d+c_{0}} .
$$

Because of

$$
2^{-\frac{1}{2}} \sqrt{5 c_{0}(1-2 \varepsilon)^{2}-c_{0}} \geq c_{\Omega} \stackrel{\text { def }}{=} \frac{\sqrt{310 c_{0}}}{15}
$$

and $u^{2}-v \geq 17 c_{0} / 45>0$, we get

$$
\begin{aligned}
R_{g} & \ll r^{d} d_{Q} \int_{r^{-2} a}^{r^{-2} b}\left(\int_{c_{\Omega}}^{C_{\Omega}} u^{p-1}\left(u^{2}-v\right)^{\frac{q-2}{2}} \varphi_{\mathrm{v}}\left(u, \sqrt{u^{2}-v}\right) \mathrm{d} u\right) \mathrm{d} v \\
& \leq r^{d} d_{Q} \sum_{m=1}^{2 d} \int_{r^{-2} a}^{r^{-2} b}\left(\int_{c_{\Omega}}^{C_{\Omega}} u^{p-1}\left(u^{2}-v\right)^{\frac{q-2}{2}} \varphi_{\mathrm{v}, m}\left(u, \sqrt{u^{2}-v}\right) \mathrm{d} u\right) \mathrm{d} v .
\end{aligned}
$$

By interchanging the variables $r_{1}$ and $r_{2}$ we can suppose that $q \geq 2$. Thus, since $u \ll_{d} 1$ and $\sqrt{u^{2}-v} \ll_{d} 1$, we see that

$$
\int_{c_{\Omega}}^{C_{\Omega}} u^{p-1}\left(u^{2}-v\right)^{\frac{q-2}{2}} \varphi_{\mathrm{v}, m}\left(u, \sqrt{u^{2}-v}\right) \mathrm{d} u \ll_{d} \int_{c_{\Omega}}^{C_{\Omega}} \varphi_{\mathrm{v}, m}\left(u, \sqrt{u^{2}-v}\right) \mathrm{d} u .
$$


We claim that

$$
R_{g} \ll_{d} d_{Q} \varepsilon(b-a) r^{d-2}
$$

holds. In view of (7.17) and (7.18), the estimates

$$
R_{m} \stackrel{\text { def }}{=} \int_{c_{\Omega}}^{C_{\Omega}} \varphi_{\mathrm{v}, m}\left(u, \sqrt{u^{2}-v}\right) \mathrm{d} u \ll_{d} \varepsilon c_{\Omega}
$$

for all $m=1, \ldots, 2 d$ will prove the bound (7.19).

Thus let $F_{m}(u):=\left\langle\left(u \eta_{1},\left(u^{2}-v\right)^{1 / 2} \eta_{2}\right), f_{m}\right\rangle$ for fixed $|v| \leq c_{0}$ and $\left(\eta_{1}, \eta_{2}\right)$. If

$$
\left|\frac{\partial}{\partial u} F_{m}(u)\right| \geq c_{1}>0
$$

for all $c_{\Omega} \leq u \leq C_{\Omega}$ with $F_{m}(u) \in[1-2 \varepsilon, 1+2 \varepsilon]$ uniformly in $\left(\eta_{1}, \eta_{2}\right)$ and $v$, then

$$
\int_{c_{\Omega}}^{C_{\Omega}} I\left(F_{m}(u) \in[1-2 \varepsilon, 1+2 \varepsilon]\right) \mathrm{d} u \ll \frac{\varepsilon}{c_{1}}
$$

and hence $R_{m} \ll_{d} c_{1}^{-1} \varepsilon$ for all $m=1, \ldots, 2 d$. Note that

$$
\frac{\partial}{\partial u} F_{m}(u)=\frac{1}{u}\left(F_{m}(u)+\frac{v}{\sqrt{u^{2}-v}}\left\langle\left(0, \eta_{2}\right), f_{m}\right\rangle\right)
$$

and because of $\left\|L_{Q}^{-1} B^{T}\right\|=\left\|B L_{Q}^{-1}\right\| \leq \sqrt{c_{B}}$ we see that

$$
\left|\frac{\partial}{\partial u} F_{m}(u)\right| \geq \frac{1}{u}\left(\left|F_{m}(u)\right|-\frac{c_{0}}{\sqrt{17 c_{0} / 45}}\left\|f_{m}\right\|\right) \geq \frac{1}{u}\left(1-2 \varepsilon-\frac{4}{5}\right) \gg c_{\Omega}^{-1} .
$$

Note, that here it is important that $\varepsilon>0$ is not too large, i.e. $\varepsilon \in\left(0, \varepsilon_{0}\right]$. Thus, (7.20) holds and the assertion (7.19) is proved. This yields the claimed bound for $R_{\varepsilon, r}$, compare (7.5).

Finally, we prove (7.13). Here we have $v=v_{ \pm \varepsilon}$ and $v_{ \pm \varepsilon}(x) \leq I(M(x) \leq$ $1+2 \varepsilon$ ). In view of (7.16), we find that the $u$-integral in (7.8) can be restricted to $2 u^{2} \leq 2 d+v$. Hence

$$
\left\|\mathrm{v}_{ \pm \varepsilon}\right\|_{Q} \ll{ }_{d} d_{Q} \sup _{v \in r^{-2} \partial_{w}[a, b]}(1+|v|)^{(d-3) / 2} \int_{0}^{\infty} I\left(v \leq u^{2} \leq d+v / 2\right) \mathrm{d} u \ll d_{Q},
$$


because $|v| \leq r^{-2}(|a|+|b|) \leq 2 c_{0} \leq 1$. Since $\varphi_{\mathrm{v}}$ is supported in $\|\cdot\|$-ball of radius $2 d^{1 / 2}$, we get also in the case of positive definite forms that (7.9) is bounded by $\ll_{d} d_{Q}$.

\subsection{Fourier transform of weights for polyhedra}

Here we continue to estimate the remainder terms in (7.6). Since the bounds for $R\left(g_{w}^{Q} \mathrm{v}_{-\varepsilon, r}\right)$ are exactly the same as for $R\left(g_{w}^{Q} \mathrm{v}_{+\varepsilon, r}\right)$ we shall consider the latter only. We shall now modify the weight $\mathrm{v}_{\varepsilon}$, defined in (7.3), as follows. Define $\varphi=I_{[-2,2]} * k$, where $k$ is again the probability measure from Sect. 3.1. Of course, $\varphi$ is smooth and $\varphi(u)=1$ if $|u| \leq 1$ and $\varphi(u)=0$ if $|u| \geq 3$. Let $s_{d}:=d\left(1+2 \varepsilon_{0}\right)^{2}$. Now, by construction $\varphi\left(Q_{+}[x] s_{d}^{-1}\right)$ is identical to 1 on the support of the $\varepsilon$-smoothed indicator of $\Omega_{\varepsilon}=B^{-1}[-(1+\varepsilon),(1+\varepsilon)]^{d}$, that is $\mathrm{v}_{\varepsilon}(x)$. Hence we may rewrite the weights $\zeta$ of (3.6) via

$$
\zeta_{\varepsilon}(x)=\mathrm{v}_{\varepsilon}(x) \exp \left\{Q_{+}[x]\right\}=\mathrm{v}_{\varepsilon}(x) \psi(x)
$$

using the $C^{\infty}$ function $\psi(x):=\exp \left\{Q_{+}[x]\right\} \varphi\left(Q_{+}[x] s_{d}^{-1}\right)$ of bounded support, whose Fourier transform can easily be estimated, see (7.24). In particular, the weights $\zeta_{\varepsilon}$ satisfy the integrability condition (2.4), i.e. $\sup _{x \in \mathbb{R}^{d}}\left(\left|\zeta_{\varepsilon}(x)\right|+\right.$ $\left.\widehat{\zeta}_{\varepsilon}(x) \mid\right)(1+\|x\|)^{d+1}<\infty$.

Lemma 7.2 The following estimate holds

$$
\int_{\mathbb{R}^{d}}\left|\widehat{\xi_{\varepsilon}}(v)\right| \mathrm{d} v \ll_{d} \int\left|\widehat{I}_{[-1,1]^{d}}\right|(v) \prod_{j=1}^{d} \exp \left\{-\left|\varepsilon v_{j}\right|^{1 / 2}\right\} \mathrm{d} v \ll_{d}\left(\log \varepsilon^{-1}\right)^{d} .
$$

Remark 7.3 In the general case, when $\Omega$ has finite Minkowski surface measure $c_{\Omega}$ only, defined via meas $\left(\partial_{\varepsilon} \Omega\right) \leq c_{\Omega} \varepsilon$, we have

$$
\left\|\widehat{I_{\Omega}}\right\|_{1, \varepsilon} \stackrel{\text { def }}{=} \int_{\mathbb{R}^{d}}\left|\widehat{I}_{\Omega}(v)\right| \exp \left\{-\|\varepsilon v\|^{1 / 2}\right\} \mathrm{d} v \ll_{d} c_{\Omega} \varepsilon^{-d}
$$

as can be deduced from the bound in Theorem 2.9 of [2], that is

$$
\frac{1}{\operatorname{vol}(u \leq\|v\| \leq 2 u)} \int_{\{u \leq\|v\| \leq 2 u\}}\left|\widehat{I}_{\Omega}(v)\right| \mathrm{d} v \leq c_{\Omega}(2+u)^{-(d+1) / 2} .
$$

This estimate is sharp as shown by the explicit example of an unit ball, see [2] for more details. That paper contains also bounds on the average $\eta \mapsto\left|\widehat{I}_{\Omega}(s \eta)\right|$ over the unit sphere $S^{d-1}$ for polyhedra, which are usually of smaller order than pointwise bounds. In fact, the pointwise decay of $\widehat{I}_{\Omega}(v)$ may depend crucially on the direction of $v$. In our setting (finding $L^{1}$-estimates for specially oriented parallelepipeds $\Omega$ ) more elementary arguments can be used. 
Proof Note that by definition

$$
\int_{\mathbb{R}^{d}}\left|\widehat{\zeta_{\varepsilon}}(v)\right| \mathrm{d} v=\int_{\mathbb{R}^{d}} \widehat{\mid \mathrm{v}_{\varepsilon} \psi}(v)\left|\mathrm{d} v=\int_{\mathbb{R}^{d}}\right| \int_{\mathbb{R}^{d}} \widehat{\widehat{\varepsilon}}(v-x) \widehat{\psi}(x) \mathrm{d} x \mid \mathrm{d} v \leq\left\|\widehat{\varepsilon_{\varepsilon}}\right\|_{1}\|\widehat{\psi}\|_{1} .
$$

Since

$$
\widehat{\psi}(x)=|\operatorname{det} Q|^{-1 / 2} \int_{\mathbb{R}^{d}} \exp \left[v^{2}\right] \varphi\left(v^{2} s_{d}^{-1}\right) \mathrm{e}^{-2 \pi \mathrm{i}\left\langle v, Q_{+}^{-1 / 2} x\right\rangle} \mathrm{d} v
$$

we easily conclude that

$$
|\widehat{\psi}(x)| \leq|\operatorname{det} Q|^{-1 / 2} c(d, k)\left(1+Q_{+}^{-1}[x]\right)^{-k}, x \in \mathbb{R}^{d}, \text { and thus }\|\widehat{\psi}\|_{1} \leq c(d) . \text { (7.24) }
$$

Defining $Z:=\left(B^{-1}\right)^{T}$ and changing variables shows also that

$$
\widehat{I}_{\Omega_{\varepsilon}}(v)=(1+\varepsilon)^{d} \widehat{I}_{\Omega}((1+\varepsilon) v)=(1+\varepsilon)^{d}|\operatorname{det} B|^{-1} \widehat{I}_{[-1,1]^{d}}((1+\varepsilon) Z v)
$$

and

$$
\left|\widehat{k}_{B, \varepsilon}(v)\right| \leq \exp \left\{-\varepsilon^{1 / 2} \sum_{j=1}^{d}\left|(Z v)_{j}\right|^{1 / 2}\right\} .
$$

Thus we get for $\mathrm{v}_{\varepsilon}=I_{\Omega_{\varepsilon}} * k_{B, \varepsilon}$

$$
\left\|\widehat{v}_{\varepsilon}\right\|_{1}=\left\|\widehat{I}_{\Omega_{\varepsilon}} \widehat{k}_{B, \varepsilon}\right\|_{1} \ll_{d} \int_{\mathbb{R}^{d}}\left|\widehat{I}_{[-1,1]^{d}}((1+\varepsilon) v)\right| \prod_{j=1}^{d} \exp \left\{-\left|\varepsilon v_{j}\right|^{1 / 2}\right\} \mathrm{d} v .
$$

Finally, using $\widehat{I}_{[-1,1]^{d}}(v)=\prod_{j=1}^{d} \sin \left(2 \pi v_{j}\right) /\left(\pi v_{j}\right)$ together with (7.27) gives the estimate

$$
\left\|\widehat{\mathrm{v}}_{\varepsilon}\right\|_{1} \ll_{d}\left(\int_{0}^{\infty} \frac{1}{u+\varepsilon} \mathrm{e}^{-\sqrt{u}} \mathrm{~d} u\right)^{d} \ll_{d}\left(1+\int_{0}^{1} \frac{1}{u+\varepsilon} \mathrm{d} u\right)^{d} \ll_{d} \log \left(\varepsilon^{-1}\right)^{d} .
$$

We now obtain the estimate (7.22) from (7.23) combined with (7.24) and (7.28).

\subsection{Lattice point remainders for admissible parallelepipeds}

Now we restrict the parallelepiped $\Omega=B^{-1}[-1,1]^{d}$, as defined in (7.1), such that its faces are in a general position relative to the standard lattice $\mathbb{Z}^{d}$. This ensures that the lattice point remainder for $r \Omega$ is of 'abnormally' small error uniformly in $r$. To construct it, we may alternatively construct lattices $B \mathbb{Z}^{d}$ 
such that the faces of $[-1,1]^{d}$ have this property. Following Skriganov [53], we call a lattice $\Gamma \subset \mathbb{R}^{d}$ of full rank, and likewise $\Omega$, 'admissible' if

$$
\mathrm{Nm} \Gamma \stackrel{\text { def }}{=} \inf _{\gamma \in \Gamma \backslash\{0\}}|\mathrm{Nm} \gamma|>0,
$$

where $\operatorname{Nm} \gamma=\left|\gamma_{1} \cdots \gamma_{d}\right|$ in standard coordinates $\gamma=\left(\gamma_{1}, \ldots, \gamma_{d}\right)$.

Remark 7.4 The set of all admissible lattices is dense in the space of lattices (see [54]). Hence, for any $\eta>0$, if $D_{\eta}$ denotes the set of diagonal matrices with entries in $[1,1+\eta)$, then $\mathrm{O}(d) D_{\eta} \mathrm{O}(d) \Gamma$ contains an admissible lattice. In particular, if $\Gamma=Q_{+}^{1 / 2} \mathbb{Z}^{d}$, then there exist orthogonal matrices $k, l \in \mathrm{O}(d)$ and a diagonal matrix $d \in D_{\eta}$ such that $B \mathbb{Z}^{d}$ is admissible, where $B=k d l Q_{+}^{1 / 2}$ satisfies property (7.1) with a constant $c_{B}$ depending only on $\eta$.

Remark 7.5 This definition is a special case of 'admissible lattices' for starbodies, see Chapter IV.4 in [14]. Here, the star-body is given by $\{F<1\}$ with the distance function $F(x)=\left|x_{1} \cdots x_{d}\right|^{1 / d}$.

As shown in Lemma 3.1 of [53], the dual lattice $\Gamma^{*}=Z \mathbb{Z}^{d}$ of $\Gamma$, where $Z^{T} B=\mathrm{Id}$, is admissible as well. Another property of admissible lattices is that there exists a cube $\left[-r_{0}, r_{0}\right]^{d}$ containing a fundamental domain $F$ of $\Gamma$ such that $r_{0}>0$ depends only by means of the invariants det $\Gamma$ and $\mathrm{Nm} \Gamma$.

Example 7.6 Well known examples are provided by the Minkowski embedding of a totally real algebraic number field $\mathbb{F}$ of degree $d$ into $\mathbb{R}^{d}$. Given all embeddings $\sigma_{1}, \ldots, \sigma_{d}$ of $\mathbb{F}$, the Minkowski embedding $\sigma: \mathbb{F} \rightarrow \mathbb{R}^{d}$ is defined by $\sigma=\left(\sigma_{1}, \ldots, \sigma_{d}\right)$. In this case $\operatorname{Nm} \sigma(\alpha)=\left|N_{\mathbb{F} / \mathbb{Q}}(\alpha)\right|$ is the field norm of any $\alpha \in \mathbb{F}$, where we interpret multiplication by $\alpha$ as a $\mathbb{Q}$-linear map. Thus, the image of the ring of integers $\mathcal{O}_{\mathbb{F}}$ is an admissible lattice $\Gamma$ with $\mathrm{Nm} \Gamma \geq 1$. For more information, see Chapter 2.3 in [11].

Remark 7.7 We also note that for any natural number $n \in \mathbb{N}$ we may choose a real number field of degree $n$ which is normal over the rational numbers. In fact, let $m \in \mathbb{N}$ be chosen such that $2 n \mid \varphi(m)$ and let $\xi_{m}$ be a primitive $m$-th root of unity. Then $\mathbb{Q}\left(\xi_{m}+\xi_{m}^{-1}\right)$ is a real number field of degree $\varphi(m) / 2$, which is also normal and its Galois group $G$ is abelian. Since $G$ contains a subgroup $H$ of order $\varphi(m) /(2 n)$, the fixed field of $H$ is real, normal and of degree $n$. Thus, there exists an admissible region $\Omega$ satisfying (7.1) with $c_{B} \asymp_{d} q / q_{0}$ and $\operatorname{Nm}(B) \asymp_{d} q^{d / 2}$.

Lemma 7.8 Assume that the lattice $\Gamma=B \mathbb{Z}^{d}$ is admissible and $B$ satisfies (7.1). For $0<\varepsilon \leq \varepsilon_{0}$ and $r \geq 1$ we get for the parallelepiped 
$\Omega=B^{-1}[-1,1]^{d}$ and the corresponding weights $\zeta_{\varepsilon}(x)=\mathrm{v}_{\varepsilon}(x) \psi(x)$ introduced in Sect. 7.2

$$
I_{\zeta} \stackrel{\text { def }}{=} \int_{\|v\|_{\infty}>r / 2} \frac{\left|\widehat{\zeta}_{\varepsilon}(v)\right|}{\left(q^{1 / 2} r^{-1}+\left\|r^{-1} v\right\|_{\mathbb{Z}^{d}}\right)^{d / 2}} \mathrm{~d} v \ll_{d} q_{0}^{-d / 4} d_{Q}|\operatorname{det} B| \lambda_{r, \varepsilon}^{d-1} \frac{\bar{\lambda}_{r, \varepsilon, \Gamma}}{\operatorname{Nm}(\Gamma)},
$$

where $\lambda_{r, \varepsilon}:=\min \left\{\log (r+1), \log \left(\varepsilon^{-1}\right)\right\}$ and $\bar{\lambda}_{r, \varepsilon, \Gamma}:=\max \left\{\lambda_{r, \varepsilon}, \log (2+\right.$ $\left.\left.\frac{1}{\operatorname{Nm}(\Gamma) r \varepsilon}\right)\right\}$. For any non-admissible parallelepiped $\Omega$ only the estimate

$$
I_{\zeta} \ll d_{Q} q^{d / 2} c_{B}^{(d+1) / 2} \varepsilon^{-d}
$$

holds. Additionally, we also have $d_{Q}|\operatorname{det} B| \leq\left(c_{B}\right)^{d / 2}$.

Proof We start by making the change of variables $w=r^{-1} Z v$ in (7.30) and then splitting $I_{\zeta}$ into integrals over cells $C^{*}:=Z\left[-\frac{1}{2}, \frac{1}{2}\right)^{d}$, where $\Gamma^{*}:=Z \mathbb{Z}^{d}$ denotes the dual lattice to $\Gamma$, that is $Z=\left(B^{T}\right)^{-1}$, in order to get

$$
I_{\zeta}=\sum_{\gamma^{*} \in \Gamma^{*} \backslash\{0\}} I_{\zeta}\left(\gamma^{*}\right), \text { where } I_{\zeta}(m) \stackrel{\text { def }}{=} r^{d}|\operatorname{det} B| \int_{C^{*}} \frac{\widehat{\mid \zeta} \varepsilon\left(Z^{-1} r\left(\gamma^{*}+v\right)\right) \mid}{\left(q^{\frac{1}{2}} r^{-1}+\left\|Z^{-1} v\right\|_{\infty}\right)^{\frac{d}{2}}} \mathrm{~d} v .
$$

Note that $\Gamma^{*}$ satisfies $\|Z\| \leq\left\|Q_{+}^{-1 / 2}\right\| \leq q_{0}^{-1 / 2}$, since the first inequality in (7.1) implies

$$
1 \geq\left\|Q_{+}^{1 / 2} B^{-1}\right\|=\left\|\left(\left(B^{T}\right)^{-1} Q_{+}^{1 / 2}\right)^{T}\right\|=\left\|\left(B^{T}\right)^{-1} Q_{+}^{1 / 2}\right\|=\left\|Z Q_{+}^{1 / 2}\right\| .
$$

In particular, the fundamental domain $C^{*}$ is contained in $q_{0}^{-1 / 2} \sqrt{d}\left[-\frac{1}{2}, \frac{1}{2}\right]^{d}$. Next, we shall bound the Fourier transform of $\zeta_{\varepsilon}$. Recall that by definition

$$
\widehat{\zeta}_{\varepsilon}(u)=\left(\left(\widehat{I}_{\Omega_{\varepsilon}} \cdot \widehat{k}_{B, \varepsilon}\right) * \widehat{\psi}\right)(u) .
$$

As verified in (7.25), we have in coordinates $u=\left(u_{1}, \ldots, u_{d}\right)$

$$
\left|\widehat{I}_{\Omega_{\varepsilon}}\left(Z^{-1} u\right)\right| \ll_{d}|\operatorname{det} B|^{-1} \prod_{j=1}^{d}\left|\frac{\sin \left[2 \pi(1+\varepsilon) u_{j}\right]}{(1+\varepsilon) u_{j}}\right| \ll_{d}|\operatorname{det} B|^{-1} \prod_{j=1}^{d}\left(1+\left|u_{j}\right|\right)^{-1} .
$$

Since (7.33) also implies $\left\|Q_{+}^{-1 / 2}\left(Z^{-1} u\right)\right\| \geq\|u\|$, we can rewrite (7.24) by

$$
\left|\widehat{\psi}\left(Z^{-1} u\right)\right| \ll_{d, k}|\operatorname{det} Q|^{-1 / 2}\left(1+\|u\|^{2}\right)^{-k} \ll_{d, k}|\operatorname{det} Q|^{-1 / 2} \prod_{j=1}^{d}\left(1+u_{j}^{2}\right)^{-k / d},
$$

where we applied the AM-GM inequality. In view of (7.26) we have the bound

$$
\left|\widehat{k}_{B, \varepsilon}\left(Z^{-1} u\right)\right| \leq \exp \left\{-\sum_{j=1}^{d}\left|\varepsilon u_{j}\right|^{1 / 2}\right\}
$$


as well. Combining these estimates yields

$$
\left|\widehat{\zeta}_{\varepsilon}\left(Z^{-1} r w\right)\right| \ll_{d, k} d_{Q} \int_{\mathbb{R}^{d}} \prod_{j=1}^{d} \frac{1}{\left(1+u_{j}^{2}\right)^{k / d}} \frac{\exp \left\{-\varepsilon^{1 / 2}\left|r w_{j}-u_{j}\right|^{1 / 2}\right\}}{1+\left|r w_{j}-u_{j}\right|} \mathrm{d} u .
$$

Thus, we get for a fixed lattice point $\gamma^{*}=\left(\gamma_{1}^{*}, \ldots, \gamma_{d}^{*}\right) \in \Gamma^{*}$

$$
I_{\zeta}\left(\gamma^{*}\right) \ll \ll_{d, k} \int_{C^{*}} \frac{|\operatorname{det} Q|^{-1 / 2}|\operatorname{det} B|}{\left(q r^{-1}+\left\|Z^{-1} v\right\|_{\infty}\right)^{d / 2}} \int_{\mathbb{R}^{d}} \prod_{j=1}^{d} \bar{\omega}\left(u_{j}\right) \frac{\omega\left(\varepsilon r\left(\gamma_{j}^{*}+v_{j}-\frac{u_{j}}{r}\right)\right)}{r^{-1}+\left|\gamma_{j}^{*}+v_{j}-\frac{u_{j}}{r}\right|} \mathrm{d} u \mathrm{~d} v,
$$

where $\bar{\omega}(x):=\left(1+x^{2}\right)^{-k / d}$ and $\omega(x):=\exp \left\{-|x|^{1 / 2}\right\}$. We now estimate the last double integral coordinatewise: Note that we have $\left|v_{i}\right| \leq \bar{v}:=\sqrt{d} / 2$ and

$$
\left(q^{1 / 2} r^{-1}+\left\|Z^{-1} v\right\|_{\infty}\right)^{d / 2} \gg_{d} q_{0}^{d / 4}\left(r^{-1}+\|v\|_{\infty}\right)^{d / 2} \geq q_{0}^{d / 4} \prod_{j=1}^{d}\left(r^{-1}+\left|v_{i}\right|\right)^{1 / 2},
$$

since $\left\|Z^{-1} v\right\|_{\infty} \gg_{d}\|Z\|^{-1}\|v\|_{\infty} \geq q_{0}^{1 / 2}\|v\|_{\infty}$. Hence, we find

$$
I_{\zeta}\left(\gamma^{*}\right) \ll_{d, k} q_{0}^{-d / 4} d_{Q}|\operatorname{det} B| \prod_{j=1}^{d} J_{\zeta}\left(\gamma_{j}^{*} ; \mathbb{R}\right),
$$

where

$$
J_{\zeta}\left(\gamma_{j}^{*} ; D\right) \stackrel{\text { def }}{=} \int_{-\bar{v}}^{\bar{v}} \frac{1}{\left(r^{-1}+|v|\right)^{1 / 2}} \int_{D} \bar{\omega}(u) \frac{\omega\left(\varepsilon r\left(\gamma_{j}^{*}+v-\frac{u}{r}\right)\right)}{r^{-1}+\left|\gamma_{j}^{*}+v-\frac{u}{r}\right|} \mathrm{d} u \mathrm{~d} v .
$$

In order to estimate $J_{\zeta}\left(\gamma_{j}^{*} ; \mathbb{R}\right)$, we decompose the integral into parts corresponding to the extremal points of the integrands. Defining $D_{j}:=\{|u| \geq$ $\left.r\left|\gamma_{j}^{*}+v\right| / 2\right\}$, we get

$$
J_{\zeta}\left(\gamma_{j}^{*} ; D_{j}\right) \leq \int_{-\bar{v}}^{\bar{v}} \frac{r}{|v|^{1 / 2}} \int_{D_{j}} \bar{\omega}(u) \mathrm{d} u \mathrm{~d} v \ll k, d \int_{-\bar{v}}^{\bar{v}} \frac{1}{|v|^{1 / 2}} \frac{r}{\left(1+r\left|\gamma_{j}^{*}+v\right|\right)^{\frac{k}{d}-1}} \mathrm{~d} v .
$$

In the case $\left|\gamma_{j}^{*}\right| \geq \sqrt{d}$, we have $\left|\gamma_{j}^{*}+v\right| \geq\left|\gamma_{j}^{*}\right| / 2$ and hence

$$
J_{\zeta}\left(\gamma_{j}^{*} ; D_{j}\right) \ll_{d} \frac{r}{\left(1+\left|r \gamma_{j}^{*}\right|\right)^{d+2}} \int_{-\bar{v}}^{\bar{v}} \frac{1}{|v|^{1 / 2}} \mathrm{~d} v \ll_{d} \frac{1}{\left(1+\left|r \gamma_{j}^{*}\right|\right)^{d+1}}
$$


if we take $k=d(d+3)$. In the other case $\left|\gamma_{j}^{*}\right|<\sqrt{d} / 2$, we split the $v$-integral into two parts as follows in order to find the estimate

$$
\begin{aligned}
J_{\zeta}\left(\gamma_{j}^{*} ; D_{j}\right) & \ll_{d} \int_{-\bar{v}}^{\bar{v}} \frac{\left|\gamma_{j}^{*}\right|^{-\frac{1}{2}} r I\left(|v| \geq\left|\gamma_{j}^{*}\right| / 2\right)}{\left(1+r\left|\gamma_{j}^{*}+v\right|\right)^{d+2}} \mathrm{~d} v+\int_{0}^{\left|\gamma_{j}^{*}\right| / 2} \frac{r}{v^{\frac{1}{2}}\left(1+r\left(\left|\gamma_{j}^{*}\right|-v\right)\right)^{d+2}} \mathrm{~d} v \\
& \ll_{d}\left|\gamma_{j}^{*}\right|^{-\frac{1}{2}}+\frac{\left|\gamma_{j}^{*}\right|^{\frac{1}{2}} r}{\left(r\left|\gamma_{j}^{*}\right|+1\right)^{d+2}} \int_{0}^{1 / 2} \frac{1}{v^{\frac{1}{2}}(1-v)^{d+2}} \mathrm{~d} v \ll_{d}\left|\gamma_{j}^{*}\right|^{-\frac{1}{2}} .
\end{aligned}
$$

In the complement $u \in D_{j}^{c}$ we have $\left|\gamma_{j}^{*}+v-\frac{u}{r}\right| \geq\left|\gamma_{j}^{*}+v\right| / 2$ and thus

$$
J_{\zeta}\left(\gamma_{j}^{*} ; D_{j}^{c}\right) \ll_{d} \int_{-\bar{v}}^{\bar{v}}|v|^{-\frac{1}{2}} \frac{\omega\left(\varepsilon r\left(\gamma_{j}^{*}+v\right) / 2\right)}{r^{-1}+\left|\gamma_{j}^{*}+v\right|} \mathrm{d} v .
$$

If $\left|\gamma_{j}^{*}\right| \geq \sqrt{d}$, then we easily conclude that $J_{\zeta}\left(\gamma_{j}^{*} ; D_{j}^{c}\right) \ll_{d} \omega\left(\varepsilon r \gamma_{j}^{*} / 4\right)\left|\gamma_{j}^{*}\right|^{-1}$. At last, we consider the case $\left|\gamma_{j}^{*}\right|<\sqrt{d}$. The $v$-integral over the region $\left\{\bar{v} \geq|v| \geq\left|\gamma_{j}^{*}\right| / 2\right\}$ can be bounded by

$$
\begin{aligned}
& \ll_{d}\left|\gamma_{j}^{*}\right|^{-1 / 2} \int_{-\bar{v}}^{\bar{v}} \frac{I\left(|v| \geq\left|\gamma_{j}^{*}\right| / 2\right)}{\left(r^{-1}+\left|\gamma_{j}^{*}+v\right|\right)\left(1+\varepsilon r\left|\gamma_{j}^{*}+v\right|\right)} \mathrm{d} v \\
& \ll_{d}\left|\gamma_{j}^{*}\right|^{-1 / 2} \int_{0}^{3 \sqrt{d} / 2} \frac{1}{r^{-1}+v} \frac{1}{1+\varepsilon r v} \mathrm{~d} v \ll_{d}\left|\gamma_{j}^{*}\right|^{-1 / 2} \min \left\{\log \left(\varepsilon^{-1}\right), \log (r+1)\right\}
\end{aligned}
$$

and similar over the complement by

$$
\ll_{d} \int_{0}^{\left|\gamma_{j}^{*}\right| / 2} \frac{v^{-1 / 2}}{r^{-1}+\left|\gamma_{j}^{*}\right|-v} \mathrm{~d} v \ll_{d}\left|\gamma_{j}^{*}\right|^{-1 / 2} .
$$

Hence we conclude that

$$
I_{\zeta} \ll_{d} q_{0}^{-d / 4} d_{Q}|\operatorname{det} B| \sum_{\left(\gamma_{1}^{*}, \ldots, \gamma_{d}^{*}\right) \in \Gamma^{*} \backslash\{0\}} \prod_{j=1}^{d} \frac{H_{r, \varepsilon}\left(\gamma_{j}^{*}\right)}{\left|\gamma_{j}^{*}\right|},
$$

where

$$
H_{r, \varepsilon}(x):=\lambda_{r, \varepsilon}|x|^{1 / 2} I(|x|<\sqrt{d})+(1+\varepsilon r|x|)^{-d} I(|x| \geq \sqrt{d}) .
$$

In view of the following Lemma 7.9 this concludes the proof of the bound (7.30). 
If the region $\Omega$ is not admissible, then we change variables to $w=r^{-1} v$ split the left-hand side of (7.30) into integrals over unit cells $E:=\left[-\frac{1}{2}, \frac{1}{2}\right)^{d}$ in order to find

$$
I_{\zeta}=\sum_{m \in \mathbb{Z}^{d} \backslash\{0\}} I_{\zeta}(m), \text { where } I_{\zeta}(m) \stackrel{\text { def }}{=} r^{d} \int_{E} \frac{\mid \widehat{\zeta}_{\varepsilon}(r(m+w) \mid}{\left(q^{1 / 2} r^{-1}+\|w\|_{\infty}\right)^{d / 2}} \mathrm{~d} w .
$$

Because of $\sum_{j=1}^{d}\left|u_{j}\right|^{1 / 2} \geq\|u\|^{1 / 2}$ we can further estimate (7.37) by

$$
\left|\widehat{k}_{B, \varepsilon}\left(Z^{-1} u\right)\right| \leq \exp \left\{-\|\varepsilon u\|^{1 / 2}\right\}
$$

Recalling the definition (7.34) and the estimates (7.35)-(7.36) for $u=Z w$ shows that

$\left|\widehat{\zeta}_{\varepsilon}(r w)\right| \ll_{k} d_{Q} \varepsilon^{-k+1}(r\|Z w\|+1)^{-k} \ll d_{Q} \varepsilon^{-k+1}\left(q c_{B}\right)^{k / 2}(r\|w\|+1)^{-k}$.

Thus, taking $k=d+1$ we find

$$
I_{\zeta} \ll_{d} d_{Q} q^{d / 2} c_{B}^{(d+1) / 2} \varepsilon^{-d} .
$$

The last remark easily follows by comparing the volume of the bodies $\{\|B x\| \leq 1\}$ and $\left\{\left\|Q_{+}^{1 / 2} x\right\| \leq 1\right\}$ : Using (7.1) leads to $|\operatorname{det} Q|^{1 / 2} \leq|\operatorname{det} B| \leq$ $\left(c_{B}\right)^{d / 2}|\operatorname{det} Q|^{1 / 2}$.

Lemma 7.9 For an admissible lattice $\Gamma$ we have for any weight function $\omega(x)>0$ on $\mathbb{R}$, such that $\omega_{\infty}:=1+\max _{x} \omega(x)(1+|x|)^{p}<\infty$, where $p \in \mathbb{N}$ and $\varepsilon>0$, the bound

$$
S_{\Gamma, \varepsilon} \stackrel{\text { def }}{=} \sum_{\left(\gamma_{1}, \ldots, \gamma_{d}\right) \in \Gamma \backslash\{0\}}\left|\frac{\omega_{r, \varepsilon}\left(\gamma_{1}\right) \ldots \omega_{r, \varepsilon}\left(\gamma_{d}\right)}{\gamma_{1} \ldots \gamma_{d}}\right| \ll_{d} \omega_{\infty} \lambda_{r, \varepsilon}^{d-1} \frac{\bar{\lambda}_{r, \varepsilon, \Gamma}}{\operatorname{Nm}(\Gamma)}
$$

where $\omega_{r, \varepsilon}(x):=\lambda_{r, \varepsilon}|x|^{\frac{1}{2}} I(|x|<\sqrt{d})+\omega(\varepsilon r x) I(|x| \geq \sqrt{d})$ and $\lambda_{r, \varepsilon}, \bar{\lambda}_{r, \varepsilon, \Gamma}$ are as introduced in Lemma 7.8.

Proof First, we make a decomposition of $\Gamma$ as follows. For any $\left(x_{1}, \ldots, x_{d}\right) \in$ $\mathbb{R}^{d}$ with $\left|x_{1} \cdots x_{d}\right| \geq \operatorname{Nm}(\Gamma)$ let $m_{j} \in \mathbb{Z}$ be the unique integers satisfying $2>$ $\left|2^{m_{j}} x_{j}\right| d^{-1 / 2} \geq 1$ for $j=2, \ldots, d$. We have $\left|x_{1}\right| \geq \operatorname{Nm}(x)\left|x_{2} \ldots x_{d}\right|^{-1} \geq$ $\mathrm{Nm}(\Gamma) d^{(1-d) / 2} \prod_{j=2}^{d} 2^{m_{j}-1}$ and this implies that $\left|2^{m_{1}} x_{1}\right| \in\left[k c_{\Gamma},(k+1) c_{\Gamma}\right)$ for a unique integer $k \geq 1$, where $m_{1} \in \mathbb{Z}$ is determined by $m_{1}+m_{2}+\ldots+$ $m_{d}=0$ and $c_{\Gamma}=d^{(1-d) / 2} 2^{-d+1} \mathrm{Nm}(\Gamma)$. Introducing the lattice

$$
E_{d}:=\left\{m=\left(m_{1}, \ldots, m_{d}\right) \in \mathbb{Z}^{d}: m_{1}+\ldots+m_{d}=0\right\} \subset \mathbb{Z}^{d}
$$


and the interval $B_{k}:=\left[k c_{\Gamma},(k+1) c_{\Gamma}\right)$, we can write

$$
I\left(\left|x_{1} \ldots x_{d}\right| \geq \operatorname{Nm}(\Gamma)\right)=\sum_{m \in E_{d}} \sum_{k \in \mathbb{N}} I_{B_{k}}\left(\left|2^{m_{1}} x_{1}\right|\right) \prod_{j=2}^{d} I_{[\sqrt{d}, 2 \sqrt{d})}\left(\left|2^{m_{j}} x_{j}\right|\right),
$$

and hence

$$
S_{\Gamma, \varepsilon}=\sum_{m \in E_{d}} \sum_{k \in \mathbb{N}} \sum_{\gamma \in \Gamma} I_{B_{k}}\left(\left|2^{m_{1}} \gamma_{1}\right|\right) \prod_{j=2}^{d} I_{[\sqrt{d}, 2 \sqrt{d})}\left(\left|2^{m_{j}} \gamma_{j}\right|\right)\left|\frac{\omega_{r, \varepsilon}\left(\gamma_{1}\right) \ldots \omega_{r, \varepsilon}\left(\gamma_{d}\right)}{\gamma_{1} \ldots \gamma_{d}}\right| .
$$

We also introduce the obvious notations $\operatorname{Nm}(x):=\left|x_{1} \cdots x_{d}\right|, 2^{m} x=$ $\left(2^{m_{1}} x_{1}, \ldots 2^{m_{d}} x_{d}\right), m \in E_{d}$ and $2^{m} \Gamma$ for the rescaled lattice $\left\{2^{m} \gamma: \gamma \in \Gamma\right\}$. Note that $\operatorname{Nm}\left(2^{m} \gamma\right)=\operatorname{Nm}(\gamma)$ and hence $\operatorname{Nm}(\Gamma)=\operatorname{Nm}\left(2^{m} \Gamma\right)$. Defining $C_{k}:=B_{k} \times[\sqrt{d}, 2 \sqrt{d})^{d-1}$ and $h(x):=(1+|x|)^{-p}$ (where $p \in \mathbb{N}$ is the same as in the assumptions of the lemma), we may rewrite and bound (7.41) by

$$
\begin{aligned}
S_{\Gamma, \varepsilon} & =\sum_{m \in E_{d}}\left(\sum_{k \in \mathbb{N}} \sum_{\eta \in 2^{m} \Gamma} I_{C_{k}}(\eta) \prod_{j=1}^{d} \frac{\omega_{r, \varepsilon}\left(2^{-m_{j}} \eta_{j}\right)}{\left|\eta_{j}\right|}\right) \\
& \ll_{d} \omega_{\infty} \sum_{m \in E_{d}} \sum_{k \in \mathbb{N}}\left(\left(\sum_{\eta \in 2^{m} \Gamma} I_{C_{k}}(\eta)\right) \frac{h_{r, \varepsilon}\left(c_{\Gamma} 2^{-m_{1}} k\right)}{c_{\Gamma} k}\right) \prod_{j=2}^{d} h_{r, \varepsilon}\left(2^{-m_{j}}\right),
\end{aligned}
$$

where $h_{r, \varepsilon}(x):=\lambda_{r, \varepsilon}|x|^{\frac{1}{2}} I(|x|<1)+h(\varepsilon r x) I(|x| \geq 1)$. In order to perform the summation in $k$ and $\eta$ in (7.42) we first observe that

$$
\sum_{\eta \in 2^{m} \Gamma} I_{C_{k}}(\eta) \leq 1
$$

Proof of (7.43): Assume that two different lattice points $\eta, \eta^{\prime} \in 2^{m} \Gamma$ lie in $C_{k}$. Then we have $\left|\eta_{1}-\eta_{1}^{\prime}\right|<c_{\Gamma}$ and $\max _{2 \leq j \leq d}\left|\eta_{j}-\eta_{j}^{\prime}\right|<\sqrt{d}$. Since $\eta-\eta^{\prime} \in$ $2^{m} \Gamma \backslash\{0\}$ implies $\left|\eta_{2}-\eta_{2}^{\prime}\right| \cdots\left|\eta_{d}-\eta_{d}^{\prime}\right| \geq(\mathrm{Nm} \Gamma) / c_{\Gamma}=d^{(d-1) / 2} 2^{(d-1)}$ and hence $\left|\left(\eta_{2}-\eta_{2}^{\prime}\right)\right| \geq 2 \sqrt{d}$ for some $j \geq 2$, we get at a contradiction which proves (7.43).

Estimating the following sum in $k$ by an integral, we obtain

$$
\sum_{k=1}^{\infty} \frac{h_{r, \varepsilon}(\alpha k)}{k} \ll \lambda_{r, \varepsilon} I(\alpha<1)+\log \left(1+\frac{2}{\alpha r \varepsilon}\right) \stackrel{\text { def }}{=} \bar{h}_{r, \varepsilon}(\alpha)
$$


Hence, making use of (7.43) and (7.44) in (7.42), shows that

$$
S_{\Gamma, \varepsilon} \ll_{d} \omega_{\infty}\left(c_{\Gamma}\right)^{-1} \sum_{m \in E_{d}} H\left(2^{-m}\right),
$$

where $2^{m}:=\left(2^{m_{1}}, \ldots, 2^{m_{d}}\right)$ and $H(x):=\bar{h}_{r, \varepsilon}\left(c_{\Gamma} x_{1}\right) h_{r, \varepsilon}\left(x_{2}\right) \cdots h_{r, \varepsilon}\left(x_{d}\right)$.

Let $E_{d}^{\prime}$ denote the subset of $E_{d}$ consisting of all lattice points $\left(m_{1}, \ldots, m_{d}\right)$ $\in E_{d}$ with $m_{1} \leq 0$. We claim that

$$
\sum_{m \in E_{d}^{\prime}} H\left(2^{-m}\right) \ll_{d}\left(\lambda_{r, \varepsilon}+\log \left(1+\frac{1}{\operatorname{Nm}(\Gamma) r \varepsilon}\right)\right) \lambda_{r, \varepsilon}^{d-1} .
$$

Proof of (7.46) Let $m \in E_{d}^{\prime} \backslash\{0\}$. Assume for definiteness that $m_{1}, \ldots, m_{l-1} \leq$ 0 and $m_{l}, \ldots, m_{d}>0$. By definition of $E_{d}$ we get $2 \sum_{j=l}^{m} m_{j}=\sum_{j=1}^{d}\left|m_{j}\right| \geq$ $\|m\|_{2}$. Since $h_{r, \varepsilon}\left(2^{-k}\right) \leq 1$ for $k \leq 0$ and otherwise $h_{r, \varepsilon}\left(2^{-k}\right)=\lambda_{r, \varepsilon} 2^{-k / 2}$, we obtain

$$
\begin{aligned}
H\left(2^{-m}\right) & \ll_{d}\left(\lambda_{r, \varepsilon}+\log \left(1+\frac{1}{\operatorname{Nm}(\Gamma) r \varepsilon}\right)\right) \lambda_{r, \varepsilon}^{d-l} \prod_{j=l}^{d} 2^{-m_{j} / 2} \\
& \ll_{d}\left(\lambda_{r, \varepsilon}+\log \left(1+\frac{1}{\operatorname{Nm}(\Gamma) r \varepsilon}\right)\right) \lambda_{r, \varepsilon}^{d-l} 2^{-\|m\| / 4} .
\end{aligned}
$$

Thus, splitting the sum according to the number of positive coordinates and then summing over the $(d-1)$-dimensional lattice $E_{d}$ yields (7.46).

In order to bound the sum over the complement of $E_{d}^{\prime}$, we again split the sum according to the number of positive coordinates. For simplicity, we may assume that $m_{1}, m_{2}, \ldots, m_{l}>0$ and $m_{l+1}, \ldots, m_{d} \leq 0$. Similar to the previous case, we find that

$$
H\left(2^{-m}\right) \ll_{d}\left(\|m\|+\lambda_{r, \varepsilon}+\log \left(1+\frac{1}{\operatorname{Nm}(\Gamma) r \varepsilon}\right)\right) \lambda_{r, \varepsilon}^{l-1}\left(\prod_{j=2^{-}}^{l} 2^{-\frac{m_{j}}{2}}\right) \min \left(1,(r \varepsilon)^{-d p} 2^{-p\|m\| / 2}\right) .
$$

If we parameterize the $(d-1)$-dimensional lattice $E_{d}$ by $\left(m_{1}, \bar{m}\right)$, where $m_{1}=-\left(m_{2}+\ldots+m_{d}\right)$ and $\bar{m}=\left(m_{2}, \ldots, m_{d}\right) \in \mathbb{Z}^{d-1}$, and split the summation into a ball of radius $\|\bar{m}\|_{2} \leq R_{\varepsilon}:=3 d \log \left(2+(r \varepsilon)^{-1}\right)$ and its complement, where $(r \varepsilon)^{-d p} 2^{-p\|m\|_{2} / 2} \leq(r \varepsilon)^{-d p} 2^{-p\|\bar{m}\|_{2} / 2} \leq 1$, we can bound the sum corresponding to a fixed $l$ by

$$
\begin{aligned}
& \ll_{d} \lambda_{r, \varepsilon}^{l-1}\left(\sum_{\|\bar{m}\|_{2} \leq R_{\varepsilon}}\left(\bar{\lambda}_{r, \varepsilon, \Gamma}+\|\bar{m}\|\right) \prod_{j=2}^{l} 2^{-m_{j} / 2}+\sum_{\|\bar{m}\|_{2}>R_{\varepsilon}}\left(\bar{\lambda}_{r, \varepsilon, \Gamma}+\|\bar{m}\|\right)(r \varepsilon)^{-d p} 2^{-p\|\bar{m}\|_{2} / 2}\right) \\
& \ll_{d} \lambda_{r, \varepsilon}^{l-1}\left(\bar{\lambda}_{r, \varepsilon, \Gamma} \log \left(2+\frac{1}{r \varepsilon}\right)^{d-1-(l-1)}+\bar{\lambda}_{r, \varepsilon, \Gamma}\right) \ll_{d} \lambda_{r, \varepsilon}^{d-1} \bar{\lambda}_{r, \varepsilon, \Gamma},
\end{aligned}
$$

where we have estimated the sums by comparison with the corresponding integrals. Using this estimate for each $l=1, \ldots, d-1$ together with (7.46) in (7.45) yields the bound (7.40). 


\subsection{Applications of Theorem 2.2}

We start by smoothing the indicator function of the region $\Omega$. We choose weights $\mathrm{v}=\mathrm{v}_{ \pm \varepsilon}$ as defined in (7.3) with $\varepsilon \in\left(0, \varepsilon_{0}\right]$ and the related $\zeta=\zeta_{\varepsilon}$, see Sect. 7.2, corresponding to parallelepipeds $\Omega=B^{-1}[-1,1]^{d}$ satisfying $Q_{+} \leq B^{T} B \leq c_{B} Q_{+}$, compare (7.1). Recalling (7.6), where we have used Lemma 7.1 to estimate the $\varepsilon$-smoothing error, yields a total error

$$
\Delta_{r} \stackrel{\text { def }}{=}\left|\operatorname{vol}_{\mathbb{Z}}\left(E_{a, b} \cap r \Omega\right)-\operatorname{vol}\left(E_{a, b} \cap r \Omega\right)\right| \ll_{d} d_{Q}(b-a) \varepsilon r^{d-2}+\max _{ \pm}\left|R\left(I_{E_{a, b}} \mathrm{v}_{ \pm \varepsilon, r}\right)\right| .
$$

Now we can apply Theorem 2.2 in order to bound the latter remainder $\left|R\left(I_{E_{a, b}} \mathrm{v}_{ \pm \varepsilon, r}\right)\right|$ as follows. In (6.14) we shall estimate $\left\|\widehat{\zeta}_{\varepsilon}\right\|_{*, r}$ by using $\left\|\mathrm{v}_{\varepsilon}\right\|_{Q} \ll_{d} d_{Q}$ of Lemma 7.1, $\left\|\widehat{\zeta}_{\varepsilon}\right\|_{1} \ll_{d}\left(\log \varepsilon^{-1}\right)^{d}$ of Lemma 7.2 and

$$
\left\|\widehat{\zeta}_{\varepsilon}\right\|_{*, r} \ll_{d} q^{d / 4}\left(\left(\frac{q}{q_{0}}\right)^{d / 2} \log \left(\varepsilon^{-1}\right)^{d}+q_{0}^{-d / 4} c_{B}^{d / 2} \lambda_{r, \varepsilon}^{d-1} \frac{\bar{\lambda}_{r, \varepsilon, \Gamma}}{\operatorname{Nm}(\Gamma)}\right)
$$

of Lemma 7.8 for admissible regions $\Omega$, i.e. (7.29) holds, to get

$$
\begin{aligned}
\Delta_{r} \ll \beta, d & d_{Q} r^{d-2}\left(\varepsilon(b-a)+w+a_{Q}\left(\log \frac{1}{\varepsilon}\right)^{d} \rho_{Q, b-a}^{w}(r)\right) \\
& +d_{Q} q^{d / 4} r^{d / 2}\left(\left(\frac{q}{q_{0}}\right)^{d / 2} \log \left(\varepsilon^{-1}\right)^{d}+q_{0}^{-d / 4} c_{B}^{d / 2} \lambda_{r, \varepsilon}^{d-1} \frac{\bar{\lambda}_{r, \varepsilon, \Gamma}}{\operatorname{Nm}(\Gamma)}\right) \log \left(1+\frac{b-a}{q_{0}^{1 / 2} r}\right),
\end{aligned}
$$

where $a_{Q}:=q c_{Q}=q|\operatorname{det} Q|^{1 / 4-\beta / 2}=C_{Q}\left(d_{Q}\right)^{-1}$, provided that $0<w<$ $(b-a) / 4$. This bound holds for admissible parallelepipeds $\Omega$ only. If $\Omega$ is not admissible, then we have to replace the smoothing error (7.48) by

$$
\left\|\widehat{\zeta}_{\varepsilon}\right\|_{*, r} \ll_{d} q^{d / 4}\left(\left(q / q_{0}\right)^{d / 2} \log \left(\varepsilon^{-1}\right)^{d}+d_{Q} q^{d / 2}\left(c_{B}\right)^{(d+1) / 2} \varepsilon^{-d}\right),
$$

that is (7.31) of Lemma 7.8. With these bounds we are ready to prove the main statements on the lattice point remainder for hyperbolic shells.

Proof of Corollary 2.5 For wide shells, i.e. $b-a>q$, we optimize (7.49) in the smoothing parameter $w$ first by choosing $w=\mathrm{W}\left(q T_{+} / 2\right)^{2} / T_{+}$, where $\mathrm{W}$ denotes the upper branch, defined on the interval $\left(-\mathrm{e}^{-1}, \infty\right)$, of the inverse function of $x \mapsto x e^{x}$. (The function $W$ is also known as the Lambert- $W$ function, see [15] for more details and some applications.)

Since $x \mapsto W(x)^{2} / x$ has a global maximum at $x=\mathrm{e}$ with value $\mathrm{e}^{-1}$, we find $w \leq q /(2 \mathrm{e})<(b-a) / 4$ as required in the restrictions (6.6). This leads to the partial bound

$$
d_{Q} w+C_{Q} c_{Q}^{-1}\left(T_{+} w\right)^{-1 / 2} \mathrm{e}^{-\left(T_{+} w\right)^{1 / 2}} \ll d_{Q} \frac{\mathrm{W}\left(q T_{+} / 2\right)^{2}}{T_{+}} \ll d_{Q} \frac{\log \left(q T_{+}+1\right)^{2}}{T_{+}},
$$


where we used that $W(x) \leq \log (x+1)$ and $W(x)^{-1} \exp (-W(x))=$ $x^{-1}$. Next, we calibrate the $\varepsilon$-dependent terms in (7.49) by choosing $\varepsilon=$ $T_{-}^{\frac{d}{2}-2-\delta}(b-a)^{-1} / 15$. Again, this choice satisfies the required restrictions, i.e. $\varepsilon \leq \varepsilon_{0}=1 / 15$. Because of

$$
\begin{aligned}
& \varepsilon(b-a) \leq a_{Q}(b-a)_{q} c_{Q} T_{-}^{\frac{d}{2}-2-\delta}, \quad \log \varepsilon^{-1} \ll \log (r+1) \quad \text { and } \\
& \frac{\bar{\lambda}_{r, \varepsilon, \Gamma}}{\log (r+1)} \ll \max \left\{1, \frac{\log \left(2+\frac{r^{d+1}}{\operatorname{Nm}(\Gamma)}\right)}{\log (r+1)}\right\} \ll_{d} \log \left(2+\frac{1}{\operatorname{Nm}(\Gamma)}\right),
\end{aligned}
$$

compare the definition in Lemma 7.8, we can simplify (7.49) to

$$
\begin{aligned}
\Delta_{r} \ll \beta, d & d_{Q} r^{d-2} \rho_{Q, b-a}^{\mathrm{hyp}+}(r)+d_{Q} q^{\frac{d}{4}} r^{\frac{d}{2}} \log (r+1)^{d} \\
& \times\left(\left(\frac{q}{q_{0}}\right)^{\frac{d}{2}}+\frac{c_{B}^{d / 2} q_{0}^{-d / 4}}{\operatorname{Nm}(\Gamma)} \log \left(2+\frac{1}{\operatorname{Nm}(\Gamma)}\right)\right) \log \left(1+\frac{b-a}{q_{0}^{1 / 2} r}\right),
\end{aligned}
$$

where

$$
\begin{array}{r}
\rho_{Q, b-a}^{\text {hyp }+}(r) \stackrel{\text { def }}{=} \inf _{T_{+}, T_{-}}^{*}\left\{\operatorname { l o g } ( ( b - a ) T _ { - } ^ { - ( \frac { d } { 2 } - 2 - \delta ) } + 1 ) ^ { d } \left(a_{Q} q^{(2 \beta d-1) / 2}\left(c_{Q} T_{-}^{\frac{d}{2}-2-\delta}+\gamma_{\left[T_{-}, 1\right], \beta}(r)\right)\right.\right. \\
\left.\left.+a_{Q} \gamma_{\left(1, T_{+}\right], \beta}(r) \log \left(T_{+}+1\right)+\frac{\log \left(q T_{+}+1\right)^{2}}{T_{+}}\right)\right\}
\end{array}
$$

and the infimum is taken over all $T_{-} \in\left[q_{0}^{-1 / 2} r^{-1}, 1\right], T_{+} \geq 1$. This proves the first part of Corollary 2.5. Next, we consider the case of thin shells, i.e. $b-a \leq q$. Here we take $\varepsilon=T_{-}^{\frac{d}{2}-2-\delta} / 15$ and $w=T_{-}^{\frac{d}{2}-2-\delta}(b-a) / 4$ in (7.49), noting that $d_{Q}(w+\varepsilon(b-a)) \leq a_{Q}(b-a) c_{Q} T_{-}^{\frac{d}{2}-2-\delta}$, in order to get the bound (7.51), whereby the factor $\rho_{Q, b-a}^{\text {hyp }}(r)$, depending on the Diophantine properties of $Q$, has to be replaced by

$$
\begin{aligned}
\rho_{Q, b-a}^{\text {hyp }}(r) \stackrel{\text { def }}{=} \inf _{T_{-}, T_{+}}^{*}\left\{a_{Q} \log \left(1+T_{-}^{-\left(\frac{d-4}{2}-\delta\right)}\right)^{d}\right. & (b-a)\left(c_{Q} T_{-}^{\frac{d-4}{2}-\delta}+\gamma_{\left[T_{-}, 1\right], \beta}(r)\right) \\
& \left.\left.+\gamma_{\left(1, T_{+}\right], \beta}(r)\left(\log \left((b-a)^{*} T_{+}\right)+1\right)\right)\right\} .
\end{aligned}
$$

In the last equation the infimum is taken over all $T_{-} \in\left[q_{0}^{-1 / 2} r^{-1}, 1\right]$ and $T_{+} \geq 1$ with

$$
T_{+} \geq 4(b-a)^{-1} T_{-}^{-\left(\frac{d}{2}-2-\delta\right)} \max \left\{1, \log \left(c_{Q}^{2}(b-a) T_{-}^{\frac{d}{2}-2-\delta}\right)^{2}\right\},
$$

where the last condition ensures that

$$
c_{Q}^{-1}\left(T_{+} w\right)^{-1 / 2} \mathrm{e}^{-\left(T_{+} w\right)^{1 / 2}} \leq c_{Q}(b-a) T_{-}^{\frac{d}{2}-2-\delta} .
$$


Finally, we note that Corollary 4.11 implies that $\gamma_{\left[T_{-}, 1\right], \beta}(r) \rightarrow 0$ and also $\gamma_{\left[1, T_{+}\right], \beta}(r) \rightarrow 0$ for $r \rightarrow \infty$ and any fixed $T_{-} \in\left[q_{0}^{-1 / 2} r^{-1}, 1\right], T_{+} \geq 1$, when $Q$ is irrational. Thus, we conclude that $\rho_{Q, b-a}^{\text {hyp }+}(r) \rightarrow 0$, resp. $\rho_{Q, b-a}^{\text {hyp }-}(r) \rightarrow 0$, for $r \rightarrow \infty$ and fixed $b-a$.

Corollary 7.10 Consider an indefinite quadratic form $Q$ in $d \geq 5$ variables and a (not necessary admissible) parallelepiped $\Omega$ satisfying (7.1) and $\max \{|a|,|b|\} \leq c_{0} r^{2}$, where $c_{0}>0$ is chosen as in Lemma 7.1. Then for all $b-a \leq 1$

$$
\Delta_{r} \ll_{\beta, d} d_{Q} r^{d-2}\left(\rho_{Q, b-a}^{\mathrm{hyp} *}(r)+(b-a) r^{1-d / 2} q^{(d-2) / 4} \log (1+r)^{d}\left(q / q_{0}\right)^{(d+1) / 2}\left(c_{B}\right)^{(d+1) / 2}\right),
$$

where $\rho_{Q, b-a}^{\text {hyp* }}$ is defined in (7.53). In particular, for irrational $Q$ we have $\rho_{Q, b-a}^{\text {hyp* }}(r) \rightarrow 0$ for $r \rightarrow \infty$, provided that $b-a$ is fixed.

Proof. We shall argue similar as in the previous proof of Corollary 2.5, but here we can only use (7.50) to bound $\left\|\widehat{\zeta}_{\varepsilon}\right\|_{*, r}$, since $\Omega$ is not necessarily admissible. Thus, we have to replace the error bound (7.49) for the lattice remainder by

$$
\begin{aligned}
\Delta_{r} \ll_{\beta, d} d_{Q} r^{d-2}\left(\varepsilon(b-a)+w+a_{Q}\left(\log \frac{1}{\varepsilon}\right)^{d} \rho_{Q, b-a}^{w}(r)\right)+d_{Q} q^{d / 4} r^{d / 2} \\
\quad \times\left(\left(\frac{q}{q_{0}}\right)^{d / 2}\left(\log \frac{1}{\varepsilon}\right)^{d}+d_{Q} q^{d / 2}\left(c_{B}\right)^{(d+1) / 2} \varepsilon^{-d}\right) \log \left(1+\frac{b-a}{q_{0}^{1 / 2} r}\right) .
\end{aligned}
$$

Now the right-hand side can be optimized by taking

$$
\varepsilon=\left(15 \log \left(1+T_{-}^{-\left(\frac{d}{2}-2-\delta\right)}\right)\right)^{-1} \quad \text { and } \quad w=T_{-}^{\frac{d}{2}-2-\delta}(b-a) / 4
$$

and this leads to the bound

$$
\begin{aligned}
\Delta_{r} \ll \beta, d & d_{Q} r^{d-2} \rho_{Q, b-a}^{\text {hyp }}(r)+d_{Q} q^{d / 4} r^{d / 2}( \\
& \log (1+r)^{d}\left(q / q_{0}\right)^{d / 2} \\
& \left.+d_{Q} q^{d / 2}\left(c_{B}\right)^{(d+1) / 2} \log (1+r)^{d}\right) \log \left(1+\frac{|b-a|}{q_{0}^{1 / 2} r}\right),
\end{aligned}
$$

where

$$
\begin{aligned}
\rho_{Q, b-a}^{\text {hyp } *}(r) \stackrel{\text { def }}{=} \inf \left\{a _ { Q } \operatorname { l o g } ( 1 + T _ { - } ^ { - ( \frac { d } { 2 } - 2 - \delta ) } ) ^ { d } \left((b-a)\left(c_{Q} T_{-}^{\left(\frac{d}{2}-2-\delta\right)}+\gamma_{\left[T_{-}, 1\right], \beta}(r)\right)\right.\right. \\
\left.\left.+\gamma_{\left(1, T_{+}\right], \beta}(r) \log \left((b-a) T_{+}\right)\right)+\frac{b-a}{\log \left(1+T_{-}^{-\left(\frac{d}{2}-2-\delta\right)}\right)}\right\}
\end{aligned}
$$

and the infimum is taken over all $T_{-} \in\left[q_{0}^{-1 / 2} r^{-1}, 1\right]$ and

$$
T_{+} \geq 4(b-a)^{-1} T_{-}^{-\left(\frac{d}{2}-2-\delta\right)} \max \left\{1, \log \left(c_{Q}^{2}(b-a) T_{-}^{\frac{d}{2}-2-\delta}\right)^{2}\right\} .
$$


The next corollary provides a lower bound for the number of lattice points and is useful for proving quantitative bounds in the Oppenheim conjecture.

Corollary 7.11 For the special choice $B=Q_{+}^{1 / 2}$, i.e. $\Omega=Q_{+}^{-1 / 2}[-1,1]^{d}$ and $c_{B}=1$, and all $\max \{|a|,|b|\} \leq r^{2} / 5$ and $b-a \leq 1$ there exists constants $b_{\beta, d}>0$ and $\tilde{b}_{\beta, d}>0$, depending on $\beta$ and $d$ only, such that for all $r \geq$ $\tilde{b}_{\beta, d} q^{1 / 2}\left(q / q_{0}\right)^{(d+1) /(d-2)}$

$$
\Delta_{r} \leq \frac{\operatorname{vol} H_{r}}{5}+b_{\beta, d} d_{Q} r^{d-2} \rho_{Q, b-a}^{\text {hyp } * *}(r)
$$

where $c_{Q}=|\operatorname{det} Q|^{1 / 4-\beta / 2}, a_{Q}=q c_{Q}$ and

$$
\begin{aligned}
\rho_{Q, b-a}^{\text {hyp** }}(r) \stackrel{\text { def }}{=} \inf \{ & a_{Q}\left(( b - a ) \left(c_{Q} T_{-}^{\frac{d}{2}-2-\delta}\right.\right. \\
& \left.\left.\left.+\gamma_{\left[T_{-}, 1\right], \beta}(r)\right)+\gamma_{\left(1, T_{+}\right], \beta}(r) \log \left((b-a) T_{+}\right)\right)\right\}
\end{aligned}
$$

and the infimum is taken over all $T_{-} \in\left[q_{0}^{-1 / 2} r^{-1}, 1\right]$ and $T_{+} \geq 1$ with

$$
T_{+} \geq C_{\beta, d} \frac{1}{(b-a)} \max \left\{\log \left(\frac{b-a}{q c_{\beta, d}}\right)^{2}, 1\right\}
$$

and $C_{\beta, d}, c_{\beta, d} \geq 1$ are constants depending on $d$ and $\beta$ only.

Proof Here we only consider the special region $\Omega=Q_{+}^{-1 / 2}[-1,1]^{d}$, i.e. $B=Q_{+}^{1 / 2}$ and thus (7.1) is valid with $c_{B}=1$. Since $\Omega$ is not necessarily admissible, we have to argue as in the previous proof (of Corollary 7.10): Starting with the estimate (7.52), we can take $\varepsilon=\left(30 a_{d} b_{\beta, d}\right)^{-1}$ and $w=$ $(b-a) \varepsilon$ in the optimization procedure, where $a_{d} \geq 1$, resp. $b_{\beta, d} \geq 1$, denotes the implicit constant in (7.12) (see Lemma 7.1), resp. (7.52). (Of course, we have $\varepsilon \in\left(0, \varepsilon_{0}\right]$ and $0<w<(b-a) / 4$ as required.) This yields

$$
\Delta_{r} \leq \frac{\operatorname{vol} H_{r}}{15}+b_{\beta, d} d_{Q} r^{d-2} a_{Q}\left(\log \frac{1}{\varepsilon}\right)^{d} \rho_{Q, b-a}^{w}(r)+\bar{b}_{\beta, d}(b-a) d_{Q} q^{\frac{d-2}{4}} r^{d / 2-1}\left(\frac{q}{q_{0}}\right)^{\frac{d+1}{2}},
$$

where $\bar{b}_{\beta, d}:=b_{\beta, d}\left(\varepsilon^{-d}+\log \left(\varepsilon^{-1}\right)^{d}\right)$ depends on $\beta$ and $d$ only. Again referring to Lemma 7.1, we also see that

$$
\bar{b}_{\beta, d}(b-a) d_{Q} q^{(d-2) / 4} r^{d / 2-1}\left(\frac{q}{q_{0}}\right)^{(d+1) / 2} \leq \frac{\operatorname{vol} H_{r}}{15}
$$

if we choose $r \geq \tilde{b}_{\beta, d} q^{1 / 2}\left(q / q_{0}\right)^{(d+1) /(d-2)}$ with $\tilde{b}_{\beta, d}=\left(15 a_{d} \bar{b}_{\beta, d}\right)^{-1}$. Finally, we make the restriction $T_{+} \geq w^{-1} \max \left\{\log \left(\left(15 a_{d} \bar{b}_{\beta, d}\right)^{-1} q^{-1}(b-\right.\right.$ 
a) $\left.)^{2}, 1\right\}$ to ensure that

$$
b_{\beta, d}\left(\log \varepsilon^{-1}\right)^{d} q r^{d-2} d_{Q}\left(T_{+} w\right)^{-1 / 2} \exp \left(-\left|T_{+} w\right|^{1 / 2}\right) \leq \operatorname{vol} H_{r} / 15 .
$$

Collecting the remaining terms proves (7.54).

Now we consider elliptic shells as well and optimize the lattice remainder as in the case of 'wide shells'. In contrast to the previous cases, the error caused by the smoothing of the region $\Omega$ is not present here.

Proof of Corollary 2.4 In the case of ellipsoids, i.e. $Q$ is a positive definite form, we choose the (not necessary admissible) parallelepiped $\Omega:=$ $B^{-1}[-1,1]^{d}$ with $B=Q_{+}^{1 / 2}$ and $r=\sqrt{2 b} \geq q^{1 / 2}$, resp. $2 b=r^{2}, a=0$ and $\varepsilon=1 / 15$. Then (7.1) is satisfied with $c_{B}=1$ and $E_{0, b} \subset r \Omega$, i.e. $H_{r}:=E_{a, b} \cap r \Omega=E_{a, b}$. Moreover, since $E_{0, b}$ does not intersect $r(\partial \Omega)_{2 \varepsilon}$ (the $2 \varepsilon r$-boundary of $r \Omega$ as defined in (7.3)), we get an error $R_{\varepsilon, r}=0$ for smoothing the indicator function of $r \Omega$. Hence, we may remove the term proportional to $(b-a) \varepsilon$ in (7.47). Note that apart from Lemma 7.1 the indefiniteness of $Q$ has not been used in all arguments so far. In contrast to the case of hyperbolic shells, we optimize (6.14) in $w$ first. Again including the bound $\left\|\mathrm{v}_{\varepsilon}\right\|_{Q} \ll_{d} d_{Q}$ of Lemma 7.1 and here taking $w=\mathrm{W}\left(q T_{+} / 4\right)^{2} / T_{+}$, where $\mathrm{W}$ denotes the upper branch of the Lambert- $W$-function (for more details on the Lambert- $W$-function see the proof of Corollary 2.5 on p. 71), and noting that $w \leq q /(4 e)<(b-a) / 4$, leads (as in the proof of Corollary 2.5) to the bound

$$
\begin{aligned}
\Delta_{r} \ll \beta, d & r^{d-2}\left(C_{Q}\left(q^{(2 \beta d-1) / 2}\left(c_{Q} T_{-}^{\frac{d}{2}-2-\delta}+\gamma_{\left[T_{-}, 1\right], \beta}(r)\right)+\gamma_{\left(1, T_{+}\right] \beta}(r) \log \left(T_{+}+1\right)\right)\right. \\
& \left.+d_{Q} \frac{\log \left(1+q T_{+}\right)^{2}}{T_{+}}\right)+d_{Q} q^{d / 4} r^{d / 2}\left(\left(q / q_{0}\right)^{d / 2}+d_{Q} q^{d / 2}\right) \log \left(1+\frac{r}{q_{0}^{1 / 2}}\right),
\end{aligned}
$$

where $T_{-} \in\left[q_{0}^{-1 / 2} r^{-1}, 1\right]$ and $T_{+} \geq 1$. This can be rewritten as

$$
\Delta_{r} \ll_{\beta, d} d_{Q} r^{d-2} \rho_{Q}(r)+d_{Q} q^{d / 4} r^{d / 2}\left(q / q_{0}\right)^{d / 2} \log \left(1+r / q_{0}^{1 / 2}\right)
$$

with

$$
\rho_{Q}^{\text {ell }}(r) \stackrel{\text { def }}{=} \inf \left\{a_{Q}\left(q^{\beta d-\frac{1}{2}}\left(c_{Q} T_{-}^{\frac{d}{2}-2-\delta}+\gamma_{\left[T_{-}, 1\right], \beta}(r)\right)+\gamma_{\left(1, T_{+}\right], \beta}(r) \log \left(T_{+}+1\right)\right)+\frac{\log \left(1+q T_{+}\right)^{2}}{T_{+}}\right\},
$$

where the infimum is taken over all $T_{-} \in\left[q_{0}^{-1 / 2} r^{-1}, 1\right]$ and $T_{+} \geq 1$. Note that as in the indefinite case $\lim _{r \rightarrow \infty} \rho_{Q}^{\text {ell }}(r)=0$ if $Q$ is irrational by Corollary 4.11. This proves Corollary 2.4. Furthermore, we remark that $\operatorname{vol} H_{r}=\operatorname{vol}(r \Omega \cap$ $\left.E_{0, b}\right)=d_{Q} \omega_{d} r^{d}$, where $\omega_{d}$ denotes the volume of the unit $d$-ball. 
Similar arguments can be used in order to obtain related bounds for both wide $(b-a>r)$ and narrow $(b-a<r)$ shells in the case of ellipsoidal shells $E_{a, b}$.

Given a quadratic form $Q$ of Diophantine type $(\kappa, A)$, i.e. $Q$ satisfies (1.12), we shall apply Corollary 4.11 in order to estimate the Diophantine factors explicitly. Hereby, we prove quantitative bounds in the Oppenheim conjecture (for indefinite quadratic forms $Q$ of Diophantine type $(\kappa, A)$ ) by comparing the volume with the corresponding lattice sum.

Proof of Corollary 1.7 We begin by applying Corollary 7.11 with $b=-a=$ $\varepsilon$ and $\beta=2 / d+\delta^{\prime} / d$ for an appropriate $\delta^{\prime}>0$ : Taking $T_{-} \asymp_{\beta, d}$ $q^{-1 /(d(1 / 2-\beta))}|\operatorname{det} Q|^{-1 / d}$, so that $b_{\beta, d}(b-a) d_{Q} r^{d-2} a_{Q} c_{Q} T_{-}^{d(1 / 2-\beta)} \leq$ (vol $H_{r}$ ) $/ 5$ holds, yields the lattice remainder bound

$$
\Delta_{r} \leq \frac{2 \operatorname{vol} H_{r}}{5}+r^{d-2} C_{Q} b_{\beta, d}\left(2 \varepsilon \gamma_{\left[T_{-}, 1\right], \beta}(r)+\gamma_{\left(1, T_{+}\right], \beta}(r) \log \left(2 \varepsilon T_{+}\right)\right) .
$$

This estimate is valid provided that $r \gg_{\beta, d}\left(q / q_{0}\right)^{(d+1) /(d-2)} q^{1 / 2+2 /(d-4)+\delta}$. Note that we have $T_{-} \in\left[q_{0}^{-1 / 2} r^{-1}, 1\right]$ as required and that the assumptions of Corollary 7.11 are satisfied as well. Next we calibrate the parameter $T_{+}$by taking

$$
T_{+} \asymp_{\beta, d} \varepsilon^{-1} \max \left\{1, \log \left(2 \varepsilon\left(q c_{\beta, d}\right)^{-1}\right)^{2}\right\} .
$$

Since $Q$ is of Diophantine type $(\kappa, A)$, we can use Corollary 4.11 in order to find that

$$
\gamma_{\left[T_{-}, 1\right], \beta}(r) \ll Q, \beta, d A^{-\frac{1-2 \beta}{2(\kappa+1)}} r^{-\frac{1-2 \beta}{\kappa+1}}
$$

and also that

$$
\gamma_{\left(1, T_{+}\right], \beta}(r) \ll Q, \beta, d A^{-\frac{1-2 \beta}{2(\kappa+1)}} r^{-\frac{1-2 \beta}{\kappa+1}}\left(\varepsilon^{-1} \log \left(\varepsilon^{-1}\right)\right)^{\frac{\kappa}{\kappa+1}\left(\frac{1}{2}-\beta\right)} .
$$

In view of (7.12), we may increase $r \gg_{Q, \beta, d} \max \left\{A^{-1}, 1\right\}$ to get

$$
2 b_{\beta, d} C_{Q} r^{d-2} \gamma_{\left[T_{-}, 1\right], \beta}(r) \leq\left(\operatorname{vol} H_{r}\right) / 5 .
$$

Now, we choose $r \asymp_{A, Q, \delta, d} \varepsilon^{-(2 d+3 \kappa d-4 \kappa) /(2 d-8)-\delta}$ in order to obtain

$$
b_{\beta, d} C_{Q} r^{d-2} \log \left(2 \varepsilon T_{+}\right) \gamma_{\left(1, T_{+}\right], \beta}(r) \leq\left(\operatorname{vol} H_{r}\right) / 5 .
$$

All in all, we have

$$
5 \operatorname{vol}_{\mathbb{Z}} H_{r} \geq \operatorname{vol} H_{r} \gg_{d} d_{Q} \varepsilon r^{d-2} .
$$


Since $(2 d+3 \kappa d-4 \kappa) /(2 d-8) \geq 1 /(d-2)$ holds if $d \geq 5$, we find that $\operatorname{vol}_{\mathbb{Z}} H_{r}>1$. This means that there exists at least one non-zero lattice point $m \in \mathbb{Z}^{d}$ satisfying both $|Q[m]|<\varepsilon$ and also $\left\|Q_{+}^{1 / 2} m\right\| \ll_{d} r$.

We can argue similarly to investigate the density of values of a quadratic form:

Proof of Corollary 1.8 It is sufficient to prove that $\operatorname{vol}_{\mathbb{Z}^{d}}\left(r \Omega \cap E_{a, b}\right)>0$ for any $\max \{|a|,|b|\} \leq c_{0} r^{2} / 2$, where $c_{0}$ is as in Lemma 7.1, with $r^{-\nu_{0}+\delta}=b-a$ for $r \geq c_{\delta, d, \Omega, Q, A, \kappa}$ and a sufficiently large constant $c_{\delta, d, \Omega, Q, A, \kappa}>1$. In particular, we consider small shells, i.e. $b-a \leq 1$. Repeating the proof of Corollary 7.11, we see that Corollary 7.11 is also valid for arbitrary parallelepipeds satisfying (7.1), but then the constants depend additionally on the scaling parameter $c_{B} \geq 1$. Also repeating the previous proof (of Corollary 1.7) in this situation shows that we can take $r=c_{\delta, d, \Omega, Q, A, \kappa}(b-a)^{-1 / v_{0}}$, where $v_{0}:=\frac{2(d-4)}{2 d+3 \kappa d-4 \kappa}$, to ensure that $\operatorname{vol}_{\mathbb{Z}^{d}}\left(r \Omega \cap E_{a, b}\right)>0$.

Using the Diophantine estimates for quadratic forms $Q$ of Diophantine type $(\kappa, A)$, we can estimate $\rho_{Q, b-a}^{\text {hyp }+}(r)$ and $\rho_{Q, b-a}^{\text {hyp }-}(r)$ in Corollary 2.5 explicitly as follows.

Proof of Corollary 2.6 First, we consider 'wide shells', i.e. $b-a \geq q$. By applying Corollary 4.11, we can bound the Diophantine factor from Corollary 2.5 by

$$
\begin{array}{r}
\rho_{Q, b-a}^{\text {hyp }}(r) \ll_{d} \inf _{T_{-}, T_{+}}^{*}\left\{\operatorname { l o g } ( ( b - a ) T _ { - } ^ { - ( \frac { d - 4 } { 2 } - \delta ) } + 1 ) ^ { d } \left(q \left(q^{\frac{3}{2}+\delta}\left(a_{Q}^{2} T_{-}^{\frac{d-4}{2}-\delta}+q^{v} A^{-v} T_{-}^{-v} r^{-2 v}\right)\right.\right.\right. \\
\left.\left.\left.+q^{v} A^{-v} T_{+}^{\kappa v} r^{-2 v} \log \left(T_{+}+1\right)\right)+c_{Q} \frac{\log \left(q T_{+}+1\right)}{T_{+}}\right)\right\},
\end{array}
$$

where $v:=(1-2 \beta) /(2 \kappa+2)$ and the infimum is taken over all $T_{-} \in$ $\left[q_{0}^{-1 / 2} r^{-1}, 1\right]$ and $T_{+} \geq 1$. Next we optimize this expression by taking $T_{-}=$ $r^{-2 v /(v+\sigma)}$ and $T_{+}=r^{(2 v) /(\kappa v+1)}$, where $\sigma:=d(1 / 2-\beta)$ : This parameter choice is permissible, since $T_{-} \in\left[q_{0}^{-1 / 2} r^{-1}, 1\right]$ holds (because of $\sigma \geq v$ ), and thus we obtain

$$
\rho_{Q, b-a}^{\text {hyp }+}(r) \ll_{\beta, d} \log (r+1)^{d} h_{Q} q^{\frac{3}{2}+\delta+v}\left(1+A^{-\nu}\right)\left(r^{-\frac{2 v \sigma}{v+\sigma}}+r^{-\frac{2 v}{k v+1}} \log (q r+1)\right),
$$

where $h_{Q}:=q|\operatorname{det} Q|^{1 / 2-\beta}$ (here we avoided to give an optimal estimate in terms of $|\operatorname{det} Q|$ to reduce the notational burden). In view of the bound from Corollary 2.5 and (7.12) we get the relative lattice error

$$
\begin{aligned}
\left|\frac{\operatorname{vol}_{\mathbb{Z}} H_{r}}{\operatorname{vol} H_{r}}-1\right| \ll Q, \Omega, \beta, d(b-a)^{-1} \log (r+1)^{d}\left(r^{-\frac{2 v \sigma}{v+\sigma}}\right. & +r^{-\frac{2 v}{k v+1}} \log (r+1) \\
& \left.+r^{-\frac{d}{2}+2} \log \left(1+\frac{b-a}{r}\right)\right) .
\end{aligned}
$$


For 'thin shells', i.e. $b-a \leq q$, we have

$$
\begin{array}{r}
\rho_{Q, b-a}^{\text {hyp }-}(r) \ll_{\beta, d} \inf _{T_{-}, T_{+}}^{*}\left\{h _ { Q } \operatorname { l o g } ( 1 + T _ { - } ^ { - } { } ^ { - \frac { d - 4 } { 2 } + \delta ) } ) ^ { d } \left((b-a)\left(T_{-}^{\frac{d}{2}-2-\delta}+q^{v} A^{-v} T_{-}^{-v} r^{-2 v}\right)\right.\right. \\
\left.\left.\left.+q^{v} A^{-v} T_{+}^{\kappa v} r^{-2 v}\left(\log \left((b-a)^{*} T_{+}\right)\right)+1\right)\right)\right\},
\end{array}
$$

where the infimum is taken over all $T_{-} \in\left[r^{-1}, 1\right]$ and $T_{+} \geq 1$ satisfying

$$
T_{+} \geq 4(b-a)^{-1} T_{-}^{-\left(\frac{d}{2}-2-\delta\right)} \max \left\{1, \log \left(c_{Q}^{2}(b-a) T_{-}^{-\left(\frac{d}{2}-2-\delta\right)}\right)^{2}\right\} .
$$

\section{Small values of quadratic forms at integer points}

Finally we shall prove Theorem 1.3 by using our effective equidistribution results (in form of Corollary 7.11) together with bounds on small zeros of indefinite integral quadratic forms. Our proof is based on the following strategy: If $Q$ has 'good' Diophantine properties, we can compare the volume with the number of lattice points to establish bounds for non-trivial lattice points $m \in \mathbb{Z}^{d} \backslash\{0\}$ satisfying the Diophantine inequality $|Q[m]|<\varepsilon$. Otherwise $Q$ is near a rational form and here we shall use Schlickewei's bound [51] for small zeros of integral quadratic forms.

\subsection{Integer-valued quadratic forms}

In this section we summarize some essential results on small zeros of integervalued quadratic forms. Here $A[m]$ denotes an integer-valued indefinite quadratic form on a lattice $\Lambda$ in $\mathbb{R}^{d}$ of full rank. Meyer [44] proved in 1884 that such a form represents zero non-trivially on $\Lambda$ if $d \geq 5$. Nowadays, this result is usually deduced from the Hasse-Minkowski theorem, which is a local-global principle (see [25], Theorem 5.7, Corollary 5.10).

Similarly to the result of Birch and Davenport [3] on diagonal forms in five variables, our quantitative bounds in Theorem 1.3 depend essentially on explicit bounds for small zeros of integral forms (see Corollary 8.4). First bounds of this kind were proved by Cassels [12], based on a geometric argument. Birch and Davenport improved Cassels' result as follows: If $d \geq 3$ and $A[m]$ admits a non-trivial zero on the lattice $\Lambda$, then there exists an isotropic lattice point $m \in \Lambda \backslash\{0\}$ with Euclidean norm

$$
0<\|m\|^{2} \leq \gamma_{d-1}^{d-1}\left(2 \operatorname{Tr} A^{2}\right)^{(d-1) / 2}(\operatorname{det} \Lambda)^{2},
$$

where $\gamma_{d}$ denotes the Hermite constant in dimension $d$ (see $[4,16]$ ). This bound is essentially best possible in view of an example by M. Kneser, see [13], if $A$ has signature $(d-1,1)$. In 1985 Schlickewei [51] extended Cassels' 
argument non-trivially by showing that the dimension, say $d_{0}$, of a maximal rational isotropic subspace has an essential impact on the size of small zeros, rather than mere indefiniteness (i.e. $d_{0} \geq 1$ ). He established the following relation between small zeros of integral forms and the dimension $d_{0}$.

Theorem 8.1 (Schlickewei [51]) Let $\Lambda$ be a d-dimensional lattice and A a non-trivial quadratic form in $d$ variables taking integral values on $\Lambda$. Also let $d_{0} \geq 1$ be maximal such that there exists a $d_{0}$-dimensional sublattice of $\Lambda$ on which A vanishes. Then there exist linearly independent lattice points $m_{1}, \ldots, m_{d_{0}} \in \Lambda$, spanning an isotropic subspace, of size

$$
\left(\left\|m_{1}\right\| \ldots\left\|m_{d_{0}}\right\|\right)^{2} \ll_{d}\left(\operatorname{Tr} A^{2}\right)^{\left(d-d_{0}\right) / 2}(\operatorname{det} \Lambda)^{2} .
$$

In the same way as Birch and Davenport [4] deduce their Theorem B from their Theorem A, we may conclude

Theorem 8.2 (Schlickewei [51]) Let $F, G \neq 0$ be quadratic forms in $d$ variables and suppose in addition that $G$ is positive definite. Let $d_{0}$ be maximal such that $F$ vanishes on a rational subspace of dimension $d_{0}$. Then there exist $d_{0}$ linearly independent lattice points $m_{1}, \ldots, m_{d_{0}} \in \mathbb{Z}^{d}$ such that $F$ vanishes on the corresponding subspace and

$$
G\left[m_{1}\right] \cdots G\left[m_{d_{0}}\right] \ll_{d}\left(\operatorname{Tr}\left(F G^{-1}\right)^{2}\right)^{\left(d-d_{0}\right) / 2} \operatorname{det} G,
$$

where the implicit constant depends on d only.

Using an induction argument combined with Meyer's theorem, Schlickewei derived also the following lower bound (8.3) - which we only state for nonsingular forms - for the dimension of a maximal rational isotropic subspace in terms of the signature $(r, s)$. For notational convenience, we may suppose that $r \geq s$. Then Hilfsatz of Section 4 in [51] reads

$$
d_{0} \geq \begin{cases}s & \text { if } r \geq s+3 \\ s-1 & \text { if } r=s+2 \text { or } r=s+1 \\ s-2 & \text { if } r=s\end{cases}
$$

Remark 8.3 One can complement Schlickewei's lower bound (8.3) with the upper bound $d_{0} \leq \min \{r, s\}$, which follows immediately by a dimension argument: If we decompose $\mathbb{R}^{d}=V_{+} \oplus V_{-}$into subspaces $V_{+}, V_{-}$, on which $Q$ is positive or negative definite, and if $V_{\text {iso }}$ denotes an isotropic subspace, then $V_{\text {iso }} \cap V_{ \pm}=\{0\}$ and thus

$$
\operatorname{dim}\left(V_{\text {iso }}\right)=\operatorname{dim}\left(V_{\text {iso }}+V_{ \pm}\right)-\operatorname{dim}\left(V_{ \pm}\right) \leq d-\operatorname{dim}\left(V_{ \pm}\right) .
$$


In particular, the lower bound (8.3) is essentially optimal.

Obviously, a straightforward combination of the upper bound (8.3) together with Theorem 8.1 yields explicit bounds on the smallest non-trivial isotropic vector. However this application can be improved in the cases $r=s+2$ and $r=s$ by reducing the problem to dimension $d-1$ as done by Schlickewei in Folgerung 3 of [51], were he proved that for any integral quadratic form $A$ of signature $(r, s)$ there exists an isotropic lattice point $m \in \mathbb{Z}^{d} \backslash\{0\}$ such that $\|m\|^{2} \ll_{d}\left(\operatorname{Tr} A^{2}\right)^{\rho}$, where

$$
\rho:=\rho(r, s):= \begin{cases}\frac{1}{2} \frac{r}{s} & \text { for } r \geq s+3 \\ \frac{1}{2} \frac{s+2}{s-1} & \text { for } r=s+2 \text { or } r=s+1 \\ \frac{1}{2} \frac{s+1}{s-2} & \text { for } r=s\end{cases}
$$

as defined in (1.10) (see Sect. 1.2). We shall extend this result to general lattices leading to the following strengthening of (8.1).

Corollary 8.4 Suppose that A is a non-singular quadratic form of signature $(r, s)$ in $r+s=d \geq 5$ variables, which takes integral values on $\Lambda$. Additionally suppose that $|\operatorname{det}(\Lambda)| \geq 1$, then the smallest non-trivial isotropic vector $m \in \Lambda$ of A satisfies

$$
0<\|m\|^{2} \ll_{d} \max \left\{\left(\operatorname{Tr} A^{2}\right)^{\frac{1}{2}},\left(\operatorname{Tr} A^{2}\right)^{\rho}\right\}|\operatorname{det} \Lambda|^{\frac{4 \rho+2}{d}}
$$

where $\rho$ is as defined in (1.10).

Compared to (8.1), the exponent in (8.4) is considerably smaller for a wide range of signatures $(r, s)$. Especially, if $r \sim s$, then $\rho \sim 1 / 2$ and therefore $(2 \rho+1) / d \sim 2 / d$.

Proof As can be checked easily, in the cases $r \geq s+3$ and $r=s+1$ the bound (8.4) follows immediately from Theorem 8.1 together with (8.3), since $d / d_{0} \leq 2 \rho+1$ and $2 \leq d / d_{0}$ (by Remark 8.3) in both cases. (Here we estimate $\left(\operatorname{Tr} A^{2}\right)^{\left(d-d_{0}\right) / 2}$ by $\left(\operatorname{Tr} A^{2}\right)^{1 / 2}$ if $\operatorname{Tr} A^{2}<1$ and by $\left(\operatorname{Tr} A^{2}\right)^{\rho}$ if $\operatorname{Tr} A^{2} \geq 1$.) If $r=s$ or $r=s+2$, then the first relation does not hold. Here we fix a reduced basis $v_{1}, \ldots, v_{d}$ of $\Lambda$ with

$$
\left\|v_{1}\right\| \leq \ldots \leq\left\|v_{d}\right\| \text { and }|\operatorname{det}(\Lambda)| \asymp_{d}\left\|v_{1}\right\| \ldots\left\|v_{d}\right\| .
$$

Let $\Lambda_{0}:=\mathbb{Z} v_{1}+\ldots+\mathbb{Z} v_{d-1}$, which is a $d-1$ dimensional sublattice of $\Lambda$, and note that Hadamard's inequality shows that $\operatorname{det}\left(\Lambda_{0}\right)=\left\|v_{1} \wedge \ldots \wedge v_{d-1}\right\| \leq$ $\left\|v_{1}\right\| \ldots\left\|v_{d-1}\right\|$. Thus

$$
\operatorname{det}\left(\Lambda_{0}\right) \ll_{d} \operatorname{det}(\Lambda)^{(d-1) / d}
$$


Now denote by $A_{0}$ the restriction of $A$ to the subspace generated by $v_{1}, \ldots, v_{d-1}$. It follows that $A_{0}$ has signature either $(r, s-1)$ or $(r-1, s)$ and, since $\left(\operatorname{Tr} A^{2}\right)^{1 / 2}=\|A\|_{\mathrm{HS}}$, also that $\operatorname{Tr} A_{0}^{2} \leq \operatorname{Tr} A^{2}$. Applying Theorem 8.1 (resp. Theorem 8.2 after a coordinate change) to $A_{0}$ and $\Lambda_{0}$ shows that there exists an isotropic lattice point $m \in \Lambda_{0} \backslash\{0\}$ such that

$$
\|m\|^{2} \ll_{d}\left(\operatorname{Tr} A_{0}^{2}\right)^{\frac{d-1-d_{0}}{2 d_{0}}}\left|\operatorname{det} \Lambda_{0}\right|^{\frac{2}{d_{0}}} \ll_{d}\left(\operatorname{Tr} A^{2}\right)^{\frac{d-1-d_{0}}{2 d_{0}}}|\operatorname{det} \Lambda|^{\frac{d-1}{d} \frac{2}{d_{0}}},
$$

where $d_{0}$ denotes the dimension of a maximal isotropic subspace of $A_{0}$ (instead of $A$ ). Completing the proof, we note that in both cases $r=s+2$ and $r=s$ one has

$$
2 \leq(d-1) / d_{0} \leq 2 \rho+1,
$$

as can be readily seen.

Remark 8.5 In 1988 Schlickewei and Schmidt [56] complemented their work [55] on isotropic subspaces of quadratic forms showing that Schlickewei's bound in terms of $d_{0}$ is best possible. Additionally, one can also ask if Schlickewei's bound (8.3) in terms of $(r, s)$ is best possible, as was already conjectured by Schlickewei himself in [51]. At least for the cases $r \geq s+3$ and $(3,2)$ this is known and due to Schmidt, see [49].

Remark 8.6 As a final remark we note that in the Geometry of Numbers it is often the case that one can use the existence of a lattice points satisfying some inequality in order to get several independent points satisfying a joint inequality. This argument was used by Schlickewei and Schmidt $[55,57]$ to prove an extension of Theorem 8.1, in which they considered several isotropic subspaces and their relative position.

\subsection{Proof of Theorem 1.3}

Now we are in position to prove the second main theorem of this paper. To simplify the notation we may replace $Q$ by $Q / \varepsilon$ and consider the solubility of the Diophantine inequality $|Q[\mathrm{~m}]|<1$. Notice that this rescaling does not change the constant $c_{B}=1$ occuring in Corollary 7.11.

Proof of Theorem 1.3 Let $d \geq 5, q_{0} \geq 1$ and

$$
r \geq \tilde{b}_{\beta, d} q^{1 / 2}\left(q / q_{0}\right)^{(d+1) /(d-2)}
$$

as in Corollary 7.11 and $\beta=2 / d+\delta^{\prime} / d$ with fixed $\delta^{\prime}>0$ depending on $\delta>0$. Applying Corollary 7.11 with $b=-a=1 / 5$ (note that both conditions 
$\max \{|a|,|b|\} \leq r^{2} / 5$ and $b-a \leq 1$ are satisfied) gives the bound

$$
\Delta_{r} \leq \frac{\operatorname{vol~} H_{r}}{5}+b_{\beta, d} d_{Q} r^{d-2} q c_{Q}\left(c_{Q} T_{-}^{d(1 / 2-\beta)}+\gamma_{\left[T_{-}, 1\right], \beta}(r)+\gamma_{\left(1, T_{+}\right], \beta}(r) \log \left(T_{+}\right)\right)
$$

for any $T_{-} \in\left[q_{0}^{-1 / 2} r^{-1}, 1\right]$ and

$$
\left.T_{+} \gg_{\beta, d} \max \left\{1, \log \left(10 c_{\beta, d} q\right)\right)^{2}\right\} \gg_{\beta, d} \log (q+1)^{2} .
$$

Hence, we can take $T_{+} \asymp \beta, d \log (q+1)^{2}$. Additionally, by taking

$$
T_{-} \asymp_{\beta, d} q^{-2 /(d-4)-\delta / 4}|\operatorname{det} Q|^{-1 / d}
$$

we can also ensure that

$$
b_{\beta, d} d_{Q} r^{d-2} q|\operatorname{det} Q|^{1 / 2-\beta} T_{-}^{d(1 / 2-\beta)} \leq\left(\operatorname{vol} H_{r}\right) / 10,
$$

compare the lower bound (7.12) of Lemma 7.1. At this step we have to choose

$$
r \gg_{\beta, d}\left(q / q_{0}\right)^{1 / 2} q^{1 / 2+2 /(d-4)+\delta / 4} \geq q_{0}^{-1 / 2}|\operatorname{det} Q|^{1 / d} q^{2 /(d-4)+\delta / 4}
$$

in order to guarantee that $T_{-} \in\left[q_{0}^{-1 / 2} r^{-1}, 1\right]$ is satisfied.

First Case: We consider first classes of quadratic forms $Q$ for which the lattice remainder is 'small': Corresponding to Diophantine properties of $Q$, we assume that

$$
\begin{array}{r}
b_{\beta, d} q|\operatorname{det} Q|^{1 / 4-\beta / 2} \gamma_{\left[T_{-}, 1\right], \beta}(r) \leq h_{\beta, d} \text { and } \\
b_{\beta, d} q|\operatorname{det} Q|^{1 / 4-\beta / 2} \gamma_{\left[1, T_{+}\right], \beta}(r) \log \left(T_{+}\right) \leq h_{\beta, d}
\end{array}
$$

with some constant $h_{\beta, d}>0$ depending on $d$ and $\beta$ only (compare again with (7.12)) such that $5 \operatorname{vol}_{\mathbb{Z}} H_{r} \geq \operatorname{vol} H_{r}$. Note that $r \geq q^{1 / 2}$ is fixed here. According to Corollary 7.11 and (8.6) we shall take a priori

$$
r \asymp_{\beta, d}\left(q / q_{0}\right)^{(d+1) /(d-2)} q^{1 / 2+2 /(d-4)+\delta} .
$$

Increasing the implict constant guarantees that $\operatorname{vol}_{\mathbb{Z}} H_{r} \geq 2$, i.e. there exists at least one non-zero lattice point $m \in \mathbb{Z}^{d} \backslash\{0\}$ satisfying both $|Q[m]| \leq 1$ and $\left\|Q_{+}^{1 / 2} m\right\| \leq r$. Because of $\rho \geq 1 / 2$, it is easy to see that the right-hand side of (8.8) is bounded, up to absolute constants, by the right-hand side of (1.9).

Second Case: Now we assume that one of the inequalities in (8.7) fails. Then 
there exists a $t_{0} \in\left[T_{-}, T_{+}\right]$such that the reciprocal $\alpha_{d}$-characteristic satisfies at least

$$
\beta_{t_{0} ; r}^{-1}=d_{Q} r^{d} \alpha_{d}\left(\Lambda_{t_{0}}\right)^{-1} \ll \beta, d E\left(t_{0}\right) \stackrel{\text { def }}{=}(q \log \log (q+\exp (1)))^{\frac{2 d}{d-4}+\delta / 4}
$$

Following the proof of Lemma 4.10, we see that there exists a $d$-dimensional sublattice $\Lambda^{\prime} \subset \Lambda_{t_{0}}$ with $\alpha_{d}\left(\Lambda_{t_{0}}\right)=\left|\operatorname{det} \Lambda^{\prime}\right|^{-1}=\left\|w_{1} \wedge \ldots \wedge w_{n}\right\|^{-1}$, where

$$
w_{j}=\left(\begin{array}{c}
r Q_{+}^{-1 / 2}\left(m_{j}-4 t_{0} Q n_{j}\right) \\
r^{-1} Q_{+}^{1 / 2} n_{j}
\end{array}\right)
$$

is a basis of $\Lambda^{\prime}$ determined by integral vectors $m_{j}, n_{j} \in \mathbb{Z}^{d}, j=1, \ldots, d$. We have also proven, writing $N=\left(n_{1}, \ldots, n_{d}\right), M=\left(m_{1}, \ldots, m_{d}\right) \in \mathrm{M}(d, \mathbb{Z})$, that $N$ is invertible with $\beta_{t_{0} ; r}^{-1}>|\operatorname{det} N|$ and that the estimate

$$
\alpha_{d}\left(\Lambda_{t_{0}}\right)^{-1} \gg_{d} r^{-(d-2)} q^{-1}|\operatorname{det} Q|^{1 / 2}|\operatorname{det} N|\left\|M N^{-1}-4 t_{0} Q\right\|
$$

holds, provided that $\alpha_{d}\left(\Lambda_{t_{0}}\right)>q d_{Q} r^{d-2}$. In view of (8.9) the last condition is satisfied if we take a priori

$$
r \gg_{\beta, d}\left(E\left(t_{0}\right) q\right)^{1 / 2} .
$$

Now we are in position to apply Corollary 8.4 with the rescaled lattice $\Lambda=r \Lambda^{\prime}$, noting that $\operatorname{det}(\Lambda)=r^{d} \operatorname{det}\left(\Lambda^{\prime}\right) \geq|\operatorname{det} Q|^{1 / 2}|\operatorname{det} N| \geq 1$, and the quadratic form $A[x]=\langle x, A x\rangle$ induced by the symmetric matrix

$$
A \stackrel{\text { def }}{=}\left(\begin{array}{cc}
0 & r^{-2} \mathbb{1}_{d} \\
r^{-2} \mathbb{1}_{d} & 8 t_{0} S
\end{array}\right)
$$

with $\left\langle w_{i}, A w_{j}\right\rangle=\left\langle m_{i}, n_{j}\right\rangle+\left\langle m_{j}, n_{i}\right\rangle$. In other words, the quadratic form $A$ is represented by the symmetric matrix $A_{0}:=N^{T} M+M^{T} N$ in coordinates $w_{1}, \ldots, w_{d}$. In particular, $A$ is integer-valued on $\Lambda$. Since $A_{1}[n]:=$ $A_{0}\left[N^{-1} n\right]$, i.e. $A_{1}=M N^{-1}+\left(M N^{-1}\right)^{T}$, has the same signature as $A_{0}$, we need to check that the signature of $A_{1}$ is $(r, s)$. Because of

$$
\left\|A_{1}-8 t_{0} Q\right\| \ll_{\beta, d}|\operatorname{det} N|^{-1} r^{-2} q E\left(t_{0}\right)
$$

we may choose a priori $r \gg_{\beta, d}\left(q / q_{0}\right)^{1 / 2} \max \left\{1, t_{0}^{-1 / 2}\right\} q^{d /(d-4)+\delta}$, i.e.

$$
r \gg_{\beta, d}\left(q / q_{0}\right)^{1 / 2} q^{1 / 2+(d+1) /(d-4)+\delta}
$$


to ensure that $A_{1}$ and $t_{0} Q$ have the same number of eigenvalues with the same sign, i.e. the same signature (e.g. apply the Hoffman-Wielandt inequality, see Theorem 6.3.5 in [30]). Thus, there exists a non-trivial lattice point $w=$ $a_{1} r w_{1}+\ldots+a_{d} r w_{d} \in \Lambda$, where $\left(a_{1}, \ldots, a_{d}\right) \in \mathbb{Z}^{d} \backslash\{0\}$, which satisfies $A[w]=0$ and, writing $n_{0}=a_{1} n_{1}+\ldots+a_{d} n_{d} \in \mathbb{Z}^{d} \backslash\{0\}$, is of size

$$
\begin{aligned}
\left\|Q_{+}^{1 / 2} n_{0}\right\|^{2} \leq\|w\|^{2} & \ll_{d} \max \left\{\left(\operatorname{Tr} A^{2}\right)^{\frac{1}{2}},\left(\operatorname{Tr} A^{2}\right)^{\rho}\right\}|\operatorname{det} \Lambda|^{\frac{4 \rho+2}{d}} \\
& \ll_{\beta, d} \log (q+1)^{4 \rho}\left(|\operatorname{det} Q|^{1 / 2} E\left(t_{0}\right)\right)^{\frac{4 \rho+2}{d}} \\
& \ll_{\beta, d} q^{\delta+\frac{8 \rho+4}{d-4}}|\operatorname{det} Q|^{\frac{2 \rho+1}{d}}
\end{aligned}
$$

where we used $\operatorname{Tr} A^{2} \ll_{d}\left(r^{-2}+t_{0}\right)^{2} \ll t_{0}^{2} \ll_{\beta, d} \log (q+1)^{4}$ and (8.9). Writing $w=\left(w_{1}, w_{2}\right) \in \mathbb{R}^{d} \times \mathbb{R}^{d}$ we also see that $0=A[w]=r^{-2}\left\langle w_{1}, w_{2}\right\rangle+$ $8 t_{0} Q\left[n_{0}\right]$ and thus

$$
\begin{aligned}
\left|Q\left[n_{0}\right]\right| & \ll\left(r^{2} t_{0}\right)^{-1}\left\|w_{1}\right\| \cdot\left\|w_{2}\right\| \leq\left(r^{2} t_{0}\right)^{-1}\|w\|^{2} \\
& \ll_{d} \max \left\{1, t_{0}^{2 \rho-1}\right\}|\operatorname{det} \Lambda|^{\frac{4 \rho+2}{d}} r^{-2} \ll_{\beta, d} q^{\delta+\frac{8 \rho+4}{d-4}}|\operatorname{det} Q|^{\frac{2 \rho+1}{d}} r^{-2} .
\end{aligned}
$$

Hence, requiring in addition

$$
r \gg_{\beta, d} q^{\frac{1}{2}+\frac{d \rho+2}{d-4}+\delta} \geq q^{\delta+\frac{4 \rho+2}{d-4}}|\operatorname{det} Q|^{\frac{2 \rho+1}{2 d}},
$$

it follows from (8.13) that $\left|Q\left[n_{0}\right]\right| \ll_{\beta, d} 1$, which in turn guarantees $\left|Q\left[n_{0}\right]\right|<$ 1 as long as $r$ is taken large enough in terms of $\beta$ and $d$. Combining this choice with the lower bounds on $r$ already required in (8.5), (8.6), (8.10) (8.11) and (8.14), we observe that an appropriate choice for $r$ is given by

$$
r \asymp \beta, d\left(q / q_{0}\right)^{\frac{d+1}{d-2}} q^{\frac{1}{2}+\frac{\max \{\rho d+2, d+1\}}{d-4}+\delta},
$$

where the implicit constant is chosen large enough depending on $\beta$ and $d$ only. This concludes the proof of Theorem 1.3.

Funding Open Access funding enabled and organized by Projekt DEAL.

Open Access This article is licensed under a Creative Commons Attribution 4.0 International License, which permits use, sharing, adaptation, distribution and reproduction in any medium or format, as long as you give appropriate credit to the original author(s) and the source, provide a link to the Creative Commons licence, and indicate if changes were made. The images or other third party material in this article are included in the article's Creative Commons licence, unless indicated otherwise in a credit line to the material. If material is not included in the article's Creative Commons licence and your intended use is not permitted by statutory regulation or exceeds the permitted use, you will need to obtain permission directly from the copyright holder. To view a copy of this licence, visit http://creativecommons.org/licenses/by/4.0/. 


\section{Index}

$(b-a)^{*} \stackrel{\text { def }}{=}(b-a) I(b-a \leq 1)+I(b-a>1) 871,914$

$(b-a)_{q}:=(b-a) I(b-a \leq q)+q^{(2 \beta d-1) / 2} I(b-a>q) 871,913$

$\langle\cdot, \cdot\rangle,\|\cdot\|$, Euclidean inner product and associated norm 861, 870

$\phi_{r}(x)=\phi(x / r)$, for a function $\phi$ on $\mathbb{R}^{m}, 875$

$A \ll_{B} C, A \asymp_{B} C$, Vinogradov's notation 868,871

$f^{*}$, radial realization of a bi-K-invariant function on $\mathrm{G}, 897$

$\alpha, \alpha_{l}$-characteristic of a lattice, 870,884

$A_{g}$, mean-value operator on $\mathrm{G}, 897$

$a_{Q}:=q c_{Q}, 927$

$\beta$, exponent in the range $\left(\frac{2}{d}, \frac{1}{2}\right), 870$

$\beta_{t ; r}:=\alpha_{d}\left(\Lambda_{t}\right) r^{-d}|\operatorname{det} Q|^{1 / 2}, 891$

$c_{Q}:=|\operatorname{det} Q|^{1 / 4-\beta / 2}, 871$

$C_{Q}:=q|\operatorname{det} Q|^{-1 / 4-\beta / 2}, 871$

Cartan decomposition of $g \in \operatorname{SL}(2, \mathbb{R}): g=k_{1}(g) d(g) k_{2}(g), 896$

$\Delta_{r}:=\left|\operatorname{vol}_{\mathbb{Z}} H_{r}-\operatorname{vol} H_{r}\right|$, lattice point deficiency, 872, 926

$\delta_{t} Q ; R$, rational approximation error of $t Q$ truncated at $R, 890$

$\mathrm{D}:=\left\{d_{r}: r>0\right\}$, diagonal subgroup of $\operatorname{SL}(2, \mathbb{R}), 888$

$d_{\Delta}(L)=d(L)$, covolume of the $\Delta$-rational subspace $L, 883$

$d_{Q}:|\operatorname{det} Q|^{-1 / 2}, 870$

$D_{r Q}$, diagonalizable matrix on $\mathbb{R}^{2 d}, 882,889$

Diophantine quadratic form of type $(\kappa, A) 863$

$E_{a, b}:=\left\{x \in \mathbb{R}^{d}: a<Q[x]<b\right\}$, hyperbolic or ellipsoidal shell, 859, 871

$\gamma_{[a, b], \beta}(r)$, Diophantine factor for $Q$ on $[a, b]$ with exponent $\beta, 870,896$

$\mathrm{G}=\mathrm{SL}(2, \mathbb{R}), 896$

$\widehat{g}_{I}:=\max \left\{\left|\widehat{g}_{w}(t)\right|: t \in I\right\}$, maximum of $\left|\widehat{g}_{w}(t)\right|$ on an interval $I, 896$

$g_{ \pm w}^{Q}(x):=g_{ \pm w}(Q[x]), x \in \mathbb{R}^{d}, 875$

$g_{ \pm w}:=I_{[a, b]_{ \pm w}} * k_{w}, 875$

$\mathcal{H}:=\left\{v \in \Lambda:\|v\|_{\infty}<1\right\}, 883$

$H_{r}:=E_{a, b} \cap r \Omega$, if $Q$ is indefinite, 872

$H_{r}:=E_{0, b}$, with $r=\sqrt{2 b}$, if $Q$ is positive definite, 872

$H_{t}(m, n):=r^{2} Q_{+}^{-1}[m-4 t Q n]+r^{-2} Q_{+}[n], 880$

$\left\|\widehat{I}_{\Omega}\right\|_{1, \varepsilon}:=\int_{\mathbb{R}^{d}}\left|\widehat{I}_{\Omega}(v)\right| \exp \left\{-\|\varepsilon v\|^{1 / 2}\right\} \mathrm{d} v \ll \Omega \varepsilon^{-(d+1) / 2} 919$

$I_{\Delta}:=\left|\int_{J_{0}} R\left(e_{t} Q \mathrm{v}_{r}\right) \widehat{g}_{w}(t) \mathrm{d} t\right| 877$

$I_{\theta}:=\left|\int_{J_{1}} \widehat{g}_{w}(t) \int_{\mathbb{R}^{d}} \theta_{v}(t) \widehat{\zeta}(v) \mathrm{d} v \mathrm{~d} t\right| 877$

$I_{\vartheta}:=\left|\int_{J_{1}} \widehat{g}_{w}(t) \int_{\mathbb{R}^{d}} \vartheta_{v}(t) \widehat{\zeta}(v) \mathrm{d} v \mathrm{~d} t\right| 877$

$I_{B}$, indicator function of a set $B, 864$

Iwasawa decomposition of $g \in \mathrm{SL}(2, \mathbb{R}): g=k(g) t(g), k(g) \in \mathrm{K}$, $t(g) \in \mathrm{T}, 896$

$J_{0}:=\left[-q_{0}^{-1 / 2} r^{-1}, q_{0}^{-1 / 2} r^{-1}\right], 877$ 
$J_{1}:=\mathbb{R} \backslash J_{0}, 877$

$\mathrm{K}:=\mathrm{SO}(2)=\left\{k_{\theta}: \theta \in[0,2 \pi]\right\}$, orthogonal subgroup of $\operatorname{SL}(2, \mathbb{R}), 889$ $k$, compactly supported kernel with sufficiently fast decaying Fourier transform, 864,874

$\Lambda_{Q}$, lattice in $\mathbb{R}^{2 d}$ depending on $Q$ only, 889

$\Lambda_{t}:=D_{r Q} U_{4 t Q} \mathbb{Z}^{2 d}$, lattice on $\mathbb{R}^{2 d}, 882,889$

$\Lambda_{Q, t}:=d_{q^{1 / 2}} u_{4 t} \Lambda_{Q}$, lattice on $\mathbb{R}^{2 d}, 896$

$M_{j}(\Delta), j$-th successive minimum of a lattice $\Delta, 884$

$(\partial \Omega)_{\varepsilon}:=\Omega_{\varepsilon} \backslash \Omega_{-\varepsilon}, \epsilon$-thickened boundary of $\Omega, 915$

$\Omega_{ \pm \varepsilon}:=(1 \pm \varepsilon) \Omega, \epsilon$-thickening resp. thinning of $\Omega, 915$

$\psi(r, t):=\sum_{m, n \in \mathbb{Z}^{d}} \exp \left\{-H_{t}(m, n)\right\}, 880$

$\varphi_{\lambda}: \mathrm{G} \rightarrow \mathbb{R}^{+}$, corresponding to the character $\chi_{\lambda}, 897$

$Q$, as quadratic form and the corresponding symmetric matrix, 861,870

$q$, largest eigenvalue of $Q$ in absolute value, 861, 870

$Q[x]=\langle Q x, x\rangle$, Siegel's notation, 861,870

$Q_{+}$, positive definite square root of $Q^{2}, 861$

$Q_{0}$ signature matrix corresponding to $Q, 889$

$q_{0}$, smallest eigenvalue of $Q$ in absolute value, $q_{0} \geq 1,861,870$

$Q_{r, v}(t, x):=2 \pi \mathrm{i} t Q[x]-r^{-2} Q_{+}[x]+2 \pi \mathrm{i}\left\langle x, v r^{-1}\right\rangle, 877$

$\rho=\rho(r, s)$, Schlickewei exponent, 861, 934

$\rho_{Q}^{\text {ell }}(r), 872,930$

$\rho_{Q, b-a}^{\text {hyp } * *}(r), 929$

$\rho_{Q, b-a}^{\text {hyp } *}(r), 929$

$\rho_{Q, b-a}^{\text {hyp }+}(r), 873,928$

$\rho_{Q, b-a}^{\text {hyp }-}(r), 873,928$

$\rho_{Q, b-a}^{w}(r), 871,874$

$R\left(I_{E_{a, b}} \mathrm{v}_{r}\right), R\left(g \mathrm{v}_{r}\right), R\left(I_{E_{a, b} \cap r \Omega}\right)$, lattice point remainder, 864, 875, 915

$r_{*}:=r q^{-1 / 2} 896$

$r_{t}:=r\left(4 \pi^{2} t^{2} r^{4}+1\right)^{-1 / 2}, 878$

$S$, orthogonal matrix such that $S Q Q_{+}^{-1} S^{T}=Q_{0}, 889$

$s_{[a, b]_{ \pm w}}(t):=\left|(2 \pi t)^{-1} \sin (\pi t(b-a \pm 2 w))\right|, 877$

T, Borel subgroup of $\operatorname{SL}(2, \mathbb{R}), 896$

$\tau_{\lambda}$, spherical function on $\mathrm{G}, 897$

$\theta_{v}(t)$, Theta series for $Q, 865,877$

$\vartheta_{v}(t)$, Theta integral for $Q, 865,877$

$\mathrm{U}:=\left\{u_{t}: t \in \mathbb{R}\right\}$, standard unipotent subgroup of $\mathrm{SL}(2, \mathbb{R}), 888$

$U_{4 t Q}$, unipotent matrix on $\mathbb{R}^{2 d}, 882,889$

$\mathrm{v}$, weight function on $\mathbb{R}^{d}$ of sufficiently fast decay, 870

$\mathrm{v}_{ \pm \varepsilon}:=I_{\Omega_{ \pm \varepsilon}} * k_{B, \varepsilon}, \epsilon$-smoothing of $\Omega, 915$ 
vol $(B)$, Lebesgue measure on $\mathbb{R}^{d}, 859$

$\operatorname{vol}_{\mathbb{Z}}(B)=\#\left(B \cap \mathbb{Z}^{d}\right)$, counting measure on $\mathbb{Z}^{d}, 859$

$\chi_{\lambda}$, character of T, 897

$\|\widehat{\zeta}\|_{*, r}:=q^{d / 4}\left(\left(\frac{q}{q_{0}}\right)^{d / 2}\|\widehat{\zeta}\|_{1}+\int_{\|v\|_{\infty}>r / 2} \frac{|\widehat{\zeta}(v)|}{\left(q^{1 / 2} r^{-1}+\left\|v r^{-1}\right\| \mathbb{Z}\right)^{d / 2}} \mathrm{~d} v\right), 871,880$ $\zeta(x):=\mathrm{v}(x) \exp \left\{Q_{+}[x]\right\}, 875$

\section{References}

1. Athreya, J.S., Margulis, G.A.: Values of random polynomials at integer points. J. Mod. Dyn. 12, 9-16 (2018)

2. Brandolini, L., Colzani, L., Travaglini, G.: Average decay of Fourier transforms and integer points in polyhedra. Ark. Mat. 35(2), 253-275 (1997)

3. Birch, B.J., Davenport, H.: On a theorem of Davenport and Heilbronn. Acta Math. 100, 259-279 (1958)

4. Birch, B.J., Davenport, H.: Quadratic equations in several variables. Proc. Camb. Philos. Soc. 54, 135-138 (1958)

5. Bentkus, V., Götze, F.: On the lattice point problem for ellipsoids. Acta Arith. 80(2), 101125 (1997)

6. Bentkus, V., Götze, F.: Lattice point problems and distribution of values of quadratic forms. Ann. Math. (2) 150(3), 977-1027 (1999)

7. Buterus, P., Götze, F., Hille, T.: On small values of indefinite diagonal quadratic forms at integer points in at least five variables. Trans. Am. Math. Soc. Ser. B. (2022) (to appear). arXiv: 1810.11898

8. Bochner, S.: Vorlesungen über Fouriersche Integrale. Chelsea Publ. Co., New York (1948)

9. Bourgain, J.: A quantitative Oppenheim theorem for generic diagonal quadratic forms. Isr. J. Math. 215(1), 503-512 (2016)

10. Bhattacharya, R.N., Rao, R.R.: Normal approximation and asymptotic expansions. Reprint of the 1976 original. Robert E. Krieger Publishing Co., Inc., Melbourne (1986)

11. Borevich, A.I., Shafarevich, I.R.: Number theory. Translated from the Russian by Newcomb Greenleaf. Pure and Applied Mathematics, Vol. 20. Academic Press, New York (1966)

12. Cassels, J.W.S.: Bounds for the least solutions of homogeneous quadratic equations. Proc. Camb. Philos. Soc. 51, 262-264 (1955)

13. Cassels, J.W.S.: "Addendum to the paper "Bounds for the least solutions of homogeneous quadratic equations"”. Proc. Camb. Philos. Soc. 52, 602 (1956)

14. Cassels, J.W.S.: An introduction to the geometry of numbers. Classics in Mathematics. Corrected reprint of the 1971 edition. Springer, Berlin (1997)

15. Corless, R.M., Gonnet, G.H., Hare, D.E.G., Jeffrey, D.J., Knuth, D.E.: On the Lambert W function. Adv. Comput. Math. 5(4), 329-359 (1996)

16. Davenport, H.: Note on a theorem of Cassels. Proc. Camb. Philos. Soc. 53, 539-540 (1957)

17. Davenport, H.: Indefinite quadratic forms in many variables. II. Proc. Lond. Math. Soc. (3) 8, 109-126 (1958)

18. Davenport, H., Heilbronn, H.: On indefinite quadratic forms in five variables. J. Lond. Math. Soc. 21, 185-193 (1946)

19. Davenport, H., Lewis, D.J.: Gaps between values of positive definite quadratic forms. Acta Arith. 22, 87-105 (1972)

20. Dani, S.G., Margulis. G.A.: "Limit distributions of orbits of unipotent flows and values of quadratic forms". In: I. M. Gelfand Seminar. Adv. Soviet Math. Amer. Math. Soc., vol. 16, pp. 91-137 (1993)

21. Elsner, G.: Values of special indefinite quadratic forms. Acta Arith. 138(3), 201-237 (2009) 
22. Eskin, A., Margulis, G., Mozes, S.: Quadratic forms of signature $(2 ; 2)$ and eigenvalue spacings on rectangular 2-tori. Ann. Math. (2) 161(2), 679-725 (2005)

23. Eskin, A., Margulis, G., Mozes, S.: Upper bounds and asymptotics in a quantitative version of the Oppenheim conjecture. Ann. Math. (2) 147(1), 93-141 (1998)

24. Einsiedler, M., Ward, T.: Homogeneous Dynamics and Applications (to appear) (2019)

25. Gerstein, L.J.: Basic Quadratic Forms. Graduate Studies in Mathematics, vol. 90. American Mathematical Society, Providence (2008)

26. Ghosh, A., Kelmer, D.: A quantitative Oppenheim theorem for generic ternary quadratic forms. J. Mod. Dyn. 12, 1-8 (2018)

27. Götze, F., Margulis, G.: Distribution of values of quadratic forms at integral points. Preprint (2010). http://arxiv.org/abs/1004.5123v2

28. Götze, F.: Lattice point problems and values of quadratic forms. Invent. Math. 157(1), 195-226 (2004)

29. Helgason, S.: Groups and geometric analysis. Vol. 83. Mathematical Surveys and Monographs. Integral geometry, invariant differential operators, and spherical functions, Corrected reprint of the 1984 original. American Mathematical Society (2000)

30. Horn, R.A., Johnson, C.R.: Matrix Analysis, 2nd edn., p. xviii+643. Cambridge University Press, Cambridge (2013)

31. Ingham, A.E.: A note on Fourier transforms. J. Lond. Math. Soc. 9(1), 29-32 (1934)

32. Knapp, A.W.: Representation theory of semisimple groups. An overview based on examples, Reprint of the 1986 original. Princeton University Press (2001)

33. Knapp, A.W.: Lie Groups Beyond an Introduction. Progress in Mathematics. Birkhäuser, Boston (2002)

34. Kowalski, E.: An Introduction to the Representation Theory of Groups. Graduate Studies in Mathematics, vol. 155. American Mathematical Society, Providence (2014)

35. Korkine, A., Zolotareff, G.: Sur les formes quadratiques positives quaternaires. Math. Ann. 5(4), 581-583 (1872)

36. Korkine, A., Zolotareff, G.: Sur les formes quadratiques. Math. Ann. 6(3), 366-389 (1873)

37. Korkine, A., Zolotareff, G.: Sur les formes quadratiques positives. Math. Ann. 11(2), 242292 (1877)

38. Lang, S.: $\mathrm{SL}_{2}(\mathbf{R})$. Graduate Texts in Mathematics, Vol. 105. Reprint of the 1975 edition. Springer, New York (1985)

39. Lewis, D.J.: The distribution of the values of real quadratic forms at integer points. In: Analytic Number Theory (Proc. Sympos. Pure Math., Vol. XXIV, St. Louis Univ., St. Louis, Mo., 1972). American Mathematical Society, pp. 159- 174 (1973)

40. Margulis, G.A.: Discrete subgroups and ergodic theory. In: Number Theory, Trace Formulas and Discrete Groups (Oslo, 1987), pp. 377-398. Academic Press, Boston (1989)

41. Margulis, G.A.: Oppenheim conjecture. In: Fields Medallists' Lectures. World Sci. Ser. 20th Century Math. (5), pp. 272-327 (1997)

42. Marklof, J.: Pair correlation densities of inhomogeneous quadratic forms II. Duke Math. J. 115(3), 409-434 (2002). (issn: 0012-7094)

43. Marklof, J.: Pair correlation densities of inhomogeneous quadratic forms. Ann. Math. (2) 158(2), 419-471 (2003)

44. Meyer, A.: Ueber die Aufloesung der Gleichung $a x^{2}+b y^{2}+c z^{2}+d u^{2}+e v^{2}$ in ganzen Zahlen. Vierteljahresschrift der Naturforschenden Gesellschaft in Zürich 29, 209-222 (1884)

45. Margulis, G., Mohammadi, A.: Quantitative version of the Oppenheim conjecture for inhomogeneous quadratic forms. Duke Math. J. 158(1), 121-160 (2011)

46. Mumford, D.: Tata lectures on theta. I. Progress in Mathematics, Vol. 28. With the assistance of C. Musili, M. Nori, E. Previato and M. Stillman. Birkhäuser Boston, Inc., Boston (1983)

47. Oppenheim, A.: The minima of indefinite quaternary quadratic forms. Proc. Nat. Acad. Sci. USA 15(9), 724-727 (1929) 
48. Oppenheim, A.: The minima of indefinite quaternary quadratic forms. Ann. Math. (2) 32(2), 271-298 (1931)

49. Schmidt, W.M.: Small zeros of quadratic forms. Trans. Am. Math. Soc. 291(1), 87-102 (1985)

50. Schmidt, W.M.: Asymptotic formulae for point lattices of bounded determinant and subspaces of bounded height. Duke Math. J. 35, 327-339 (1968)

51. Schlickewei, H.P.: Kleine Nullstellen homogener quadratischer Gleichungen. Monatsh. Math. 100(1), 35-45 (1985)

52. Siegel, C.L.: Lectures on the geometry of numbers. Notes by B. Friedman, Rewritten by Komaravolu Chandrasekharan with the assistance of Rudolf Suter, With a preface by Chandrasekharan. Springer, Berlin (1989)

53. Skriganov, M.M.: Constructions of uniform distributions in terms of geometry of numbers. Algebra i Analiz 6(3), 200-230 (1994)

54. Skriganov, M.M.: Ergodic theory on SL(n), Diophantine approximations and anomalies in the lattice point problem. Invent. Math. 132(1), 1-72 (1998)

55. Schlickewei, H.P., Schmidt, W.M.: Quadratic geometry of numbers. Trans. Am. Math. Soc. 301(2), 679-690 (1987)

56. Schlickewei, H.P., Schmidt, W.M.: Quadratic forms which have only large zeros. Monatsh. Math. 105(4), 295-311 (1988)

57. Schlickewei, H.P., Schmidt, W.M.: Isotrope Unterräume rationaler quadratischer Formen. Math. Z. 201(2), 191-208 (1989)

Publisher's Note Springer Nature remains neutral with regard to jurisdictional claims in published maps and institutional affiliations. 